\title{
AUTOMATIC PAGE-TURNER FOR PIANISTS WITH WEARABLE MOTION DETECTOR
}

by

Seyed Ali Mirazimzadeh

A thesis submitted to

the Faculty of Graduate and Postdoctoral Affairs

in partial fulfillment of the requirements for the degree of

MASTER OF APPLIED SCIENCE

Human-computer interaction

at

CARLETON UNIVERSITY

Ottawa, Ontario

November, 2020

(C) Copyright by Seyed Ali Mirazimzadeh, 2020 
و تقديم به يدرم كاوس كه حضور روشنش مرا در اين سفر آرام كرد

Dedicated to my father Kavoos whose ever-present memory glided me through this journey, and to my mother Maliheh whose unwavering well wishes were guiding light. 


\begin{abstract}
Turning book pages is a simple act that readers do while reading. This simple act that we take for granted could be challenging when both hands are engaged in other tasks. In case that a pianist sight-read, using one hand for turning the music sheets can cause distraction or short delay in their performance. Previous studies predominantly utilized audio or video processing techniques for following the musicians' performance. We suggested a framework that uses wearable technologies as an alternative solution for solving page-turning problems. The proposed design recognizes the pianist's wrists oscillatory motion with accelerometer sensors and tries to sync them to digital music notes. This system uses dynamic time warping and cross-correlation methods to find the optimum alignment between actual and captured data and reducing time latency. Eventually, a heuristic decision model assesses the pianist's performance and decides to turn the digital music sheet at predefined points.
\end{abstract}

Keywords: wearable devices, automatic page-turner, time series alignment 


\section{Acknowledgements}

This research started with a long journey, and I think here is the right place to acknowledge people who encouraged me without any expectations.

First of all, I would like to thank my supervisor, Dr. Victoria McArthur, for her dedicated support and guidance.

I wish to show my gratitude to Prof. Robert Biddle and Dr. Audrey Girouard for all their generosity, and also other faculty members of human-computer interaction at Carleton University, I sincerely thank you all from the bottom of my heart. 


\section{Contents}

Abstract

Acknowledgements iv

List of Tables vii

List of Illustrations viii

1 Introduction $\quad 1$

1.1 Motivation . . . . . . . . . . . . . . . 3

1.2 Research Questions . . . . . . . . . . . . . . . 4

1.3 Methodology .................... 5

1.4 Thesis Outline . . . . . . . . . . . . . . . . 6

1.5 Associated Publications . . . . . . . . . . . . 7

2 Literature review $\quad 9$

2.1 Music Theory . . . . . . . . . . . . . . . . . . . . . 9

2.1.1 Musical Instrument Digital Interface . . . . . . . . 11

2.2 Automatic Page-turner . . . . . . . . . . . . . . . . . 13

2.2.1 Mechanical Page-turners . . . . . . . . . . . . . . 13

2.2.2 Digital Page-turners . . . . . . . . . . . . . . . 14

2.2.3 Page-turners for Musicians . . . . . . . . . . . . . 17

2.3 Score Following . . . . . . . . . . . . . . . . . . . . . . . . . 19

2.3.1 Audio-based . . . . . . . . . . . . . . . 19

2.3.2 Video-based . . . . . . . . . . . . . . 21

2.3.3 Miscellaneous ................... . . . . . 24

2.4 Conclusion . . . . . . . . . . . . . . . . 25

3 Design $\quad 26$

3.1 Video processing limitations . . . . . . . . . . . . . . 27

3.2 Wearable Technology Approach . . . . . . . . . . . . 29

3.2.1 Equipment. . . . . . . . . . . . . 30 
3.2.2 Acceleration . . . . . . . . . . . . . . 34

4 Methodology $\quad 39$

4.1 Research Questions . . . . . . . . . . . . . . . . . . . . 40

4.2 Data Collection . . . . . . . . . . . . . . . . . . 41

4.3 Data Analysis . . . . . . . . . . . . . . . . . . . 47

4.3.1 Time Series Alignment . . . . . . . . . . . . . . . . . 47

4.3 .2 Data Clustering . . . . . . . . . . . . . . . . . . 51

4.3.3 Signal Peaks Finding . . . . . . . . . . . . . 54

4.4 Evaluation . . . . . . . . . . . . . . . . . 57

4.4 .1 Alignment Types . . . . . . . . . . . . . . 57

4.4 .2 Error Metrics . . . . . . . . . . . . . . . 58

4.4 .3 Measure Labelling . . . . . . . . . . . . . . . . 61

4.4.4 Decision Model . . . . . . . . . . . . . . 64

5 Tuning the Automatic Page-turner $\quad 66$

5.1 Time Series of Digital Notes $(\mathrm{TN}) \ldots \ldots$. . . . . . . . 68

5.2 Time Series of Pressed Keys (TP) . . . . . . . . . . . . . 68

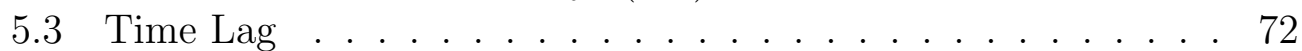

5.4 Time Shifting . . . . . . . . . . . . . . . . . 74

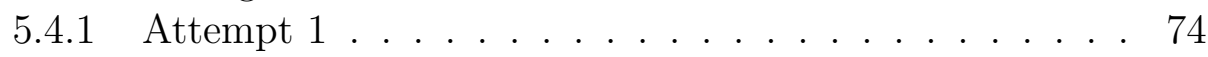

5.4 .2 Attempt $2 \ldots \ldots \ldots \ldots \ldots 75$

5.4 .3 Attempt $3 \ldots \ldots \ldots \ldots 76$

5.5 Online Mode . . . . . . . . . . . . . . . . . . 78

5.5.1 Data Stream . . . . . . . . . . . . . 79

$\begin{array}{llr}6 & \text { Results } & 82\end{array}$

6.1 WT-gesture . . . . . . . . . . . . . . . . . . . . . . . . . . . . 83

6.2 Peak Detection . . . . . . . . . . . . . . . . . . 84

6.3 Time Shifting . . . . . . . . . . . . . . . . . . . 86

6.4 Decision Model . . . . . . . . . . . . . . . . . . . . 88

6.5 Online Mode . . . . . . . . . . . . . . . . . . 91

$\begin{array}{lll}7 & \text { Discussion } & 92\end{array}$

$\begin{array}{llr}8 & \text { Conclusions } & 97\end{array}$

8.1 Limitations . . . . . . . . . . . . . . . . . . . . . . . . . . . . . . . . . . . . . . . . . . . .

8.2 Future Studies . . . . . . . . . . . . . . . . 100

$\begin{array}{ll}\text { Appendices } & 101\end{array}$

$\begin{array}{ll}\text { Bibliography } & 105\end{array}$ 


\section{List of Tables}

2.1 A representation of notes in the MIDI standard . . . . . . . 11

4.1 Details of played music pieces in experimental sessions. . . . . 42

4.2 Default Configuration of NGIMU device for this study . . . . 43

4.3 The steps of applying cross-correlation function to $f, g$ series. . 50

6.1 DTW distances (dd) and alignment errors (err) for all runs with different time-shifting approaches. . . . . . . . 86

6.2 Shows different DTW alignment types for all runs with different time-shifting approaches. $(\mapsto)$ symbol represents the one-to-one alignment, $(\leftrightarrow)$ symbol shows the fully aligned connection, and $(\rightarrow)$ symbol indicates the one-to-many connections. . . . . . . . . . . . . . 87

6.3 The necessary data for decision making for turning page at the middle of each piece (offline mode). The $\mapsto, \leftrightarrow$ symbols represent one-to-one and aligned connections respectively. . 88

6.4 The necessary data for making decision for turning page at the middle of the each piece in online mode. . . . . . . . . . 91 


\section{List of Illustrations}

2.1 Notation of similar notes in the Treble, Alto, and Bass clefs. . 10

2.2 Showing the boundaries of four measures of piano sheet music. 10

2.3 PageFlip-Lite in different phases of page turning. . . . . . . . 14

2.4 AirTurn DUO 200 digital page-turner. . . . . . . . . . . . 16

2.5 The result of applying video movement detection on two frames. 23

3.1 Schematic diagram of my proposed system. The components and the connections between them. . . . . . . . . . . . 29

3.2 The possible movements on an object in 6 degrees of freedom. 31

3.3 NGIMU module with the plastic container and body strap. . . 32

3.4 The oscillation of the moving accelerometer in the $x$ axis, from positive to negative direction and vice versa. . . . . . . . 35

3.5 Acceleration noises in idle mode $(50 \mathrm{~Hz}, 20$ seconds $) . \quad$. . . . . 36

3.6 Simple moving average (a) and Exponential moving average (b) filters applied to the acceleration data. . . . . . . . 38

4.1 The three-dimensional axes reference in this study. . . . . . . 44

4.2 The wrist-twisting gesture detector detected the boundaries of valid data. . . . . . . . . . . . . . . . . . 46

4.3 Cost matrix schema for solving DTW problems. . . . . . . . 48

4.4 The results of DTW function on two series. The digits inside the matrix show the local distances and the blue line indicates the optimum path. . . . . . . . . . . . . . . 48

4.5 Cross-correlation function applied to series A,B with the range of lags $[-1,+2] . \ldots \ldots \ldots \ldots \ldots$

4.6 Five iterations of K-means clustering function for a dataset when $k=3$. Each symbol represents the cluster centroids and the lighter symbols represent the members of each cluster. . . 53

4.7 Shows signal peak clustering with K-means algorithm (Left) and Geometric angle (Right with angle $\left.<5^{\circ}\right) . \ldots . . .256$

4.8 DTW alignment types. . . . . . . . . . . . . . 58 
4.9 Straight line between two points can be considered as a hypotenuse of a right triangle, and its length can be obtained by Pythagorean theorem. . . . . . . . . . . . . 59

4.10 Shows the Manhattan distance sample in which the distance of two points is equal to the length of horizontal and vertical distances. . . . . . . . . . . . . . 60 60

4.11 Shows points in two-dimensional space. The cosine of $\theta$ determines the relative distance between points. . . . . . . . . . . 61

4.12 Show the first type of chunk alignment, the pianist played all notes. . . . . . . . . . . . . . 62

4.13 The second type of chunk alignment, pianist did not play most or all notes of the chunk. . . . . . . . . . . . 63

4.14 The third type of chunk alignment, pianist played extra notes. 63

4.15 Decision model flowchart. . . . . . . . . . . . . . . 64

5.1 Workflow of this study in experimental sessions. . . . . . . . . 67

5.2 Time series of digital notes for Twenty-one Pilots piece with the MIDI values. . . . . . . . . . . . . . . . . . . . . . . 69

5.3 Data preparation for peak detection process. . . . . . . . . 70

5.4 A Heuristic filter is applied to the raw accelerometer signal to keep the peaks and reduce the unnecessary data points. . . . . 71

5.5 Shows the gyroscope (left) and the accelerometer (right) data of the same record. The WT-gesture detection guideline used for accelerometer to find the first pressed key. . . . . . . . . 73

5.6 The top plot shows the result of applying DTW to TN and TP series. The bottom plot is the zoomed of the top one. . . . 75

5.7 The top plot shows the DTW function applied to the equal chunks of TN and TP series. The bottom plot is the zoomed of the top one. . . . . . . . . . . . . . 76

5.8 The top plot shows the result of applying the DTW function to the dynamic chunks of TN and TP collection. The bottom plot is the zoomed of the top one. . . . . . . . . . 77

5.9 Workflow of automatic page-turning in online mode. . . . . . . 78

5.10 Original signal (top) and the same signal in the buffered mode (down). . . . . . . . . . . . . . . 80

5.11 Four steps of filtering the accelerometer signal to detect signal peaks in online mode. . . . . . . . . . . . . . . 81

6.1 Shows the normalized number of acceleration data after applying WT-gesture function for all played pieces. . . . . . . . . 83 
6.2 Shows the percentage of detected peaks to actual music notes with K-means method. . . . . . . . . . . . . . . . . . . 84

6.6 The final alignment of Adagio for all runs. Red shapes indicates the turning-page points. . . . . . . . . . . . . . 89

6.7 The final alignment of Golden Dreams for all runs. Red shapes indicates the turning-page points. . . . . . . . . . . . 89

6.8 The final alignment of Rondo Alla Turca for all runs. Red shapes indicates the turning-page points. . . . . . . . . . 89

6.9 The final alignment of Sonatina Op. 20 for all runs. Red shapes indicates the turning-page points. . . . . . . . . . 90

6.10 The final alignment of Twenty-one Pilots for all runs. Red shapes indicates the turning-page points. . . . . . . . . 90 


\section{Chapter 1}

\section{Introduction}

Book is the most common type of paper-based medium that stores information in both written and visual forms, which consists of several pages and a cover that fastens all pages together. The book structure (or other forms of documents such as newspapers, magazines, brochures, etc.) is not designed to stay open on its own vertically, so readers usually use one or two hands to keep it open. However, some readers prefer to use book-holders or putting them on a flat surface while reading.

Page-Turning is a necessary act in reading documents and requires at least one hand to fulfill. This simple task that we usually take for granted could be very challenging in several situations. For instance, people with physical or neurological disorders or any other types of disabilities that involve motor functions such as upper limb dysfunctions[1] can have severe difficulties in turning book pages. Page-turning can also be more challenging when both hands are occupied in another task. For example, musicians need both hands to play musical instruments. Most of the musicians and students can sight- 
$\operatorname{read}^{1}$, so they must use one hand to turn the music sheets, and this action causes a short delay or distraction.

Although professional pianists may play the entire piece by heart, for complex parts they prefer to sight-read notes. At concerts, another musician usually stays close to the pianist, following the musical notes and turning music sheets at the right intervals. This solution may work in a recitals ${ }^{2}$ once a year, but pianists can not have a person in their practicing time or whenever they need. This question also applies to piano learners.

This study suggests a novel idea for a new automatic, and hands-free page-turner solution for pianists to help them during their live performances as well as their practice time.

\footnotetext{
${ }^{1}$ Sight-reading is the practice of reading and performing a piece of music that the musician has not seen or learned before.

${ }^{2} \mathrm{~A}$ recital is a type of concert that has only one musician or one musician and an accompanist.
} 


\subsection{Motivation}

The idea of creating a hands-free page-turner for musicians is not a new topic, and the first patent dates back to 1970s[2]. The early models of page-turners worked with mechanical components, and there was no automation in their processes. The high cost of mechanical parts and low performance were addressed as significant drawbacks of this generation of page-turners[1]. However, with the advancement of technology and computers in recent decades, researchers have proposed digital solutions such as audio and video processing techniques $[3,4,5]$ to solve the page-turning problems.

Last decade witnessed a significant trend of studies on wearable devices as a new way of interacting between humans and computers in academia[6]. The combination of wearable gadgets mainstream, artificial intelligence, and improvements in wireless communications made wearable devices as an excellent alternative to existing solutions in various domains [7, 8].

To develop a new solution for solving page-turning problems, I came up with using wearable devices rather than video and audio processing methods. In this approach, pianists should wear a pair of wearable gadgets on their wrists, which capture the sequences of the wrists' up and down movement — when the pianist presses keys - and sync them to digital notes. This synchronization provides some information regarding which part of the piece is played and whether it can turn the digital sheet music at predefined points. 


\subsection{Research Questions}

Piano uses the key-action mechanism, which transfers the finger pressure on the keys to the hammers, and hammers create sound by hitting the strings. The force direction for pressing a key is top to bottom, so the wrist moves slightly in the same direction. The proposed method utilizes a wearable device equipped with inertial measurement units that sense motions in threedimensional space.

This study has two primary questions as follows:

- How can we track the pianist's wrists oscillatory motions (up and down) and track the sequences of these motions?

- How shall we synchronize the series of oscillatory motions to the sequences of digital notes to validate the pianist's performance and decide to turn the digital music sheet at the right time? 


\subsection{Methodology}

As stated earlier, an inertial measurement unit senses hand movement with its accelerometer sensors. I found that pressing the piano keys makes spikes with high-value peaks in the acceleration signal. By assuming this phenomenon as a reference, the sequences of acceleration spikes are equal to the sequences of pressed keys. I called these arrays as time series of pressed keys, and it is the first data type of this study.

Designing an automatic page-turner also needs another series (as an expected reference) to compare to pressed keys sequences. This comparison indicates the orders of played notes by musicians and evaluates the musician's performance. The MIDI format is selected for this stage, which contains all the necessary data - start time and duration of each note - for creating a time series of digital notes.

The synchronization process of both mentioned time series has been done with dynamic time warping and a heuristic method for chunking time series into smaller groups. Also, the cross-correlation function resolved time latency between aligned pairs. Three different metrics were proposed for evaluating aligned pairs that specify each chunk's status, alignment types, and the alignment error rates. Finally, a custom decision model evaluates the nominated chunks' data for page-turning and decides to turn the digital sheet music.

The proposed system tries to remove the previous studies' complexity and focused more on using wearable technologies and their potential features to solve the page-turning problems. 


\subsection{Thesis Outline}

The next chapter discusses music theory, music notation systems, and MIDI format. It then reviews the mechanical and digital page-turners and finally explains the score-following system's background and methods.

Chapter 3 describes the limitations of previous studies in the page-turning and score-following domains. The wearable technologies as an alternative solution will be discussed, and the primary hardware of this research will be presented.

Chapter 4 explains the research methods and declares the research questions, data collection processes and data analysis methods. Finally, a heuristic evaluation methods will be explained.

Chapter 5 investigates the characteristics of two primary time-series of this study, and then, the problems of aligning them will be discussed. In the last section of this chapter, the proposed design for the automatic pageturning will be explained.

Chapter 6 presents the results of the experimental sessions.

Chapter 7 discusses results, limitations and significant problems. Ultimately, in Chapter 8, we will have a conclusion. 


\subsection{Associated Publications}

Seyed Ali Mirazimzadeh and Victoria McArthur. Automatic page-turner for pianists with wearabmotion detector. In International Conference on Human-ComputInteraction, pages 209-218. Springer, 2020 


\section{List of Abbreviations}

CC: Cross-correlation

DTW: Dynamic time warping

EMA : Exponential moving average

FFT : Fast Fourier transform

FT : Fourier transform

IMU : Inertial measurement units

MIDI : Musical instrument digital interface

RMSE : Root mean square error

SMA : Simple moving average

TP:Time-series of pressed-keys

TN:Time-series of digital notes

WT: Wrist-twisting

WTG: Wrist-twisting gesture 


\section{Chapter 2}

\section{Literature review}

\subsection{Music Theory}

The method and style of writing melodies and rhythms are as old as history. Music, like other forms of art, has been matured over centuries. The Catholic Church founded the modern notation system in medieval Europe to ease the music integration among churches. This system developed later in the Renaissance and Baroque era. The current music notation standard uses various symbols for coding music attributes. These codes show pitches (high and low of the notes), tempo (speed), articulations of notes, and even different playing forms for specific instruments. Although we have a unified standard for music notation, many folklore music styles use unique symbols to express their cultural beliefs and feelings. [10, 11]

In the notation system, vertical lines (bar-line) divide staves into equal chunks (measure). Each measure satisfies the specified time signature. For example, in the $4 / 4$ time signature, each measure should contain the four 


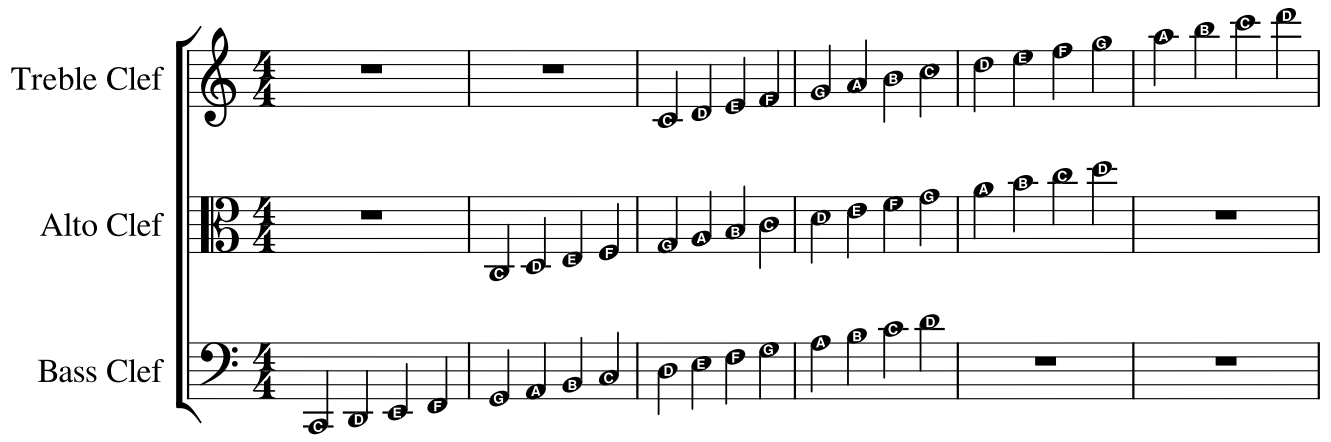

Figure 2.1: Notation of similar notes in the Treble, Alto, and Bass clefs.

quarter-note beats (or the equivalent rest ${ }^{1}$ ). Since the length of all measures is equal, we can use them as the right unit for calculating time sequences (see fig. 2.2).

${ }^{1} \mathrm{~A}$ rest is a musical notation sign that demonstrates the absence of a sound.

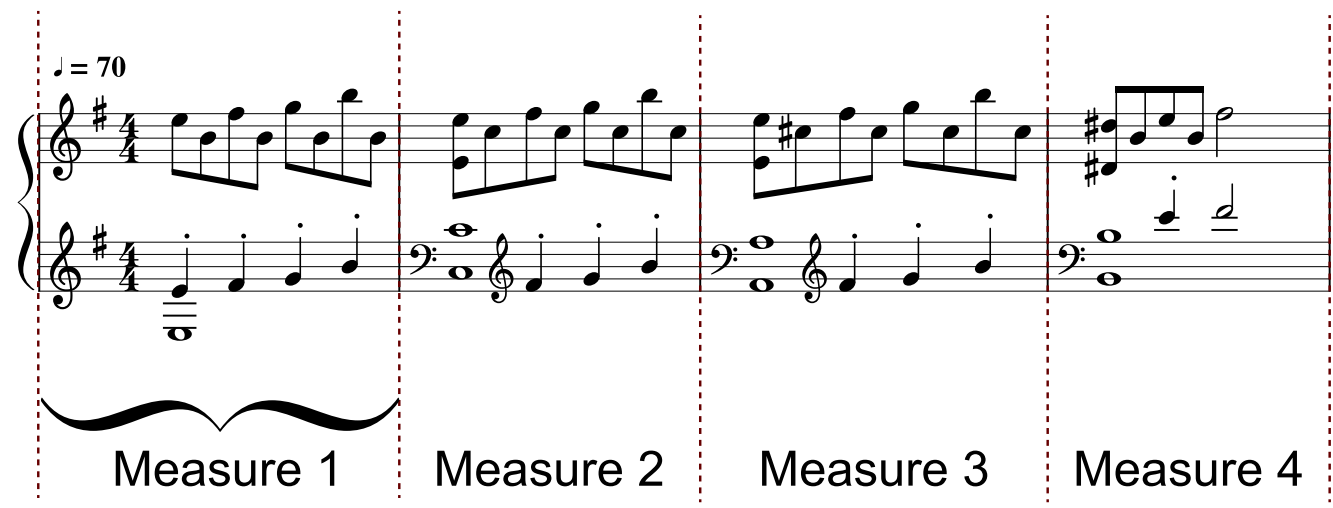

Figure 2.2: Showing the boundaries of four measures of piano sheet music. 


\subsubsection{Musical Instrument Digital Interface}

Musical instrument digital interface (MIDI) is a standard communication protocol for storing and transmitting digital music information[12]. MIDI serves as a unified language for digital music equipment like synthesizers, controllers, and computers to facilitate communication. Unlike the analogue system that transfers sound signals, MIDI transfers the event data (messages), which contains the notes playing time, duration, pitch, velocity, after-touch, knob movement. Digital MIDI supported synthesizers can convert messages to sound signals. Using this feature, we can simulate the sounds of various instruments such as piano and cello with a piece of MIDI.

MIDI protocol covers more than 10 octaves and 127 notes. Starts from 0 (note $\mathrm{C}, 8.175 \mathrm{~Hz}$ ) to 127 (note $\mathrm{G}, 12.543 \mathrm{kHz}$ ). Fig 2.1 shows details of all supported music notes in the MIDI standard.

\begin{tabular}{|c|c|c|c|c|c|c|c|c|c|c|c|c|}
\hline \multirow{3}{*}{ Octave } & \multicolumn{12}{|c|}{ Note Number } \\
\hline & Do & Do & $\operatorname{Re}$ & $\operatorname{Re} \#$ & Mi & $\mathbf{F a}$ & Fa\# & Sol & Sol\# & La & La\# & $\mathrm{Si}$ \\
\hline & $\mathrm{C}$ & C\# & D & $\mathrm{D} \#$ & $\mathbf{E}$ & $\mathbf{F}$ & $\mathbf{F} \#$ & G & G\# & A & $\mathbf{A \#}$ & B \\
\hline 0 & 0 & 1 & 2 & 3 & 4 & 5 & 6 & 7 & 8 & 9 & 10 & 11 \\
\hline 1 & 12 & 13 & 14 & 15 & 16 & 17 & 18 & 19 & 20 & 21 & 22 & 23 \\
\hline 2 & 24 & 25 & 26 & 27 & 28 & 29 & 30 & 31 & 32 & 33 & 34 & 35 \\
\hline 3 & 36 & 37 & 38 & 39 & 40 & 41 & 42 & 43 & 44 & 45 & 46 & 47 \\
\hline 4 & 48 & 49 & 50 & 51 & 52 & 53 & 54 & 55 & 56 & 57 & 58 & 59 \\
\hline 5 & 60 & 61 & 62 & 63 & 64 & 65 & 66 & 67 & 68 & 69 & 70 & 71 \\
\hline 6 & 72 & 73 & 74 & 75 & 76 & 77 & 78 & 79 & 80 & 81 & 82 & 83 \\
\hline 7 & 84 & 85 & 86 & 87 & 88 & 89 & 90 & 91 & 92 & 93 & 94 & 95 \\
\hline 8 & 96 & 97 & 98 & 99 & 100 & 101 & 102 & 103 & 104 & 105 & 106 & 107 \\
\hline 9 & 108 & 109 & 110 & 111 & 112 & 113 & 114 & 115 & 116 & 117 & 118 & 119 \\
\hline 10 & 120 & 121 & 122 & 123 & 124 & 125 & 126 & 127 & & & & \\
\hline
\end{tabular}

Table 2.1: A representation of notes in the MIDI standard

Standard MIDI File (SMF) is a computer file format that carries MIDI events in binary forms, and many operating systems can parse that. MIDI 
files only contain text-based messages, so their size is much less than regular music formats like MP3s, WAVs, and M4As that have the compressed audio content. Almost all smart devices have a virtual MIDI interface that can interpret MIDI messages and transform them into sound.

For this research, I picked MIDI as a digital music format. MIDI is powerful, flexible, and extensible enough to represent all the things I want to follow. Also, parsing them with most of the programming languages is straightforward. Additionally, several free or open-source score editors like MuseScore[13] support this file format. 


\subsection{Automatic Page-turner}

For reading books, turning pages is a necessary and effortless act that readers must do, and it requires at least one hand to fulfill. Numerous limitations can disrupt this process, including neurological disorders, spinal cord injuries, amyotrophic lateral sclerosis (ALS), or other disabilities that involve the motor functions or upper limb dysfunction[1]. In other cases, the mentioned process can be more challenging if both hands have already been occupied with performing another task. For instance, pianists need both hands to play and turning the page can cause a short delay. Various academic disciplines have addressed these issues and suggested the automatic hands-free pageturning mechanism as an assistive technology[14, 15].

The automatic page-turner is a mechanical or digital mechanism that automates the process of turning pages forward or backward[1].

\subsubsection{Mechanical Page-turners}

Almost all mechanical page-turners need three essential steps as engagement, transport, and restraint[1] to turn a page. In the engagement step, the mechanical parts grab a page and lift it away from the other pages with various mechanisms such as suction tubes, adhesive, magnetic tabs, clips, or friction wheels. In the next step, the grabbed page moves to the other side of the book with different methods like rotating arms, rotating disks, spring mechanism, or linear movable arms[1]. Eventually, like in the real world, after moving, the moved page should be fixed in place by the restrainer component (see fig. 2.3). 

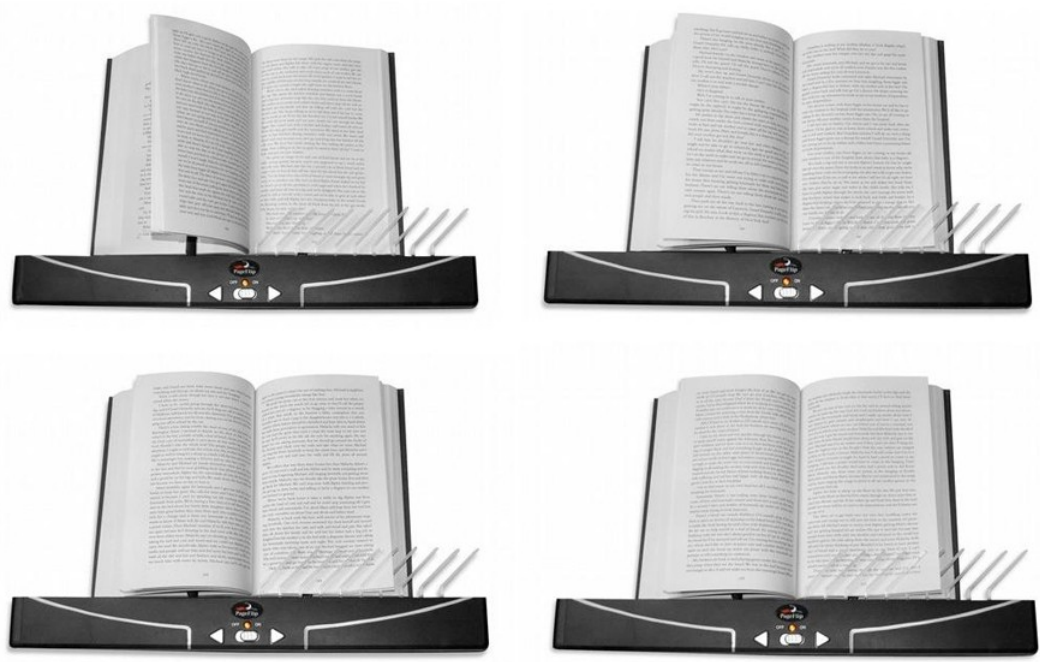

Figure 2.3: PageFlip-Lite in different phases of page turning.

Mechanical page-turners use push-buttons to move pages back and forth, and manufacturers usually mount these buttons on the bottom side of the device (fig. 2.3). Pushing these buttons creates similar restrictions as the turning page does with one hand. Also, paper-based documents are available in different sizes, and adjusting mechanical parts for each of them is a timeconsuming process. The high cost of mechanical pieces, low performance, and noise of components are the major drawbacks of this type.

\subsubsection{Digital Page-turners}

Before discussing digital page-turners, it is necessary to clarify the meaning of digital content. With the improvements in electronic science, the data of many mediums have been converted into digital format. E-books ${ }^{2}$ may be one of the most notable evidence of this evolution. Today, publishers cre-

\footnotetext{
${ }^{2}$ Electronic books
} 
ate electronic books with software, so e-books are initially created digitally. For older books, this process is divided in two steps. First, the high-speed scanners scan each page of books separately and save them as digital images on computers. In the next step, the $\mathrm{OCR}^{3}$ or $\mathrm{OMR}^{4}$ software extract the contents of each page and convert them to machine-encoded text or digital music data.

The mentioned applications save digital content into specific blocks in computer files. When users open files with the file viewer, the application loads each block separately and displays that on screen. Users can easily change the order of contents with simple input devices like keyboards. File viewers usually get the page switching command with the keyboard arrow keys, page-up/page-down, or space/enter keys. Some viewers also use visual effects (e.g. with the page flipping effect) during the changing pages to create similar feelings of page-turning in the real world, but apps only load the next block contents behind the scene.

Unlike mechanical page-turner devices, which can work independently, digital page-turners need computers or similar digital devices to display contents. In other words, instead of using paper, lighter and portable gadgets like tablets can be used. In the music domain, tablets with a large screen are the fittest option to paper-based music sheets[16].

Digital page-turner devices also have mechanical push buttons, usually as foot pedals. In other words, digital page-turner devices are not much more than a keyboard with two keys. So, each pedal sends a specific signal

\footnotetext{
${ }^{3}$ Optical character recognition

${ }^{4}$ Optical music recognition
} 
with different methods to the computer for switching content. For example, most commercials digital page-turners like Airturn ${ }^{5}$ (see fig. 2.4), PageFlip ${ }^{6}$, $\mathrm{STOMP}^{7}$, or Donner ${ }^{8}$ have the Bluetooth or $\mathrm{USB}^{9}$ wired interfaces for connecting to other devices.

With the above explanations, we can accept that digital page-turners solved the significant problems of mechanical ones. However, we must keep in mind that digital page-turners are not a single device and require additional computers or tablets to perform.

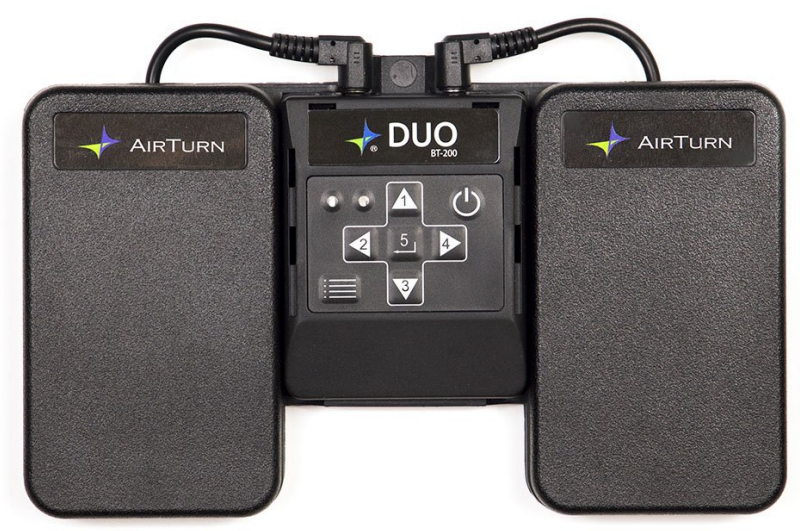

Figure 2.4: AirTurn DUO 200 digital page-turner.

\footnotetext{
${ }^{5}$ www.airturn.com

${ }^{6}$ www.pageflip.com

${ }^{7}$ www.codamusictech.com/products/bluetooth-page-turner-music-pedal-for-tablets

${ }^{8}$ www.donnerdeal.com/products/donner-bluetooth-page-turner-pedal-for-tablets-ipadrechargeable

${ }^{9}$ Universal Serial Bus
} 


\subsubsection{Page-turners for Musicians}

For almost all instruments, musicians need both hands to play. In some instruments such as violin or guitar, the left hand fingers ${ }^{10}$ depressing strings at specific points ${ }^{11}$ and the right hand vibrates the string to generate sound. In other instruments like piano, each hand acts separately and plays different notes with a different rhythm and speed. For complex instruments like drums or organs, not only hands are required to play, but also feet operate different pedals. With this introduction, we can understand that using both hands for playing is mandatory throughout the play, and other tasks like page-turning can cause distraction.

Before the invention of automatic page-turners, musicians utilized different solutions to overcome the page-turning problem. For example, professional music editors would set long notes or pauses (rests) at the end of each page[5], so the musician had a short time to turn pages. Another approach was hiring a skillful person (called a page-turner) to sit close to the musician to follow notes during the performance and turn the page at the right time. Finally, the permanent solution was memorizing all notes by the musician, so there was no need of using music notes.

Machnacz[2] invented the first mechanical page-turner for musicians in 1972; however, the basis of today portable page-turners is designed by Carr and Lucero in 1992[17]. Lucero's device had foot pedals for switching pages and could be mounted in music stands. Although the proposed design used

\footnotetext{
${ }^{10}$ For the left-handed people the order is opposite

${ }^{11}$ In some instruments like guitar, this place is called "fret" and in fret-less instruments like violin and cello is called "stop"
} 
foot pedals to solved the pressing buttons by hand, it brought a new problem. Piano instruments have three built-in pedals for adjusting tones, and adding two more pedals for turning pages can make confusion. On the other hand, for musicians, whether they are standing or sitting, maintaining the balance is essential, and any sudden changes in foot position for pressing pedals might affect their balance[16].

Researchers conducted several studies to eliminate the direct user interaction (e.g. foot pedals) with page-turners, so they suggested some alternative methods, which I will explain in the next section. 


\subsection{Score Following}

Score-following refers to the process of tracking the musician play (mostly in audio signals) in order to align it with actual music notes[18]. Some literature also addressed score-following as the automatic accompaniment systems and focused on the role of the computers in analyzing data. The first idea was suggested in 1984 with three steps for detecting and processing the performer inputs, aligning with expected patterns, and handling the timing issues[19, 20].

In this section, I will review the two primary approaches of score-following.

\subsubsection{Audio-based}

The first approach uses the audio processing techniques to detect music contents in two phases as audio feature extraction and alignment[21]. These methods try to extract musical attributes such as pitches, tempos, vocal speech and vowels, or even instrument types in sound signals[22, 23]. We should notice that analyzing the recorded audio signals is a complex task and several details like bias, noises, and low-quality hardware can modify the signals and affect result.

In the audio feature extraction phase, applying the mathematical equations directly to the raw audio signals is not easy, so it must be transformed into a mathematical object. For several years, researchers utilized Fourier transform (FT) functions as the primary method for analyzing the monophonic and polyphonic ${ }^{12}$ sound signals[24, 25, 26, 27].

\footnotetext{
${ }^{12} \mathrm{~A}$ type of audio signals that contain two or more independent melody
} 
The FT function is a potent mathematical tool that provides an alternative view of the signal, which challenging problems in signal scope become very simple to analyze. FT transforms the signal time-domain to the frequency-domain. In other words, the time-domain signal shows the changes in data over time, but the frequency-domain represents the part of the signal that exists in a particular frequency band within a range of frequencies (spectrum).

This function prepares signals for other operations like filtering. For example, the frequency spectrum shows the noise frequencies, so after detecting and removing specific frequencies, the filtered data can be transformed again into the time-domain (signal) with the inverse Fourier transform function.

As mentioned earlier, extracting music contents from raw sound signals (by FT or similar methods) has some limitations. Therefore, researchers have become more motivated to focus on the second phase (alignment) in the score-following problems. In this approach, researchers compare two recorded audio signals. The first record is a reference and the second one is a piece played by a musician (study participants). The comparison algorithms work in both online (real-time) and offline modes. The reference could be a digital recording that is being played by musicians[25], or an audio file which is generated by computer (via MIDI synthesizer)[24], or both[28]. Most of the alignment methods in this domain compute distances between actual and recorded values to find the optimum path among pairs (see section 4.3 .1 for more details). 


\subsubsection{Video-based}

Technological improvements have made electronic devices more accurate and faster, and also cheaper. Almost all modern gadgets like smartphones, tablets, smartwatches have high-speed processors, wireless connection interfaces, highresolution cameras, and advanced operating systems. The high-tech package of hardware and software, besides their accessibility, have led researchers to utilize more electronic devices in their studies.

Computer vision (video processing) is an interdisciplinary scientific field that attempts to obtain computer-based understanding by acquiring, processing, and analyzing digital images. Video processing takes more time to process due to the larger volume of data rather than audio processing. However, high-performance hardware and open-source projects[29, 30, 31] have facilitated the research path in this field.

On an abstract level, the raw video files (not compressed) are nothing more than the aggregation of several digital images in a row. Storing all frames requires a large amount of space, so several methods (Codec) help encoding/compressing video frames. Every time, video players decode/decompress the contents to the original image and show them on screen. The general approach for video compression algorithms is almost the same. They try to find similarities ${ }^{13}$ from one frame to another and mark them as a repeater. With this definition, we can keep the first reference, and eliminate the rest repeated labels in the next frames.

The basic strategy in video processing is continuously analyzing frames

\footnotetext{
${ }^{13} \mathrm{~A}$ Pixel is the smallest addressable part of the digital image.
} 
over time. For instance, the basic movement detection algorithms measure two pixel distances by calculating the square root of $\mathrm{RGB}^{14}$ codes for each pixel. The zero distance values represent the equality of pixels in two frames; therefore, no movement is captured for those pixels. A simple comparison of two video frames is shown in fig. 2.5 , which dark pixels in fig. $2.5 \mathrm{c}$ describes no movements (zero distance) in contrast to white areas that had positive distances. The mathematical notation for measuring distance is denoted in 2.1, that $R, G$, and $B$ are the range of red, green, and blue code, and $N$ is the frame number.

$$
\text { distance }=\left(R_{(n)}-R_{(n-1)}\right)^{2}+\left(G_{(n)}-G_{(n-1)}\right)^{2}+\left(B_{(n)}-B_{(n-1)}\right)^{2}
$$

I previously stated that compression algorithms reduce video file sizes. In many cases, the compression stage happens in real-time (while recording by hardware), and the final frames quality are not as sharp as fig. 2.5. Thus, there is a need for utilizing digital filters to change the frame colour balance, hue, saturation, brightness, exposure etc. for normalizing and making frames comparable.

Computer vision approaches have been widely used in several studies for tracking the movements of the musician's hands, feet or even eyes. The educational domain was addressed as the objective of some studies[32, 33] specifically when the tutor and student are not in the same physical places, and tutors use distance learning methods. Learning playing piano is difficult

\footnotetext{
${ }^{14} \mathrm{RGB}$ is a famous additive colour model in electronic devices, that uses a different range of Red, Green, and Blue colours for reproducing a broad range of colours. Each colour has an integer value in a range of $0 \rightarrow 255$, so the all possible combination is around $256 \cdot 256 \cdot 256 \simeq 16.7$ million colours
} 
and requires several years of practice and requires constant supervision[34], using computer vision facilitates this process for detecting errors, specifically in hand positioning[33]. Other works reveal that researchers used different methods for tracking hands or fingers in video content by polishing fingernails with different colours to get real-time and quick detection[35]. Oka et al.[36] investigated on using depth cameras for detecting fingers. Tang et al.[37] tracked the users head gestures with a computer vision. Their system initially captured the participants faces, and the facial landmark tracking method detected changes. Kora[16] used Google Glass as a wearable device to identify the head motions and winks as a gesture for triggering the page-turning action. Tabone[5] worked on an automatic page-turning system based on the gaze tracking design. The proposed system used a dark pupil eye-tracking module with an infrared illumination tool (for emitting light in the infrared spectrum).

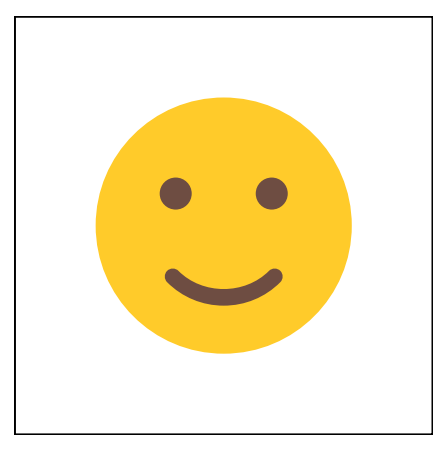

(a) Image of frame $n-1$

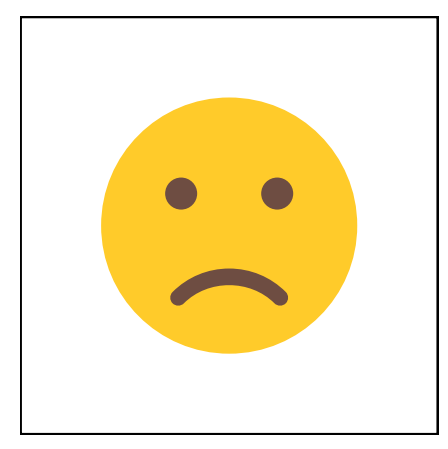

(b) Image of frame $n$

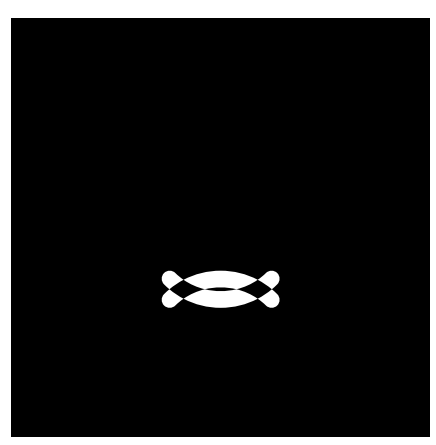

(c) Difference distance

Figure 2.5: The result of applying video movement detection on two frames. 


\subsubsection{Miscellaneous}

The scope of science is limitless, and multiple solutions can solve one problem. In the score-following area, audio and video-based approaches are prevalent; however, researchers have developed innovative methods. Bevilacqua et al. [38] proposed a wearable gadget for monitoring the violinist hands inertial movements and a sensor for detecting the bow pressure. Mitchell et al.[39] used a combination of gloves and IMU $^{15}$ devices for detecting the musician hand gestures.

\footnotetext{
${ }^{15}$ Inertial measurement unit
} 


\subsection{Conclusion}

The idea of using manual or digital page-turners for musicians is not new, and many scientific studies have been conducted to solve the page-turning problem for more than 50 years. In the beginning, the page-turner systems operated mechanically, and with the advent of computers, more electronic equipment has been utilized.

The overall results of studies in score-following and related areas revealed that musicians might have different perceptions of music, which affects the performance in terms of articulation and time management. In other words, each musician has a unique style of playing, and very slight variations in tempo or rhythm will likely be observed each time[28, 40].

By reviewing the literature, we can conclude that score-following systems are still prone to error and need more investigations. 


\section{Chapter 3}

\section{Design}

In the previous chapter, I explained the critical role of audio and video processing techniques in score-following and page-turning domains. Since recent studies mostly focused on video-based methods, first, I will describe the reported limitations and concerns of using the video processing method. Then I will explain my proposed design, which uses wearable technologies to cope with page-turning problems. Finally, I will explain the primary component of my suggested design and its characteristics in detail. 


\subsection{Video processing limitations}

Some issues such as the expensive hardware, and the risk of using electronic devices with batteries in live performances were addressed in literature as the general drawbacks of digital page-turners[1].

One of the notable reported issues of utilizing video processing approach in academic studies is the considerable volume of data to process[41], which needs high-performance hardware and complex software packages. Although cloud computing services may resolve the mentioned barriers by offering ondemand IT resources, the privacy concerns of uploading video files to online servers still exist[42] and should not be ignored.

Object tracking is the conventional computer vision method, which consists of two central functions as object detection and movement modelling. Researchers used this method vastly in page-turning and score-following studies[32]. The accuracy of object detection function is highly dependent on video recording equipment, and any vibration of cameras or changing the distance to moving objects can affect results[43].

Light and shadows are other essential factors that can impact the process of distinguishing objects in video frames[44]. Usually, in live concerts, specific lighting equipment is used, which is not available in academic experimental studios. So, the role of light and its impact on video processing should be considered.

The second stage of object tracking methods is movement modelling, which attempts to understand and label the motions or gestures in video contents. This process can be more complicated when the system accuracy 
relies on constant observations or earlier stage results. In this case, any unpredictable user action may cause failure. For example, when an eye-gaze tracking system follows the musician's eyes interaction with music score, if the musician plays some parts by heart with closed eyes, the modelling system does not return the accurate results[45].

The barriers mentioned above do not deny the importance and effort of previous studies. This study aims to remove the complexity of previous experiments by utilizing wearable, portable and newer technologies. 


\subsection{Wearable Technology Approach}

As explained in the previous section, the primary objective of this study is to reduce/eliminate prior research complexity in the field of score following. The proposed design works with a pair of lightweight wearable devices like smartwatches, which users (pianists) should wear on their writs during the performance.

These gadgets are equipped with inertial measurement units (IMU) board, which each has an accelerometer sensor. By pressing piano keys, wrists move up and down slightly, and the accelerometer scene this movement and reflects that as spikes in acceleration signal. The trend of acceleration spikes describes the sequences of pressed keys, which adding time factor to it can be interpreted as a time series of digital notes. The section describes the characteristic IMU component in detail.

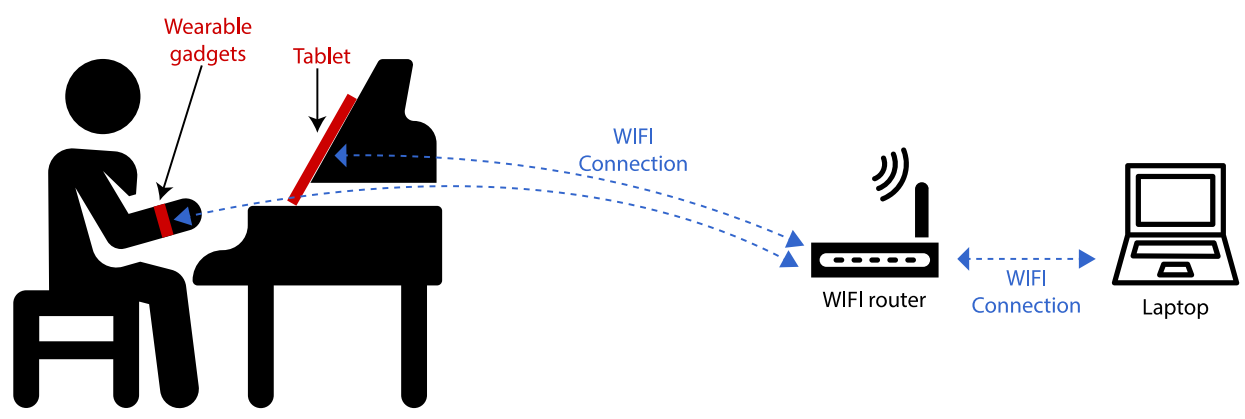

Figure 3.1: Schematic diagram of my proposed system. The components and the connections between them. 


\subsubsection{Equipment}

\section{Inertial Measurement Units}

The original model of the inertial measurement unit (IMU) appeared in the 1930s and then turned into an essential part of navigation systems later in the 1950s[46]. Due to the several limitations in size and production costs, using them in small devices was not feasible for a long time. However, advances in micro-machined electromechanical systems (MEMS) have reduced the size and increased the efficiency and accuracy of IMUs in the last decades. MEMS technologies have become more affordable than similar counterparts, and almost all modern smart electronic gadgets have with IMU sensors these days[47].

The earliest IMU models had a free-floating gyro-stabilized platform which was connected to the moving object (vehicle) by a gimbals mechanism. Any changes in vehicle orientation change the IMU body-frame, but the inertial coordinate frame remains fixed in space. So, the angle between the inertial frame and body-frame determines the vehicle orientation angle. The accelerometers are also attached to the gyro-stabilized system to measure the acceleration of the vehicle.

Today, most of the IMU devices use strap-down configuration, which means the IMU body-frame is attached rigidly to the vehicle. The attached accelerometers measure acceleration along the body frames axes, and the gyroscopes measure the angular velocity for each body-frame coordinate axes.

IMU devices have at least two sensors. A three orthogonal rate-gyroscope for measuring angular velocity and a three orthogonal accelerometer for de- 
termining linear acceleration. For more accurate results, modern IMU devices also have a magnetometer for sensing the direction and strength of Earth magnetic fields and a barometer for measuring air pressure. IMUs can track 2 to 9 degrees of freedom (DOF), which refers to the number of ways (freedom of movements) that an object can move in three-dimensional space. For instance, an IMU device with 6 DOF can sense movements along threeaxes like forward/backward on $x$ axis, left/right on $y$ axis, and up/down on $z$ axis and also roll, pitch, and rotate/yaw on the $x, y$, and $z$ axis respectively (see fig. 3.2).

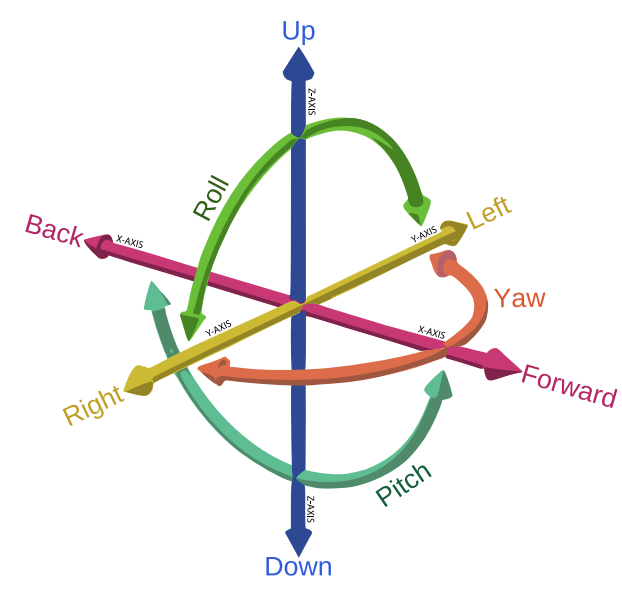

Figure 3.2: The possible movements on an object in 6 degrees of freedom.

In this research, I used a pair of NGIMU[48] electronic kits (see fig. 3.3), which have an embedded inertial measurement unit module and a real-time data logger tool and WIFI adapter. The NGIMU module is equipped with a triple-axes gyroscope, accelerometer and magnetometer sensor. All sensors send timestamps as well, which starts from zero by starting device every time. NGIMU also has several built-in sensor fusion algorithms such as attitude and heading reference system (AHRS), which can provide a drift- 
free measurement of orientation relative to the Earth. This module has a plastic housing for covering the electronic parts and a fabric body-strap that helped us to fix the plastic housing securely on the pianist wrists (for more details, check appendix 1).

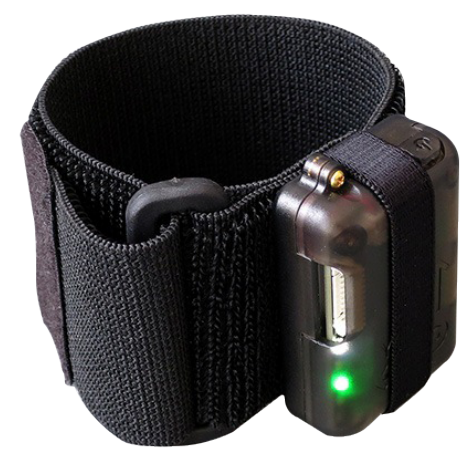

Figure 3.3: NGIMU module with the plastic container and body strap.

\section{Additional Hardware}

In experimental sessions, wearable gadgets transmitted data via a WIFI connection to central computer ${ }^{1}$. I placed a tablet ${ }^{2}$ on the piano music rack. Based on the experiment mode, the decision model generated relevant digital music sheets and transferred them to tablet to display. All experiments have been done with a 5-octave digital piano.

\section{Software \& Libraries}

I used Python[49] version 3.8.3. as the primary programming language in this study. Python is a cross-platform and high-level programming language which its simplicity and versatility plus its large open-source tools, libraries

\footnotetext{
${ }^{1}$ Lenovo laptop, CPU:Core i7,RAM:8 GB, OS:Windows 10

${ }^{2}$ Samsung Galaxy Tab A 10.
} 
and frameworks made that so popular among researchers. I used the following libraries to develop the software section of this research (for more sample check appendix 2).

- pretty_midi ${ }^{3}$ : Used for parsing MIDI files

- pandas $^{4}$ : Used for data manipulation, analysis and

- fastdtw ${ }^{5}$ : Used for providing optimal or

- $\mathrm{kmeans}^{6} \mathrm{~d}^{6}$ : Used for implementing k-means

- matplotlib ${ }^{7}$ : Used for data visualization.

- numpy ${ }^{8}$ : Used for array manipulation including mathematical,

- scipy $^{9}$ : Used for data distance calculation and signal peak

- sklearn ${ }^{10}$ : Used for data scaling in specific ranges.

I created four folders named io, core, visualization, and report to separate and categorize files in this research. I placed all functions for reading and storing data in io folder. The core folder included data preparation, synchronization, and decision functions. The files in the visualization folder generated plots and other graphic materials. The report folder held all methods for generating data for the result section. I wrote around 3500 lines of code in this study.

\footnotetext{
${ }^{3}$ http://craffel.github.io/pretty-midi

${ }^{4}$ http://pandas.pydata.org

${ }^{5}$ http://www.github.com/slaypni/fastdtw

${ }^{6}$ http://www.github.com/dstein64/kmeans1d

${ }^{7}$ http://www.matplotlib.org

${ }^{8}$ http://www.numpy.org

${ }^{9}$ http://www.scipy.org

${ }^{10} \mathrm{http}: / /$ www.scikit-learn.org
} 


\subsubsection{Acceleration}

In physics, acceleration is the rate of change in velocity over time. The acceleration equation and its unit is $a=\frac{\Delta v}{\Delta t}$ and meter per second squared $\left(m . s^{-2}\right)$. The acceleration due to gravity is a concept that is derived from Newton's Second Law of motion and the Law of Universal Gravitation, which leads to $g=\frac{G M}{R^{2}}$ equation. $g$ is the acceleration due to the gravity, $G$ is the universal gravitational constant, $M$ is mass, and $R$ is distance.

The NGIMU kit accelerometer provides raw and linear (gravity-free) acceleration data with the unit of $g$. It is necessary to note that the acceleration values are completely related to the orientation of the device in space. For example, if the IMU device is lying on a flat surface (such as a table) and its z-axis facing up, then the acceleration values of $x, y$ axes are 0 , and the value of $z$ axis will be $\approx 9.81$. By rotating the module perpendicular (parallel to the gravity) along with $x$ or $y$ axes, the value for $z$ axis would be 0 , and the accelerometer shows $\approx 9.81$ for $x$ or $y$ axes. It can be concluded that the sensors' values are not absolute, and any deviation or rotation affects data but can be adjusted mathematically.

The above fact shows that the acceleration is a vector value, which means it has a magnitude and direction. Therefore, by specifying the global coordinates and IMU's position, we can predict the direction and values of any movements. For example, any one-dimensional movements, followed by the $x$ direction $(-x \rightarrow+x)$, generate positive and then negative values (spikes) resulting from the acceleration and deacceleration phenomenon. We can expect the opposite trend with backward movements $(x \rightarrow-x)$ (see fig. 4.5). 
This phenomenon is the fundamental concept of this research, and in the next section, I will discuss several methods to identify these patterns.

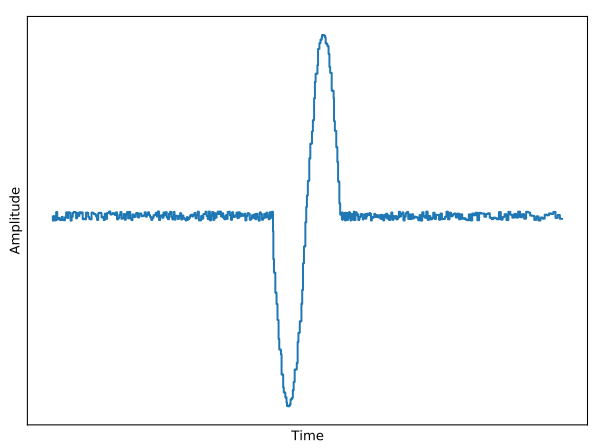

(a) Movement from $-x \rightarrow+x$

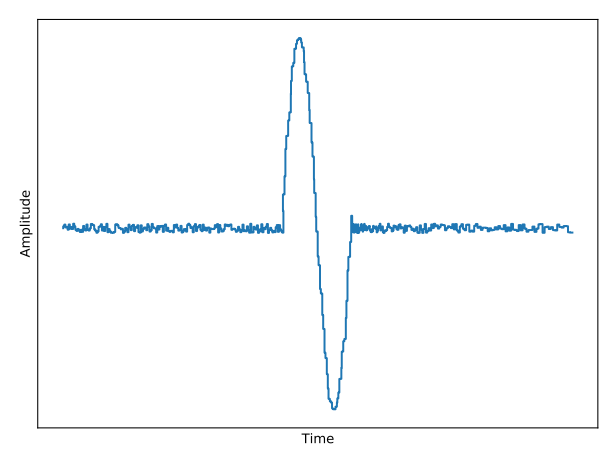

(b) Movement from $+x \rightarrow-x$

Figure 3.4: The oscillation of the moving accelerometer in the $x$ axis, from positive to negative direction and vice versa.

\section{Noise}

The noise in electronic is the result of any disturbance in voltage or current (usually low amplitude). This negative attribute is an inseparable part of electronic circuits and comes from internal or external sources. We believe that electronic modules should be able to work in a noisy environment (e.g., industrial sections), and other devices such as fluorescent, neon lamps, power lines, transmitters should not affect electronic boards. Many standards such as $\mathrm{FCC}^{11}$, require manufacturers to check and control their product electromagnetic interference. The noise in IMU devices usually come from the circuits or the sensors. Eliminating all noises is impossible, so the noise reduction methods are trying to detect and reduce the amplitudes of noises. On the other hand, some noises appear randomly (white noises), and catching

\footnotetext{
${ }^{11}$ Federal communications commission of the USA
} 
them with a single formula is complicated.

For analyzing the gadget accelerometer noises, I place that on five different and static points (like desks) in our lab and recorded the accelerometer sensor data for 20 seconds. I discovered bias noises in the range of $[-0.005-0.01]$ in all axes (see fig. 3.5).

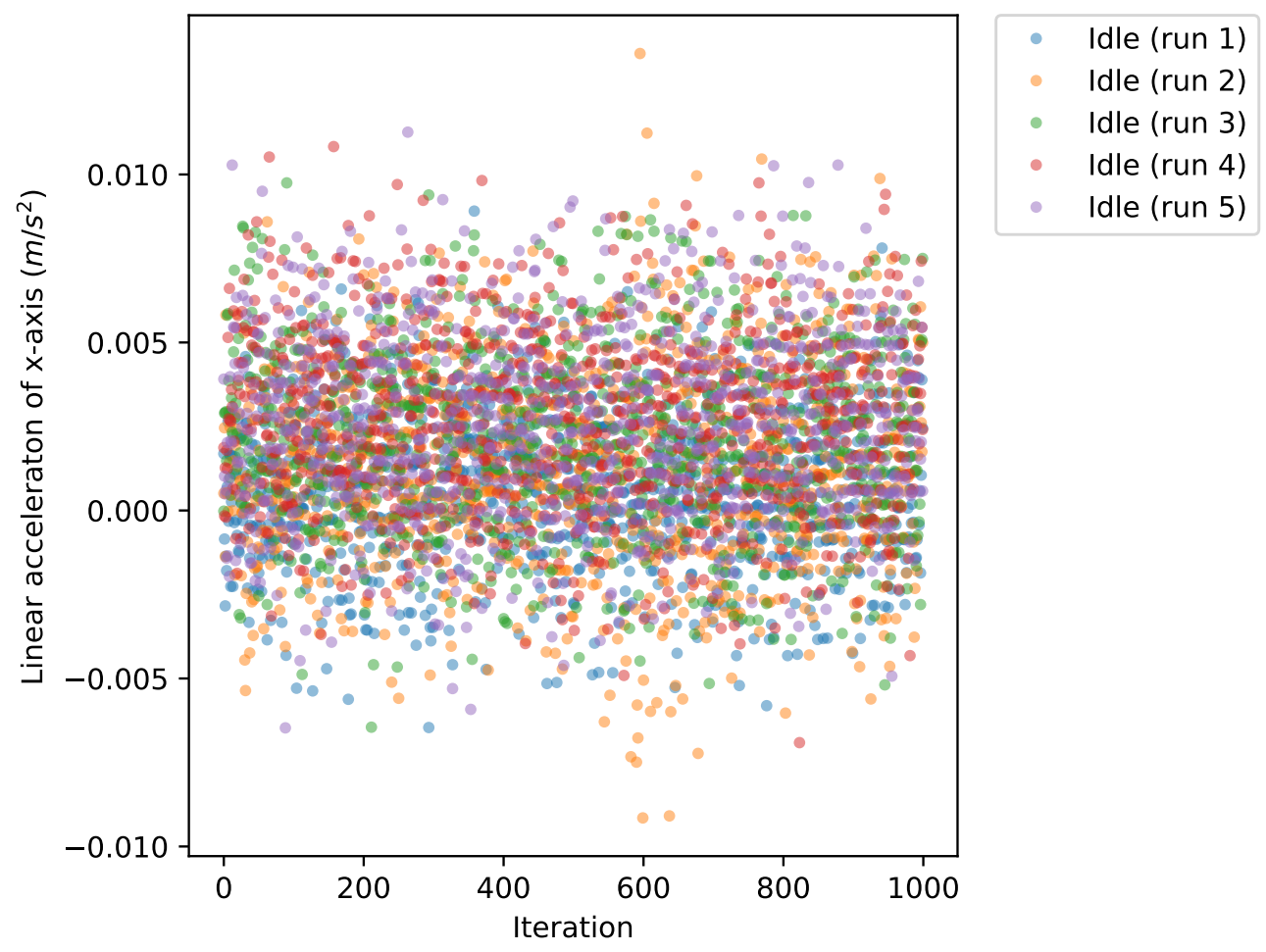

Figure 3.5: Acceleration noises in idle mode $(50 \mathrm{~Hz}, 20$ seconds).

I applied the Fourier transform (FT) function to accelerometer data to find the noise frequencies. I noticed that the result of FT covered all spectrums of frequencies; therefore, we can assume that the acceleration sensor has random noises and tracking all of them with FT function is not practical. The next section explains two conventional noise reduction methods. 


\section{Calibration}

Moving average is a simple low-pass filter and estimates the average series of data points over time to smooth the data fluctuation.

Simple moving average (SMA) is the primary function of this kind, which takes a range of sample (window) of input and returns the mean value of window as output. The mean value shifts forward by adding new data, so this formula always uses fresh new data. In this method, each data point has the same weight regardless of time, and by increasing the window length, the smoothness of the result increases. The second function is exponential moving average (EMA), which uses the same procedure as SMA, but recent data has more weight than historical data. EMA uses a coefficient of $\alpha$, in the range of $[0-1]$ to control the filtering strength.

Equation 3.1 shows the SMA formula notation which $X$ is source, $n$ is length of window and $i$ is index number. Equation 3.2 represents the EMA function notation, and figs. 3.6a and 3.6b represent the result of applying both filters to accelerometer data in idle mode. 


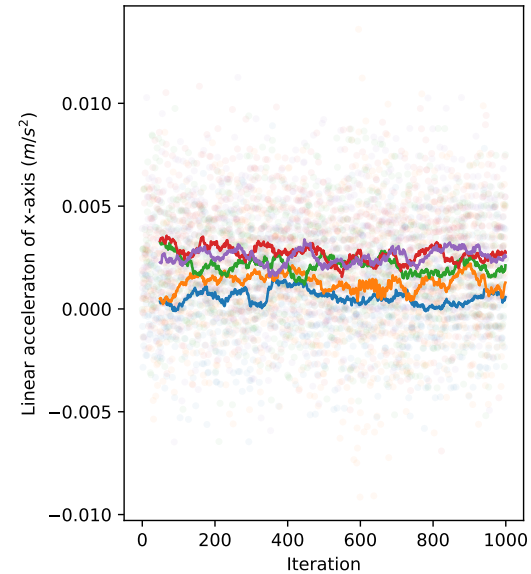

(a)

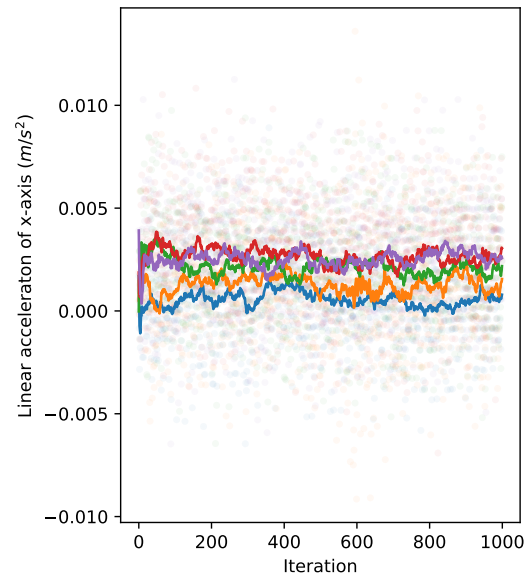

(b)

Figure 3.6: Simple moving average (a) and Exponential moving average (b) filters applied to the acceleration data.

$$
\begin{aligned}
& X_{\text {filter }}=\frac{X_{i}+X_{i-1}+X_{i-2}+\ldots X_{i-(n-1)}}{n} \\
& X_{\text {filter }}=(1-\alpha) * X_{\text {filter-1 }}+\left(\alpha * X_{i}\right) \\
& \alpha \in[0,1]
\end{aligned}
$$

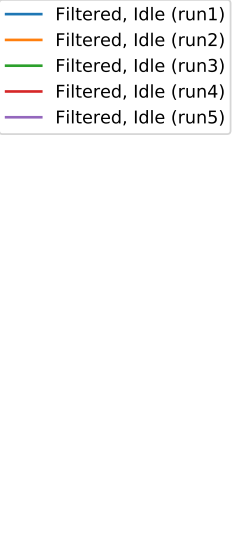




\section{Chapter 4}

\section{Methodology}

This chapter covers methods for collecting, analyzing, and evaluating data. The first section defines research questions and explains the data source, data types and data collection methods. In the second section, two methods for time-series alignment will be explained, and we will review the data clustering function. Finally, three different approaches for evaluating data and a heuristic decision model for making the automatic page-turner will be reviewed. 


\subsection{Research Questions}

An automatic page-turner requires hardware and software components which only the accurate synchronization between them leads to the proper result. The IMU device and acceleration signal properties were described thoroughly in the previous chapter.

After playing a music piece and recording the wrist movements, by assuming that all signal peaks were generated by pressing keys, the trend of spikes can be seen visually. The first question in this research is finding a robust solution to identify signal spikes and create a time series based on signal peak values.

Like previous studies, we need a reference (in form of time-series) to compare to collected data. The second question tries to find an optimum solution for comparing and aligning the mentioned time series. 


\subsection{Data Collection}

In the planning phase of this study, I decided to recruit and invite participants from the music department of Carleton University by posting invitations or posters on message boards. I designed a poster and added the first ten measures of Rondo alla turca by Wolfgang Amadeus Mozart on that and asked students to guess the name of this piece and send it to the dedicated email address of this research (piano@carleton.ca). I thought if someone can sight-read and recall the name of the piece, he/she can probably play that as well.

At the beginning of this research in March 2020, we were exposed to the pandemic of COVID-19, and the Carleton University research committee banned all experiences involving human participation until further notice.

In this study, I played the role of participants and played music pieces several times and in each iteration I tried to change my playing articulations as follows to simulate different and unique playing styles.

- I shifted the tonality of each piece, so every time, different piano keys were used. Generally, all pieces should be played in the same tonality, but this practice helped me playing the same piece in different tonality with various hand movements.

- I also played each run with different fingering. Although the standard fingering in many sheet music is noted, many pianists (beginners specifically) do not follow that completely.

- Finally, I played each part with various dynamics. So I tried to play 
entire or part of pieces with a different dynamic.

I selected five piano pieces (see table 4.1) that were easy to play for beginners and intermediate piano players. After searching and downloading MIDI files of selected piece, I used MuseScore ${ }^{1}$ application in Windows 10 and shifted all pieces to the nearest major and minor tonality. So for each piece, I had three different tonalities.

\begin{tabular}{llcc}
\hline Piece Name & Composer & Duration (s) & Temp (bpm) \\
\hline Adagio in G minor & Tomaso Albinoni & 100 & 62 \\
Golden Dreams & Javad Maroof & 170 & 100 \\
Rondo Alla Turca & W.A Mozart & 60 & 90 \\
Sonatina Op. 20 & Jan Ladislav Dussek & 134 & 120 \\
Twenty One Pilots & Chlorine & 70 & 90 \\
\hline
\end{tabular}

Table 4.1: Details of played music pieces in experimental sessions.

For tracking the hand motions, I used a pair of NGIMU kits, as described in section 3.2.1. This device has a graphical user interface (GUI) for managing, monitoring and capturing data. NGIMU offers the access point (AP) and client mode (CM) for wireless connection. By setting device in AP mode, the built-in WIFI adapter acts as a router, so other devices like laptops can connect to NGIMU module. AP mode creates a $\mathrm{P}^{2} \mathrm{P}^{2}$ connection, computers read data from the gadget directly.

In the CM, NGIMU should be connected to an external WIFI router. For this purpose, first, we must connect NGIMU with a USB cable to the computer and set the WIFI router credentials on the NGIMU setting page. In

\footnotetext{
${ }^{1}$ MuseScore is music score-writing software. This application is multi-platform and can be run in Windows, macOS, and Linux operating systems. It is released as free and open-source software under the GNU General Public License.

${ }^{2}$ Peer to peer
} 
this mode, the device first tries to connect to the WIFI router and then continuously sends data to a specific IP address. I described the WIFI settings that I used for this study in table 4.2.

\begin{tabular}{|c|c|c|c|}
\hline \multicolumn{1}{c|}{} & Property & Description & Value \\
\cline { 2 - 4 } Client & SSID & Shared WIFI router name & BELL6500 \\
\cline { 2 - 4 } & KEY & The WIFI Router password & * $* * * * * * * *$ \\
\cline { 2 - 4 } & IP Address & $\begin{array}{c}\text { Using Dynamic Host } \\
\text { Configuration Protocol }\end{array}$ & True \\
\hline \multirow{4}{*}{ UDP } & Send IP Address & $\begin{array}{c}\text { Main comput IP Address } \\
\text { for sending data }\end{array}$ & 192.168 .2 .27 \\
\cline { 2 - 4 } & Send Port & $\begin{array}{c}\text { Computer's open port for } \\
\text { sending }\end{array}$ & 8200 \\
\cline { 2 - 4 } & Receive Port & $\begin{array}{c}\text { Module's open port for } \\
\text { receiving data }\end{array}$ & 1020 \\
\hline
\end{tabular}

Table 4.2: Default Configuration of NGIMU device for this study

Most personal computers only have one wireless card, so the parallel connections to two access points concurrently with one card is not achievable. In this study, I had to record data from both gadgets simultaneously, using the AP mode for transmitting data was not practical. I used client mode, and both devices sent data at the same time to the main computer.

The NGIMU kit can send its sensor data up to $400 \mathrm{~Hz}$. This kit also has a built-in AHRS fusion algorithm to measure the kit orientation relative to the Earth as a quaternion, rotation matrix, or Euler angles. I have set the $200 \mathrm{~Hz}$ as a sampling frequency for sensors. The accelerometer, gyroscope, and magnetometer sensors send data for $x, y, z$ axes separately.

In previous sections, I extensively used three-dimensional axes term. It is needed to explain the direction of those axes in the environment of my study. All hand movements (left or right) alongside the piano keys are defined as 
$y$-axis. The first piano key $(A)$ represents $-y$, and the last key on the right side $(C)$ is $+y$. Other movements from white keys to black keys and vice versa are on the $x$ axis. The black keys area is defined as $+x$, and the white keys are at $-x$. Finally, all up and down movements are mapped to the $z$ axis. By defining the piano key surface as 0 , the upper area is $+z$, and each pressing key generates data in $-z$ (see fig. 4.1).

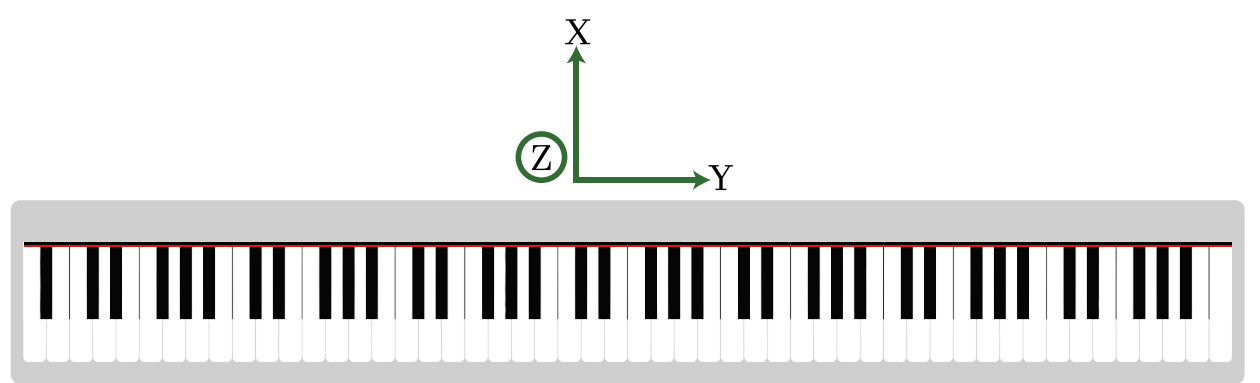

Figure 4.1: The three-dimensional axes reference in this study.

The first challenge that I faced in collecting data was syncing both hands together. In many piano pieces, both hands do not necessarily start playing simultaneously. On the other hand, the internal timestamp of gadgets resets every time and is not reliable. Therefore, finding start points and syncing both hands was not possible. To overcome this problem, I defined the wristtwisting (WT) gesture (supination and pronation). The recording process starts by pressing the record button on both data-logger windows; then, users move their hands over the piano keys at the place that they want to start to play. At the beginning and the end of the play, users must supinate their wrist $180^{\circ}$ (for the right hand, this rotation is clockwise and for the left hand is counterclockwise) and then return both wrists to the normal position and start playing. This gesture creates significant spikes in the gyroscope signal 
(on $x$-axis), so the system can find both start and endpoint of the recording by detecting those peaks (see fig. 4.2).

Finding signal peaks and extracting data between them can be done in following steps:

- Normalizing gyroscope data in range of $[-1,1]$

- Finding the first minimum value from the first half of the gyroscope signal.

- Finding the next point that has the closest value to the mean value (Start point)

- Finding the last maximum value from the other half of the gyroscope signal

- Finding the previous point that has the closest value to the mean value (End point)

- Collecting the data between start and end points.

I set the same frequency of all sensors, so the number of recorded data was the same. So, the range of valid data extracted by WT-gesture is also valid for accelerometer data. 


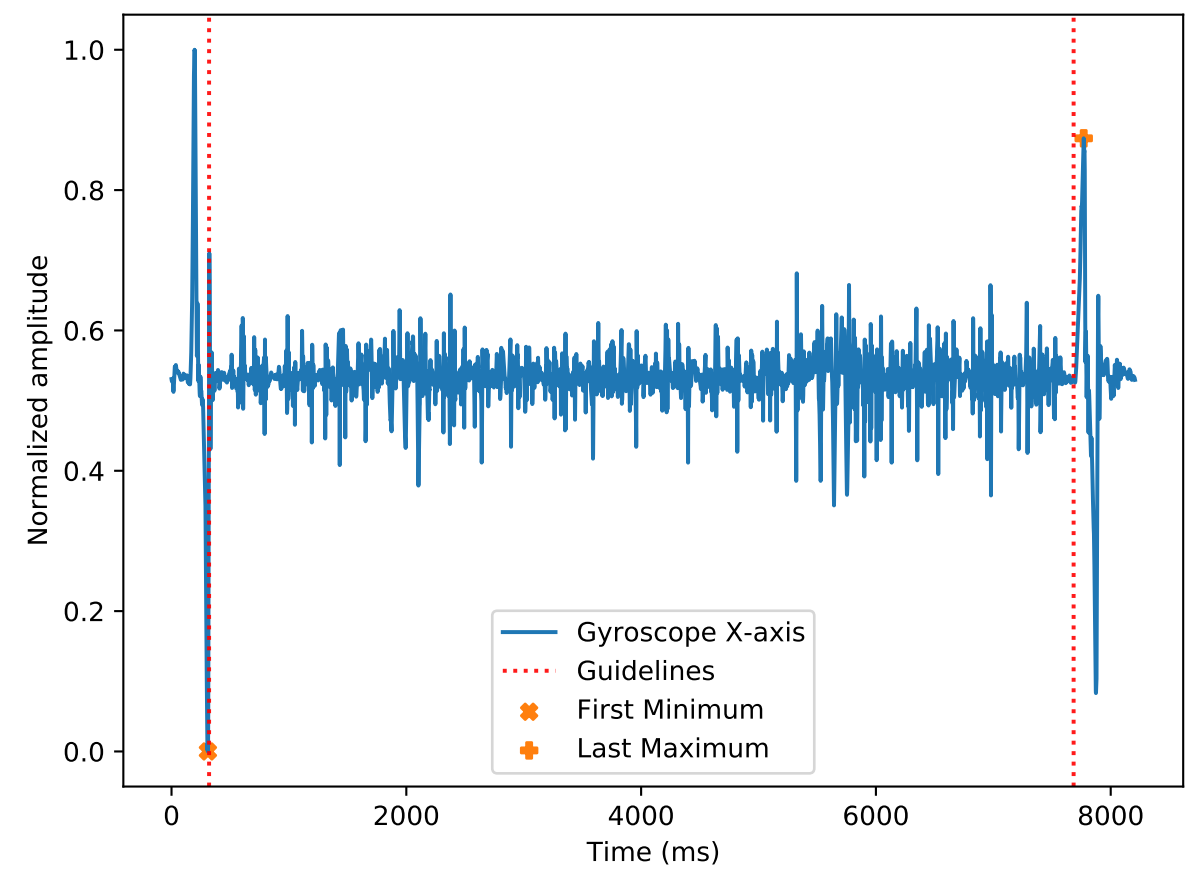

Figure 4.2: The wrist-twisting gesture detector detected the boundaries of valid data. 


\subsection{Data Analysis}

\subsubsection{Time Series Alignment}

\section{Dynamic Time Warping}

Dynamic time warping (DTW) algorithm attempts to determine the best global alignment between two data sequences or time series based on data point distances. This method is widely used in various disciplines, such as speech recognition[50, 51], data clustering[52, 53], gesture detection[54, 55, $56,57]$, and also in the field of this study $[3,58,59]$.

For $x, y$ series as $x=x_{1}, x_{2}, \ldots . x_{n}$ and $y=y_{1}, y_{2}, \ldots . y_{n}$ the local distance for each pair is denoted as $d(x, y)=\left|x_{i}-y_{j}\right|$ which $i, j$ are indexes. DTW algorithm uses $(m \times n)$ matrix (distance matrix) for measuring optimum distance, which $m, n$ are the length of the first and second series. In this matrix, the cell values are filled by of the three equations in eq. (4.1). The calculation process to find the optimum alignment between pairs begins with the last cell (top-right) and ends with the first cell (bottom-left). So, the first and last elements of this collection are paired with each other. Next, on each iteration, the minimum value of neighbour cells is selected, and this process continues to the first cell. The combination of the selected cells defines the optimum (minimum distance) of alignments.

Fig 4.4 shows the result of DTW function applied on two vectors $(x=$ $[1,3,1,2,1,1,1], y=[1,1,3,1,2,1,1])$. The values in the middle of the graph represent the local distances and the blue line shows the optimal path $(W)$. 


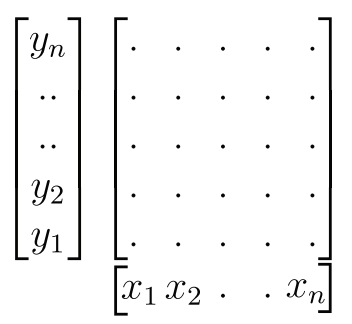

Figure 4.3: Cost matrix schema for solving DTW problems.

D for the first column $\left(x_{1}\right)=\left|x_{i}-y_{i}\right|+D\left[x_{-1}, 0\right]$

D for the first row $\left(y_{1}\right)=\left|x_{i}-y_{i}\right|+D\left[0, x_{-1}\right]$

D for other cells $=\left|x_{i}-y_{i}\right|+\min \left\{\begin{array}{c}D\left[x_{-1}, y_{-1}\right] \\ D\left[x_{-1}, y\right] \\ D\left[x, y_{-1}\right]\end{array}\right\}$
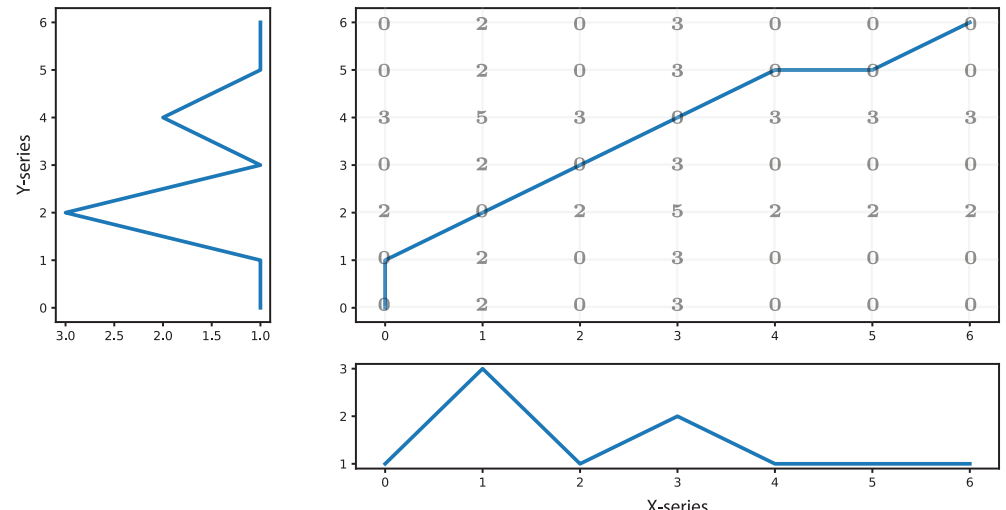

Figure 4.4: The results of DTW function on two series. The digits inside the matrix show the local distances and the blue line indicates the optimum path.

For solving problems with DTW algorithm, the following rules should be considered:

- Items from the first series aligns with one or more nodes in the second series. 
- The first and last items of the first series must be aligned with their equivalents in the seconds series, respectively. These nodes also can have more alignments.

- The $W$ (optimum path) is continuous and monotonic. Therefore, the alignment indices always increase and do not go back.

\section{Cross Correlation}

Cross-correlation (CC) is one of the most common algorithms of signal processing that measures the similarities of two signals when a function of a time-lag applied to one of them. Many disciplines like radar systems, speech recognition use this method for finding similar patterns in signals or dataset. The discrete mathematical function of cross-correlation of $f$ and $g$ is:

$$
(f \star g)[n] \triangleq \sum_{m=-\infty}^{\infty} \overline{f[m]} g[m+n]
$$

The process of calculation starts by fixing the first series as a reference. In a simple loop, the $\mathrm{CC}$ function applies a range of $[-a,+a]$ values to all data points of the second series. In each iteration, aligned items in both series should be multiplied, and the sum of them indicates the correlation for series for that specific value (lag). The maximum value of all iterations determines the best similarity between series.

All steps of CC function for two signals as: $f=[8,9,11,12,8,9,11,13]$ and $g=[5,6,2,3,4,6,7,4]$ with the range of lags between $[-1,2]$ is shown in Table 4.3, and visualized in fig. 4.5. 


\begin{tabular}{l|l|l} 
Lag & \multicolumn{1}{|c|}{ Calculation } & Result \\
\hline-1 & $(f \star g)=(8 \cdot 6)+(9 \cdot 2)+(11 \cdot 3)+(12 \cdot 4)+(8 \cdot 6)+(9 \cdot 7)+(11 \cdot 4)$ & 302 \\
0 & $(f \star g)=(8 \cdot 5)+(9 \cdot 6)+(11 \cdot 2)+(12 \cdot 3)+(8 \cdot 4)+(9 \cdot 6)+(11 \cdot 7)+(13 \cdot 4)$ & 367 \\
1 & $(f \star g)=(9 \cdot 5)+(11 \cdot 6)+(12 \cdot 2)+(8 \cdot 3)+(9 \cdot 4)+(11 \cdot 6)+(13 \cdot 7)$ & 352 \\
2 & $(f \star g)=(11 \cdot 5)+(12 \cdot 6)+(8 \cdot 2)+(9 \cdot 3)+(11 \cdot 4)+(13 \cdot 6)$ & 292
\end{tabular}

Table 4.3: The steps of applying cross-correlation function to $f, g$ series.
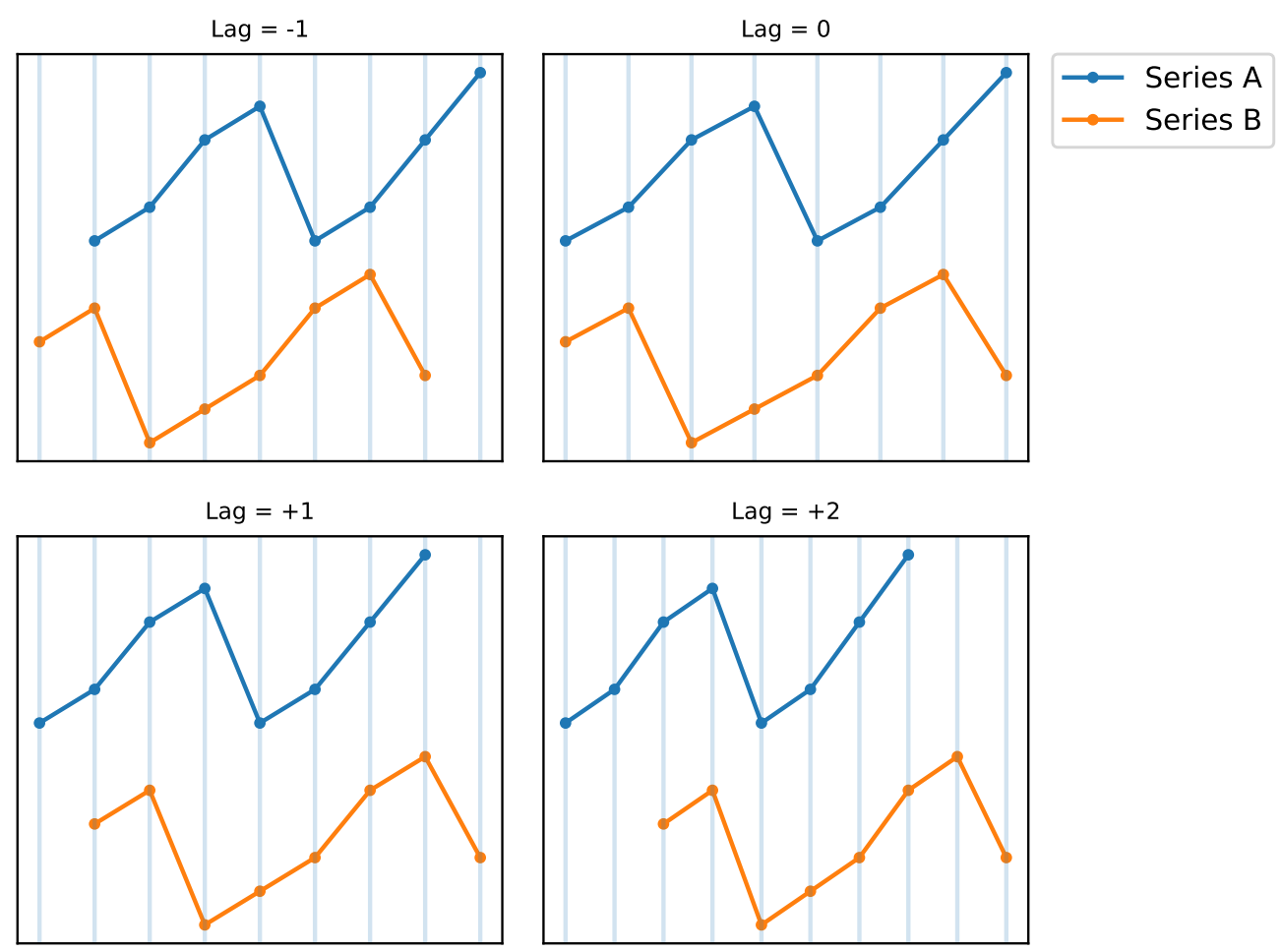

Figure 4.5: Cross-correlation function applied to series A,B with the range of lags $[-1,+2]$. 


\subsubsection{Data Clustering}

Data clustering is a primary topic in machine learning field, aims to categorize data in several partitions based on their similarities. I used K-means data clustering methods in the decision model of this study. This section explains two types of machine learning approaches and K-means method in detail.

\section{Machine Learning Methods}

In general, we can categorize machine learning algorithms into three main models as supervised, unsupervised and reinforcement learning. The supervised model works with trained data, which means researchers have already labelled historical data (inputs) with correct answers or values (outputs). The valid supervised model predict the correct label of new inputs independently. This method is ideal for classification problems such as binary or multiclass, ensembling, and regression modelling. This approach requires direct supervision, especially during the data collection phase, and needs continuous rebuilding and updating the model to ensure valid outcomes. Supervised methods provide reliable results, but developing models are time-consuming, complicated, and need computation loads.

The structure of the unsupervised approach is the opposite. This method works with unlabelled data and attempts to identify patterns for labelling data without supervision. This approach is suitable for clustering, association mining, and anomaly detection problems. Unsupervised method outcomes are more unpredictable than supervised; however, this method gives a wide range of options for solving complex problems. 


\section{K-means}

K-means is an unsupervised clustering method that seeks to partition data in a specific number of clusters $(k)$. So, the data points in each cluster have more similarities with each other rather to points in other clusters. The following steps show the steps of solving a problem with K-means algorithm. I visualized 5 iterations of K-means method for a random dataset with $k=3$ in fig. 4.6.

1. Determining the number of clusters to identify $(k)$.

2. Placing random and distinct (non-overlapped) data points as the initial centers for each cluster.

3. Calculating Euclidean distance of each point to clusters center point. The distance between two points in two-dimensional space is denoted by $d=\left(\left(x^{1}-x^{2}\right)-\left(y^{1}-y^{2}\right)\right)^{\frac{1}{2}}$

4. Assigning each point to the nearest cluster center.

5. Calculating the mean for each cluster to get a new centroid.

6. Going back to step 3 until there are no changes in clusters center. 


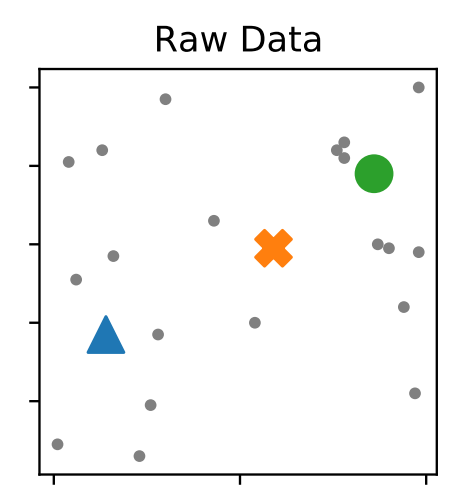

Iteration 3

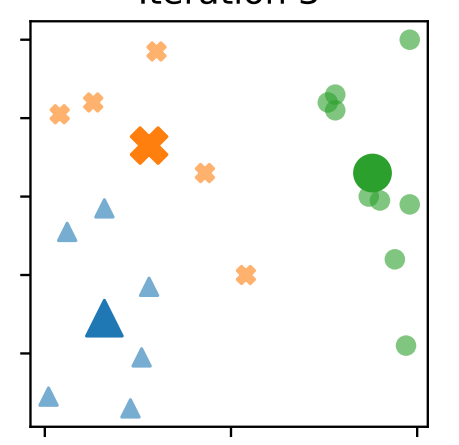

Iteration 1

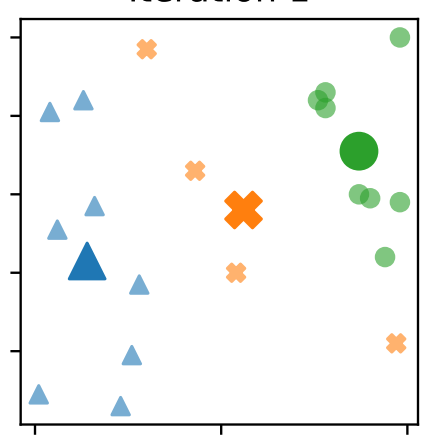

Iteration 4

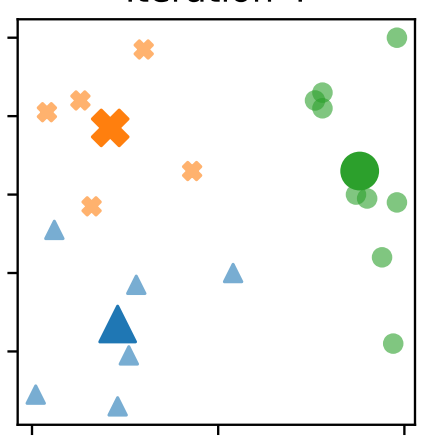

Iteration 2

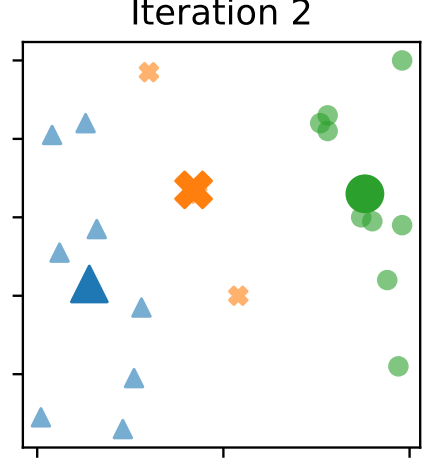

Iteration 5

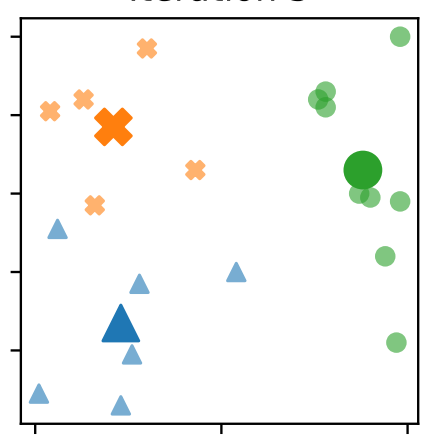

Figure 4.6: Five iterations of K-means clustering function for a dataset when $k=3$. Each symbol represents the cluster centroids and the lighter symbols represent the members of each cluster. 


\subsubsection{Signal Peaks Finding}

Pressing the piano keys requires the hand movement from the top to bottom (along the $z$ axis). The acceleration and deacceleration of this act generate spikes in acceleration signal. I have already discussed this phenomenon in section 3.2.2.

Peak detection is another fundamental topic in the field of signal processing. Several techniques such as using window-threshold[60], wavelet transform[61], and some advanced methods with complex algorithms such as Kalman-filter and Hidden Markov model[62, 63] are proposed in literature. In this study, I developed two semi-heuristic approaches for finding peaks in accelerometer signal. In the next part, I will explain both briefly.

\section{K-means Approach}

Musicians have their own style, and their playing articulations for particular notes can be different. These differences can affect the velocity and the pressure that they use for pressing piano keys. For designing a dynamic tool for detecting signal peaks, I used K-means method (see section 4.3.2). Like other signal processing functions, we should do some data preparation, which I will explain in the next sections. For explaining this section, it is necessary to consider that I will remove negative values from accelerometer data, so the range of valid data for implementing K-means would be [0 1]. By this comment, applying K-means with $k=2$ returns two clusters. The first cluster has data points with high amplitudes which is a suitable candidate for having signal peaks, and the second one contains values with 
low amplitudes.

\section{Geometric Angle Approach}

My observation showed the signal spikes resulting from pressing piano keys were significantly sharper than other peaks (when my hand was moving over piano keys or was in idle mode). I came up with the idea of calculating the geometric angle of spikes for detecting peaks. Assuming the peak is one angle of a right triangle. The angle degree can be obtained with Law of cosines $\left(b^{2}=a^{2}+c^{2}-2 a c \cdot \cos (\beta)\right)$.

I compared K-means and Geometric-angle clustering approaches in fig. 4.7. 

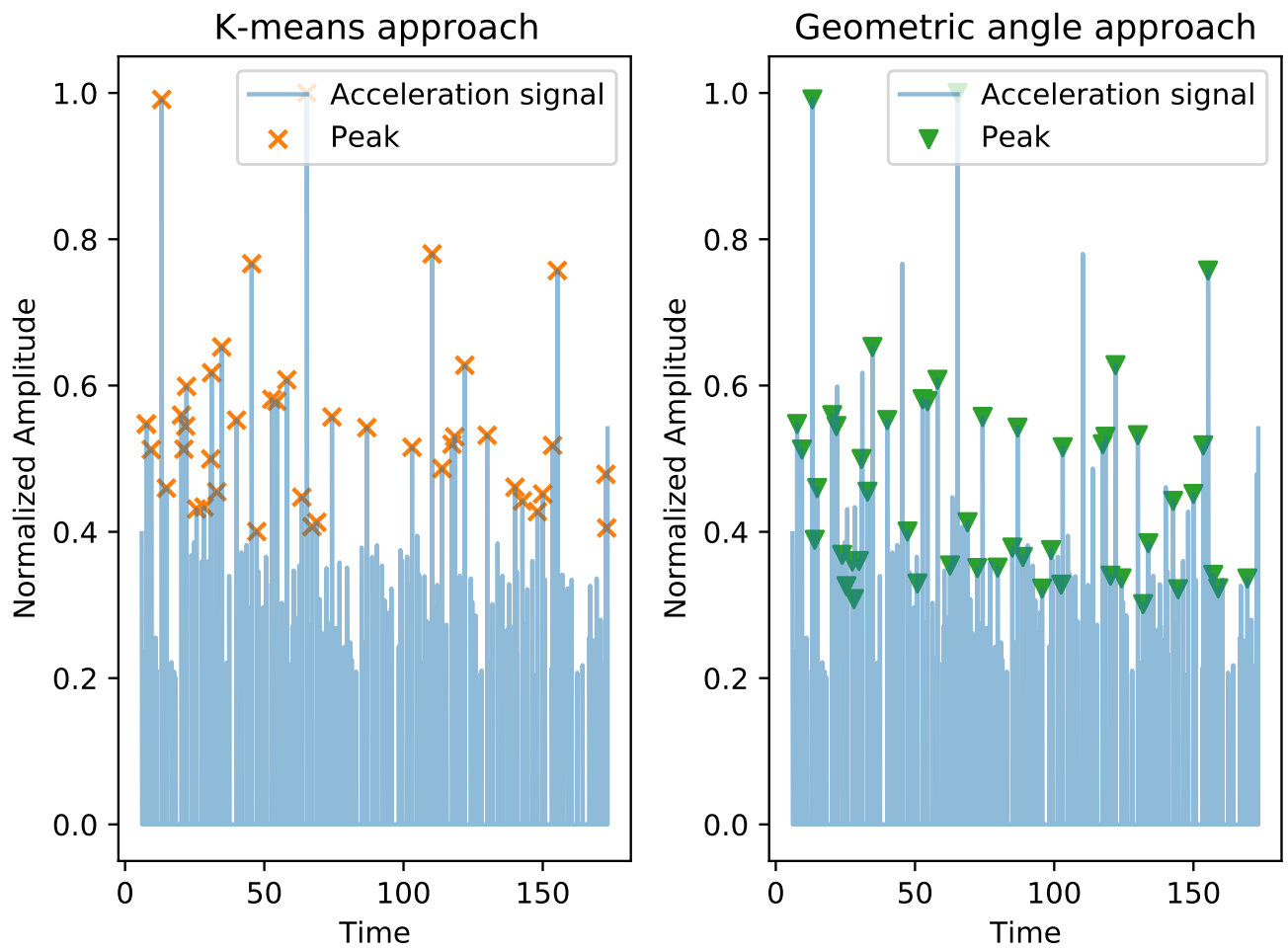

Figure 4.7: Shows signal peak clustering with K-means algorithm (Left) and Geometric angle (Right with angle $<5^{\circ}$ ). 


\subsection{Evaluation}

This section explains the evaluation process of the collected data. Then, three different approaches for labelling data and measuring errors in this system will be described. Finally, I will explain the heuristic decision model that I developed to evaluate several criteria and decide whether to turn the music page automatically.

\subsubsection{Alignment Types}

In time-series alignment section (4.3.1), we learned how the dynamic time warping can find the optimum alignment between time series. In this study, three different terms are suggested for categorizing the possible alignments between series (see fig. 4.8).

- Aligned: happens when one node from the first series is aligned with one node in the second series, the value of both nodes is equal, so there is no time latency between aligned pairs. This type has the highest value among other types of alignments. I used the " $\longleftrightarrow$ " symbol to indicate this type of alignment in the result chapter.

- One-to-one: similar to the aligned type, but the values of nodes are

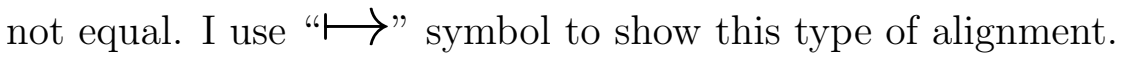

- One-to-many: A node from the first series is coonected to several nodes from the other series (and vice versa), this happens when the length of the series is not equal. I used "ד" symbol to show this type of connection. 


\begin{tabular}{|c|c|c|c|c|c|c|}
\hline i & i & $\begin{array}{l} \\
1 \\
1 \\
1 \\
1 \\
1 \\
1 \\
1 \\
1 \\
1 \\
1\end{array}$ & $\begin{array}{l} \\
1 \\
1 \\
1 \\
1 \\
1 \\
1 \\
1 \\
1 \\
1 \\
0\end{array}$ & $\begin{array}{l}3 \\
I \\
I \\
I \\
I \\
I \\
I \\
1\end{array}$ & 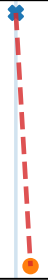 & $\begin{array}{l} \\
\text { III } \\
\text { II } \\
\text { IIII } \\
\text { III } \\
\text { III } \\
\text { III } \\
\text { IIII } \\
\text { III } \\
\end{array}$ \\
\hline
\end{tabular}

Figure 4.8: DTW alignment types.

\subsubsection{Error Metrics}

In this study, error refers to the numerical distances between observed data (time series of pressed keys) and the actual data (time series of digital notes extracted from MIDI file). Generally, time and value are attributes of timeseries elements. At this point, only the time sequences of series should be compared, so I replaced the data attribute of each series with a constant number. Therefore, the series can be scattered in 2D space. Several academic papers[64, 65] compared error metrics; however, no decisive method was proposed for all cases. This section describes three methods for measuring distances between observed and actual data that returned the acceptable results during the data analysis phase in this research.

\section{Euclidean Distance}

In the Cartesian coordinate system, the minimum distance between two points as $v=\left(x_{1}, y_{1}\right), u=\left(x_{2}, y_{2}\right)$ is equal to the length of straight line between them. We can assume that straight line is a hypotenuse of a right triangle; hence, the Pythagorean theorem can measure its length. The Euclidean distance for two points is denoted in equation 4.2.a and visualized 
in fig. 5.8. Euclidean distance measuring method can be expanded to more dimensions by adding related values to the general equation (see 4.2.b).

$$
\begin{gathered}
D\left(v_{i}, u_{i}\right)=\left(\left(v_{x i}-u_{x i}\right)^{2}+\left(v_{y i}-u_{y i}\right)^{2}\right)^{\frac{1}{2}} \\
D\left(v_{i}, u_{i}, . ., z_{i}\right)=\left(\left(v_{x i}-u_{x i}\right)^{2}+\left(v_{y i}-u_{y i}\right)^{2}+. .+\left(v_{z i}-u_{z i}\right)^{2}\right)^{\frac{1}{2}}
\end{gathered}
$$

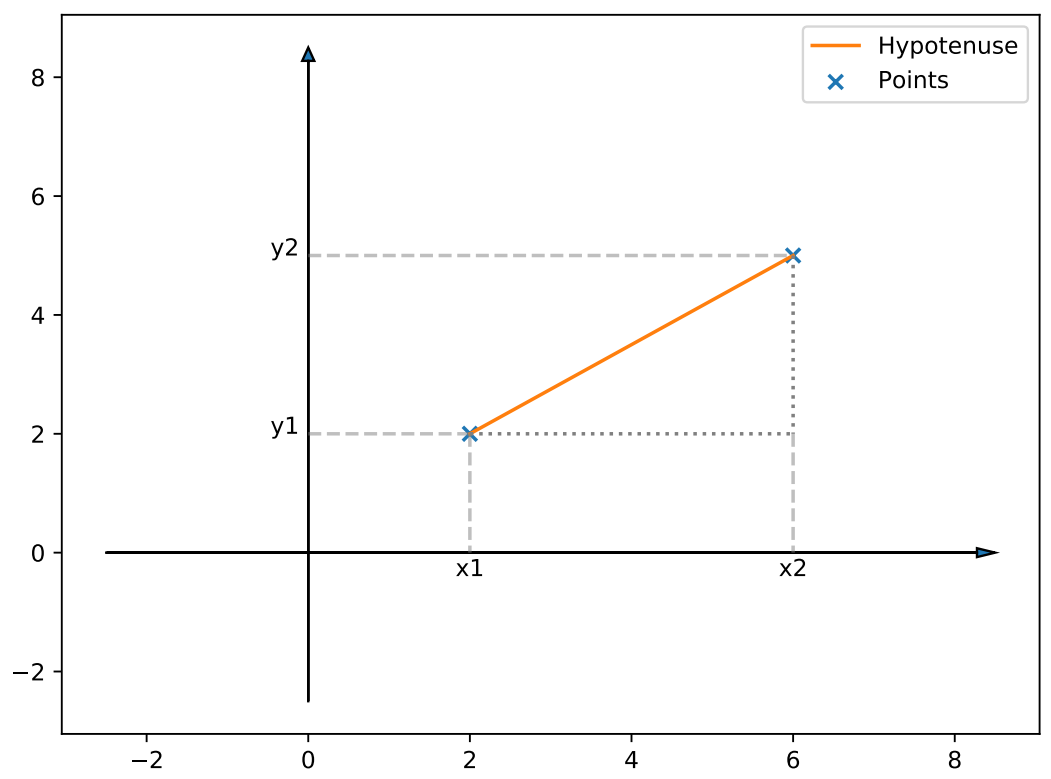

Figure 4.9: Straight line between two points can be considered as a hypotenuse of a right triangle, and its length can be obtained by Pythagorean theorem.

\section{City-block Distance}

City-block or Manhattan distance is another method for measuring distances between two points in two-dimensional space. Unlike the Euclidean distance, which used the power to keep the differences positive, this formula uses the absolute values. In other words, instead of calculating the hypotenuse of the 
imaginary triangle, the sum of the length of two different sides of hypothetical triangle returns the distance of two points (see equation 4.3 and fig. 4.11).

$$
D\left(v_{i}, u_{i}\right)=\left|v_{x i}-u_{x i}\right|+\left|v_{y i}-u_{y i}\right|
$$

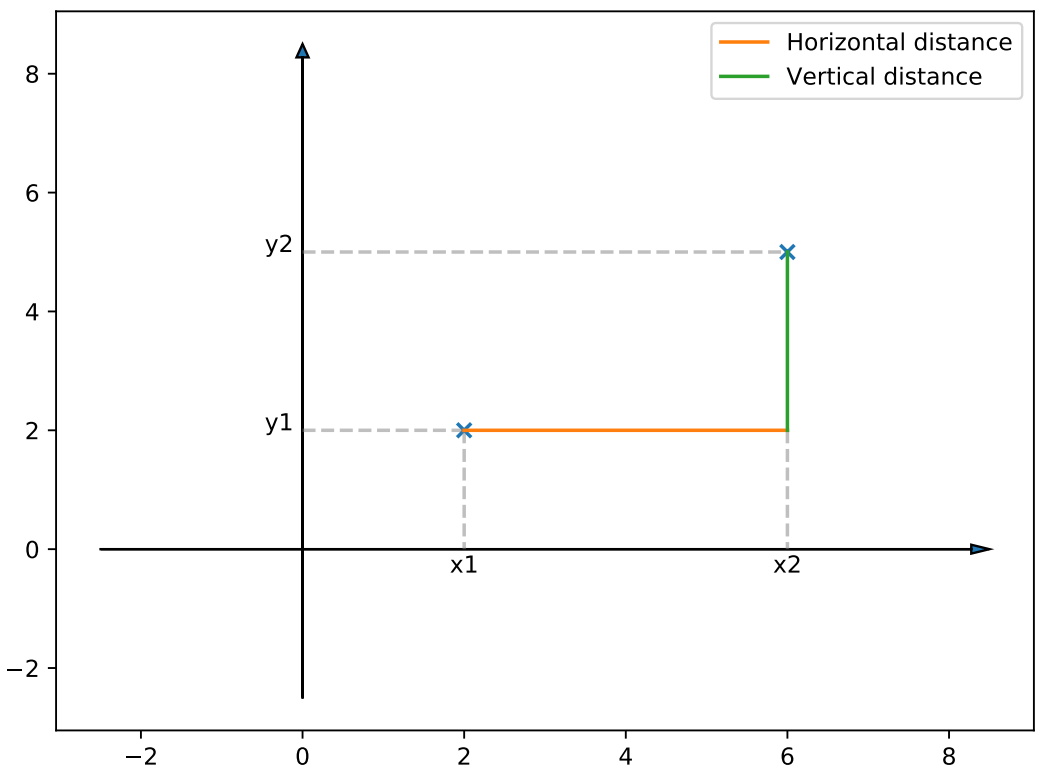

Figure 4.10: Shows the Manhattan distance sample in which the distance of two points is equal to the length of horizontal and vertical distances.

\section{Minkowski \& Cosine Distance}

The Minkowski distance is the generalized method of Euclidean and Manhattan distance methods. The mathematical formula is denoted in Equation 4.4. By setting $p=1$ and $p=2$, the results are equal to Manhattan and Euclidean distance respectively.

$$
D\left(v_{i}, u_{i}\right)=\left(\left|v_{x i}-u_{x i}\right|^{p}+\left|v_{y i}-u_{y i}\right|^{p}\right)^{\frac{1}{p}}
$$


Cosine distance uses a trigonometric approach for measuring distance of two points. In two-dimensional space, all possible positions of two points can always be fit into a circle area, so the range of results in this approach is between $[-1,1]$.

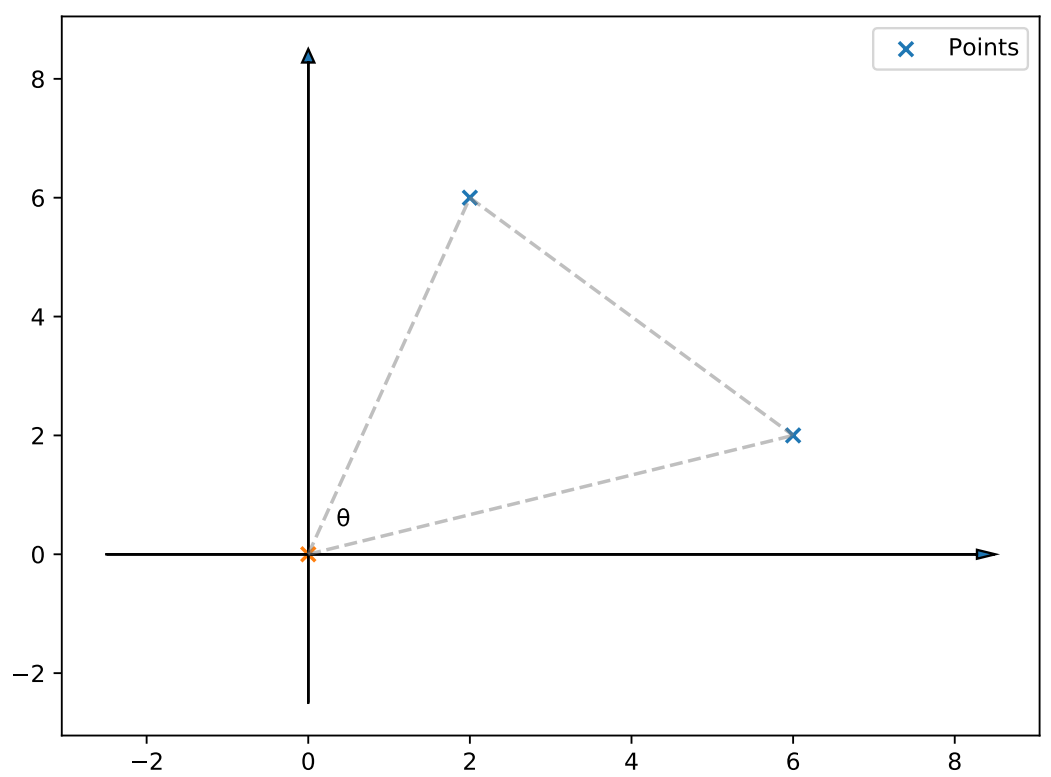

Figure 4.11: Shows points in two-dimensional space. The cosine of $\theta$ determines the relative distance between points.

\subsubsection{Measure Labelling}

In the music theory section (2.1), I explained the concept of measures in music notation. I discussed their importance as suitable units for dividing a piece of music into equal chunks. While analyzing the experimental sessions' data, I realized that we could have three probable combinations for every measure of music. 


\section{Type 1}

In the first type, the number of aligned nodes in both series is almost equal. We expect to have all three types of connections (one-to-one, aligned, and one-to-many) in all chunks. This type shows that the pianist played chunks completely (see fig. 4.12), and I marked this chunk as passed.

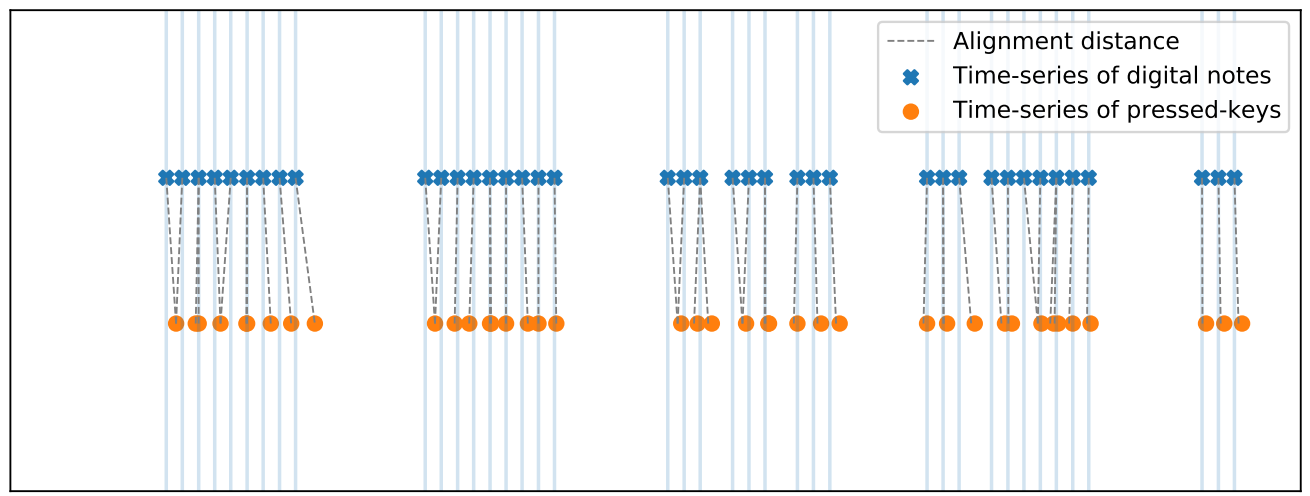

Figure 4.12: Show the first type of chunk alignment, the pianist played all notes.

\section{Type 2}

In this type, most or all notes of a chunk have not been played by the pianist.

So half of the notes are connected to the last time series of pressed keys (TP) node in the previous chunk, and the other half are connected to the next TP node in the next chunk. The distance of this chunk is significantly higher than the previous type. I labelled this chunk as failed. 


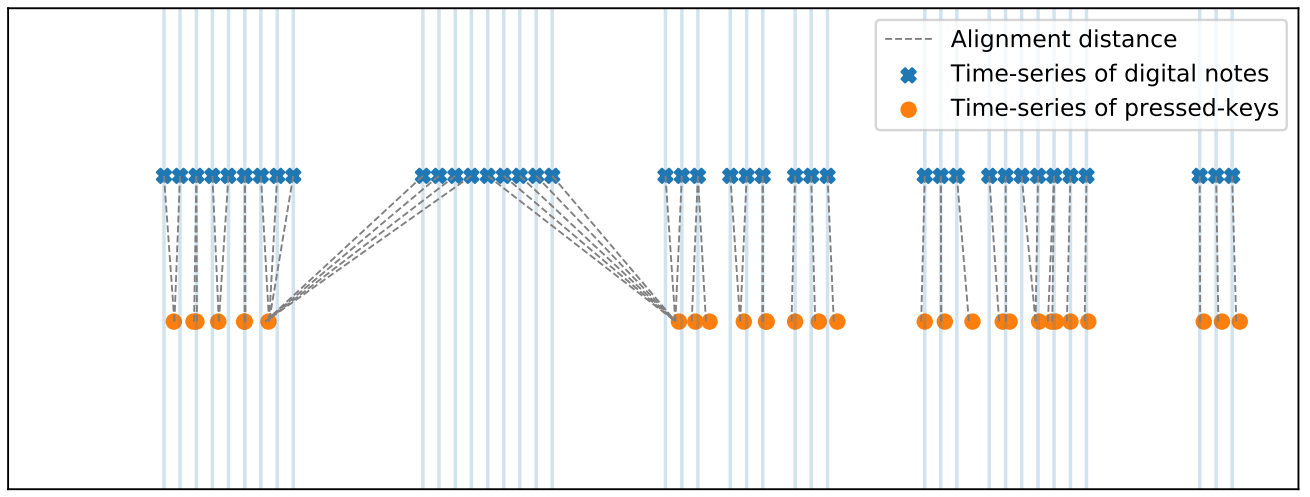

Figure 4.13: The second type of chunk alignment, pianist did not play most or all notes of the chunk.

\section{Type 3}

In this type, the pianist played some notes that should not be played. Half of the TP elements are connected to the last note of the previous chunk, and the other half are connected to the next note of the next chunk. Like the previous type, the distance of this chunk is much higher than the first type. I labelled this chunk as failed too.

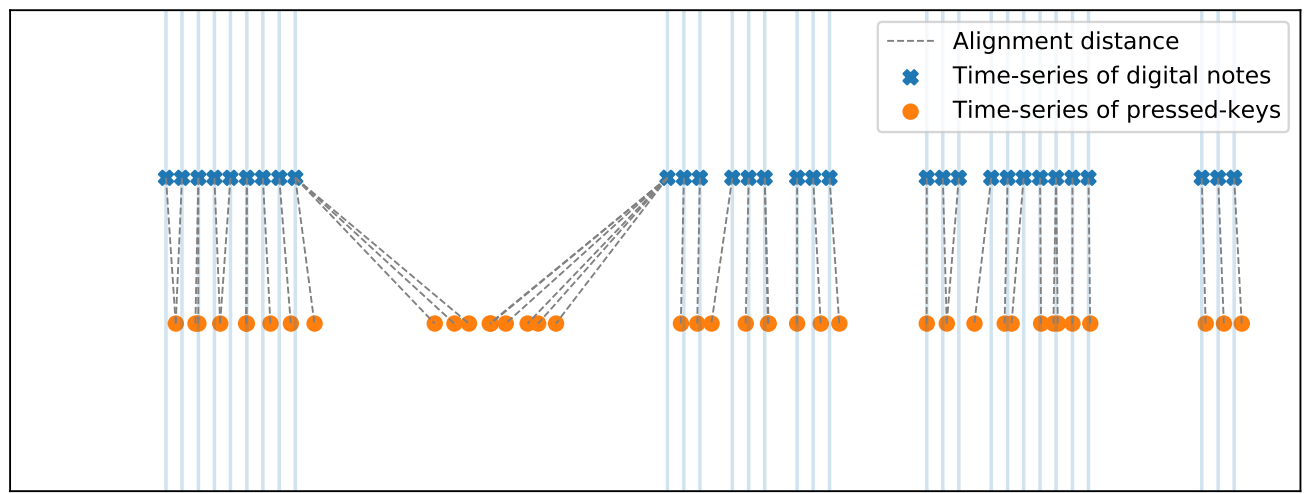

Figure 4.14: The third type of chunk alignment, pianist played extra notes. 


\subsubsection{Decision Model}

In my proposed design, the event of page-turning can be set on any measure/chunk in the music sheet. This part explains the heuristics evaluation method that I developed to decide to execute the page-turning command. The fig. 4.15 shows the flowchart for the decision model. It should be noted that all the numbers and criteria in this section are based on the experiments performed during the study.

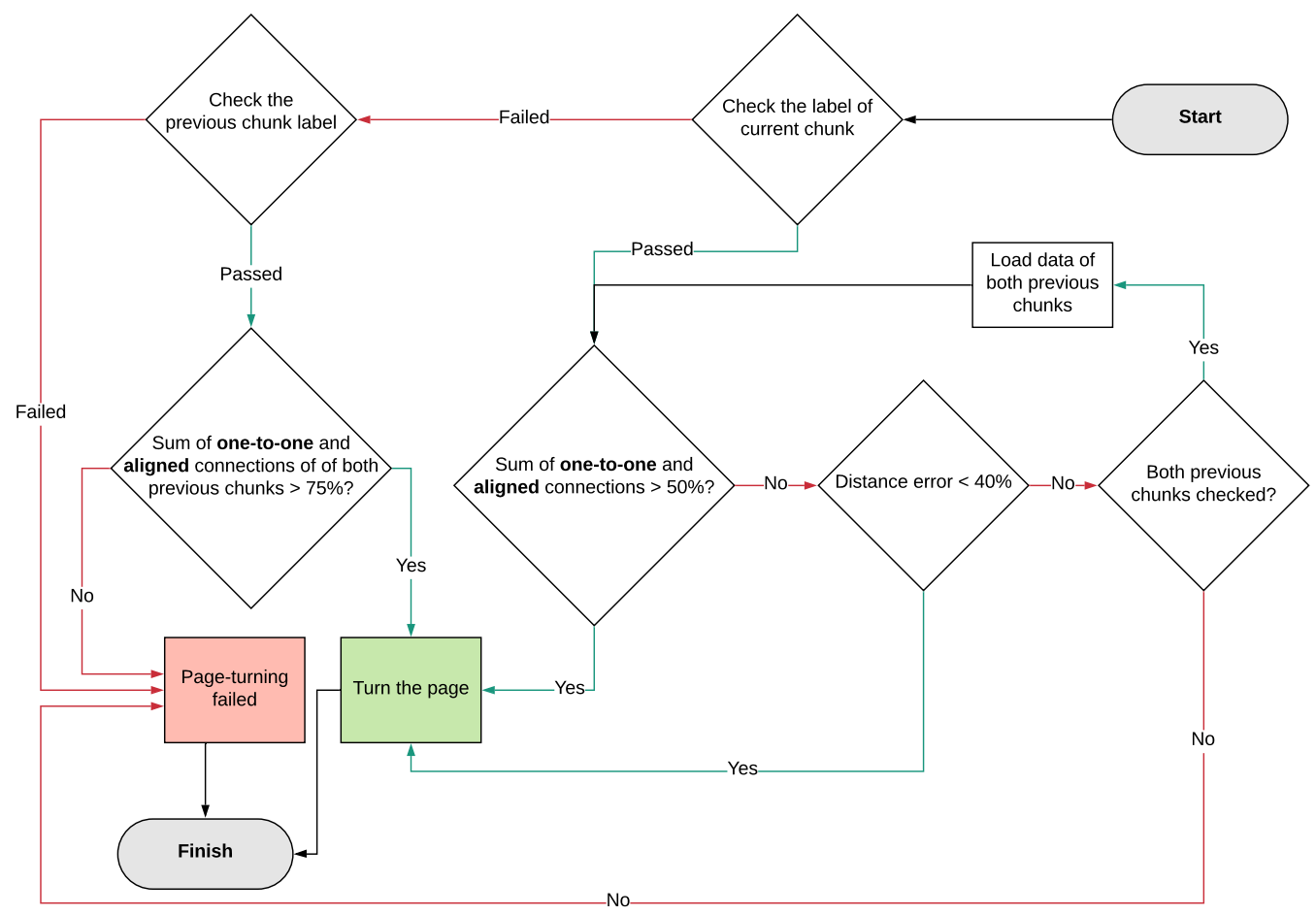

Figure 4.15: Decision model flowchart.

The decision process starts by checking the label of the defined measure to trigger the page-turning event. If the chunk got the failed label, system checks the last two chunks. If any of them has failed labels, system can not detect the current chunk, and the page-turning process ends with failed 
status. However, if the sum of one-to-one and aligned connections of two previous chunks are more than $75 \%$, system remarks chunk to passed and turns the page.

If the nominated chunk has passed label, the process continues to check the sum value of its one-to-one and aligned connections. If this value is more than $50 \%$, it means more than half of the notes have played by the pianist. So, the system accepts the nominated chunk as accurate and turns the page. In case that the sum is less than $50 \%$, system checks the alignment distance error. If the error is less than $40 \%$, system passes this step and turns the page. Otherwise, system checks the two previous chunks to see whether their sum of the one-to-one and aligned connections are more than $50 \%$. If yes, we can accept this chunk as accurate, and system can turn the page; if not, system can not detect the right chunk and ends with failed status. 


\section{Chapter 5}

\section{Tuning the Automatic}

\section{Page-turner}

This section describes all steps of tuning and developing an automatic pageturner system. First, I analyzed all data after each experiment, which helped me better understand all components' characteristics. Eventually, I modified some parts in code to works in a real-time mode (online). Fig 5.1 shows the workflow of experimental study. 


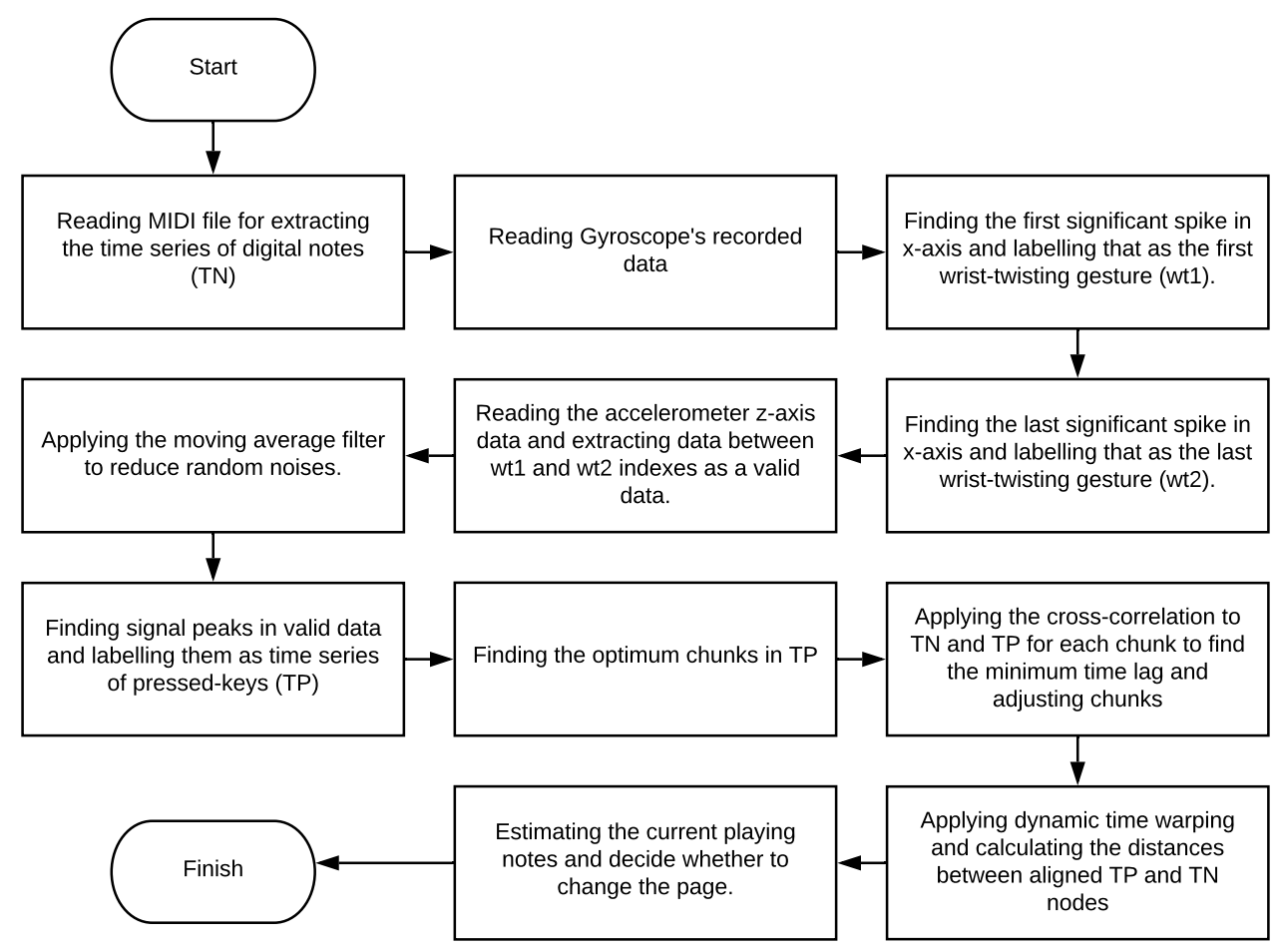

Figure 5.1: Workflow of this study in experimental sessions. 


\subsection{Time Series of Digital Notes (TN)}

As stated in the previous sections, the primary goal of this study is syncing the pianist performance with digital music notes. I picked MIDI as the standard. This section reviews the process of extracting MIDI messages. Many open-source libraries offer MIDI file parsing functions, which use the same way for reading files, but their methods for showing MIDI events could be different. I selected PrettyMIDI ${ }^{1}$ for this study that extracts MIDI instruments and their notes separately. Each MIDI event contains the following attributes:

- Velocity (type:integer): the sound volume for playing this specific event.

- Pitch (type:integer): the MIDI note number (see table 2.1).

- Note-on/off (type:float): the start and end time of playing each event.

For each piece, I collected the time series of digital notes (TN) by looping through MIDI messages and picked the note-on values. For chords ${ }^{2}$, all music notes should be played simultaneously, since all pitches has the same note-on values, I kept the first pitch and discarded the rest. Sometimes, a short time gap would happen between pressing chord keys, which the timeshifting mechanism that I will explain later resolves this time latency.

\subsection{Time Series of Pressed Keys (TP)}

I have already proposed two methods for finding signal peaks in section 4.3.3. The time series of pressed-keys (TP) is nothing more than the aggregation of

\footnotetext{
${ }^{1}$ http://github.com/craffel/pretty-midi

${ }^{2} \mathrm{~A}$ group of three or more notes played simultaneously.
} 


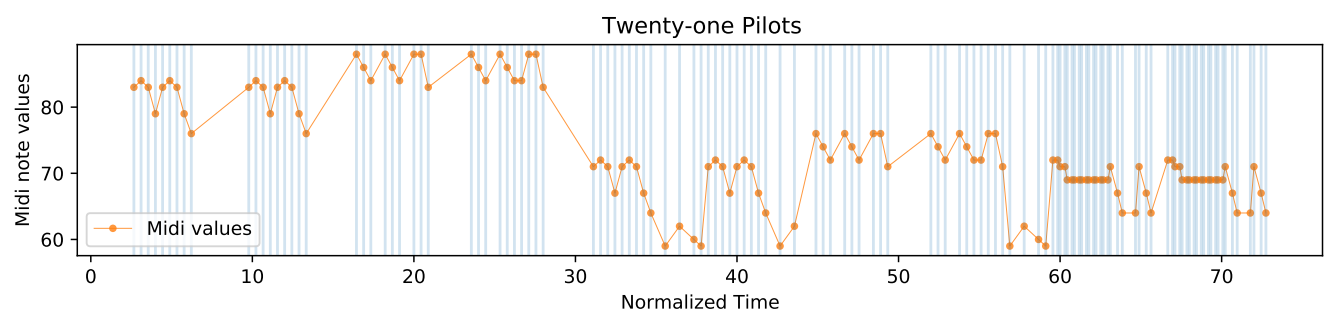

Figure 5.2: Time series of digital notes for Twenty-one Pilots piece with the MIDI values.

the founded peaks. In experimental sessions, I utilized the K-means clustering and some other semi-heuristics methods. The following processes show the data preparation before applying clustering method (see fig. 5.4).

- For reducing the accelerometer random noise, I used the simple moving average filter (see section 3.2.2).

- As we discussed before, by putting the accelerometer on the flat surface, the value of z-axis is $\approx 9.81$. However, based on the IMU device's position and orientation on wrists, the acceleration signal's mean value could be different. So, I calculated the mean values of the whole signal and then subtracted all points by that. This method shifts the signal mean close to 0 .

- As mentioned earlier in section 3.2.2, the accelerometer sensor records both acceleration and deacceleration values. My observation showed that the acceleration resulting from pressing the piano keys generates signal peaks with higher values (in positive range) than deacceleration. Therefore, keeping the negative values is unnecessary and increased the calculation time. I discarded all the negative values of the acceleration 
signal and replaced them with 0 .
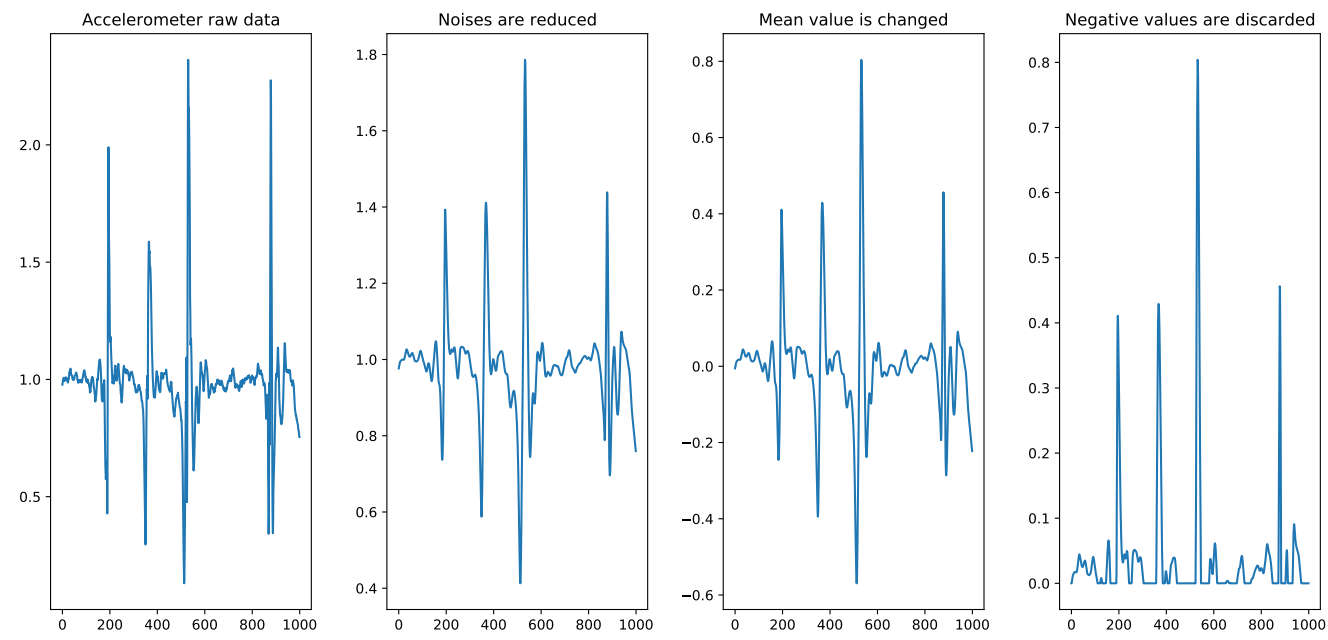

Figure 5.3: Data preparation for peak detection process.

In general, signal processing filters such as simple moving average that I used for noise reduction only change the value of data points, and the number data points remains fixed. In the data collection phase, I mentioned that the frequency of recording is $200 \mathrm{hz}$. So, system saves 200 points every second. Since only the peaks are needed, I used another heuristic method to increase the calculation speed and execution performance by removing unnecessary data points. The heuristic method checked all data points one by one and compared each value with the previous and next data points. If values were between the previous and next data point, so keeping that data point was unnecessary. In other words, this method detected the minimum and maximum nodes of each spike and discarded all values between these two points. 

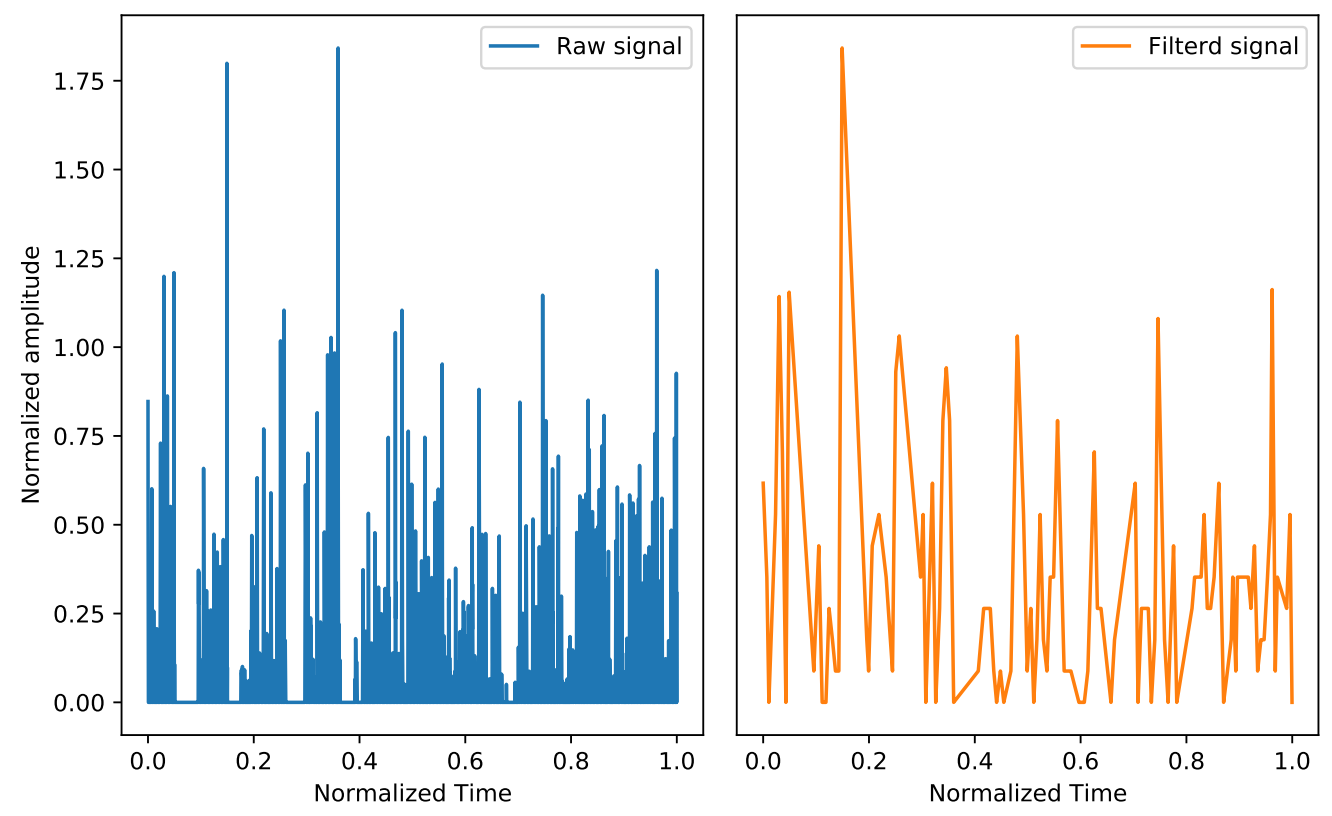

Figure 5.4: A Heuristic filter is applied to the raw accelerometer signal to keep the peaks and reduce the unnecessary data points. 


\subsection{Time Lag}

In a solo piano performance, the start of playing a piece depends entirely on the musician's decision. Even though we defined a unique gesture (see section 4.2) as indicators, the time delay caused by rushing or dragging at the beginning of the play can remain up to the end of the performance. Also, in case of having rests at the beginning of the piece for any hands, the left and right hands may not start to play at the same time.

The wrist-twisting gesture solved the latency problem of recording data; however, the problem of syncing each hand with the first notes still exists. To solve this problem, we must first understand pianists' possible behaviours after wrist-twisting and before starting to play. I identified three possible actions at this stage.

- Pianists could immediately start to play after twisting their wrists.

- Pianists could start to play with a short delay after twisting their wrists.

- Pianists could twist their wrists and move their hand over keys to the start point and then start to play.

The only possible way to detect the start time in all mentioned scenarios is to find the first positive spike in the acceleration signal, which the first pressed key generated that. During the gesture detection step, I discarded all data before the first and after the last detected points. To find the first pressed-key we can apply the similar method but this time to the acceleration data. In fig. 5.5, the gyroscope and accelerometer data are shown on the left and the right sides, respectively. Since the recording frequencies of both were 
the same, we can use the WT-gesture guideline for other series. The first pointed value in the right plot indicated the first pressed key after the WT had occurred.
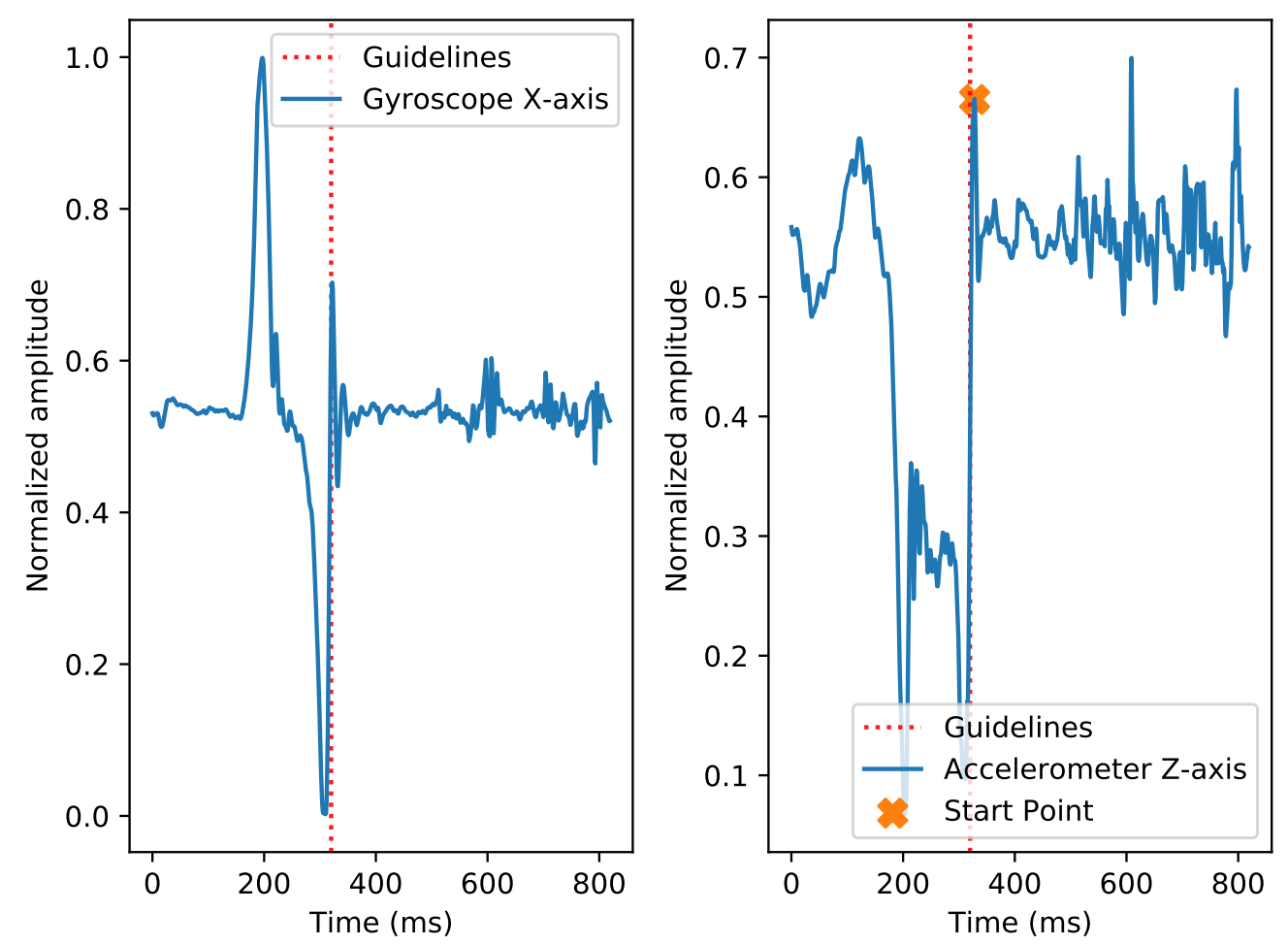

Figure 5.5: Shows the gyroscope (left) and the accelerometer (right) data of the same record. The WT-gesture detection guideline used for accelerometer to find the first pressed key.

Finding the first pressed-key and aligning that with the first note solved the dragging and rushing problem. However, my experiments showed that not only dragging and rushing problems exist at the beginning of the play but also occur during the performance. In the next section, we will explore different approaches to find time-lags during the play and solutions to reduce them. 


\section{$5.4 \quad$ Time Shifting}

Humans are not capable of controlling time as accurately as computers. Suppose all pressed-keys generated significant spikes on the acceleration signal, and the system detected all peaks. Nonetheless, the time latency resulting from dragging or rushing while playing are inevitable and affect syncing progress. The algorithm for resolving the dragging and rushing problem was improved throughout this study, which ultimately resulted in higher accuracy and lower error rates. I categorized the whole process into three attempts.

\subsubsection{Attempt 1}

After collecting hand movements in experimental sessions and detecting the first and last wrist-twisting gestures, I searched for the first signal peak in acceleration signal. I assumed that the first pressed-key generated this peak, so I marked that as the start point. In the first time-shifting approach, I set the value of start point to 0 , and then subtracted all other data points in time series of pressed-keys (TP) by the original value of start point. This approach synchronizes the start point of both TP and time series of digital notes (TN). Then, for making both TP and TN series comparable, I normalized them in a range of $[0-1]$.

Next, I applied the dynamic time warping (DTW) function to TN and TP series. DTW function returned the optimal alignment path in a shape of list of pairs. Each pair is pointed to the specific indexes in TN and TP, respectively. I used root mean square error for calculating the average alignment error for this approach (see fig. 5.8). 

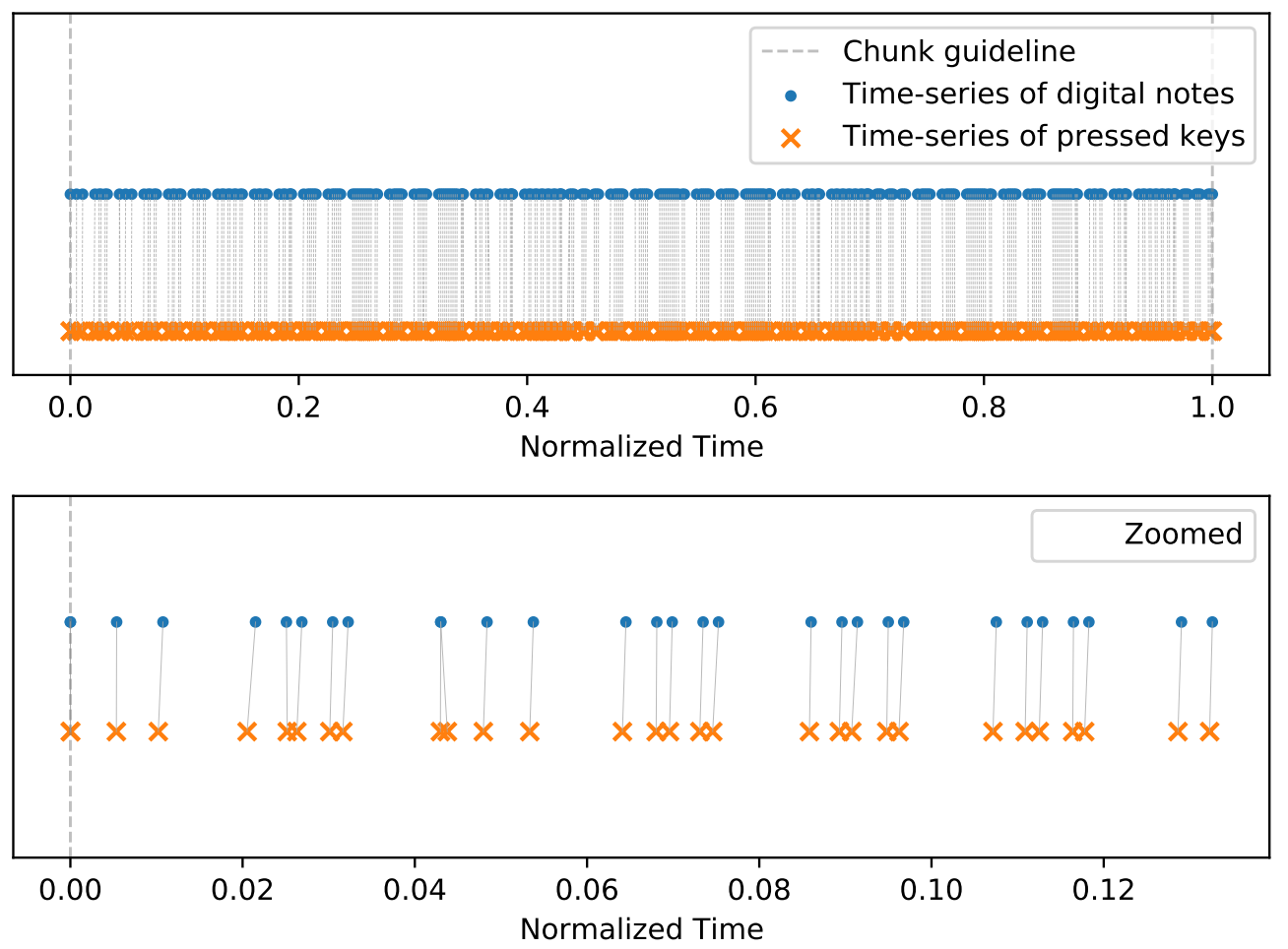

Figure 5.6: The top plot shows the result of applying DTW to TN and TP series. The bottom plot is the zoomed of the top one.

\subsubsection{Attempt 2}

The previous step only synced the first note, and the result indicated the average error for the whole piece. The error was not sufficient to make a decision for page-turning. In the second approach, I split all data into equal chunks. I applied cross-correlation to each chunk to find better alignment of TN and TP. This phase increased the possibility of series data point alignment and reduced the error rate. 

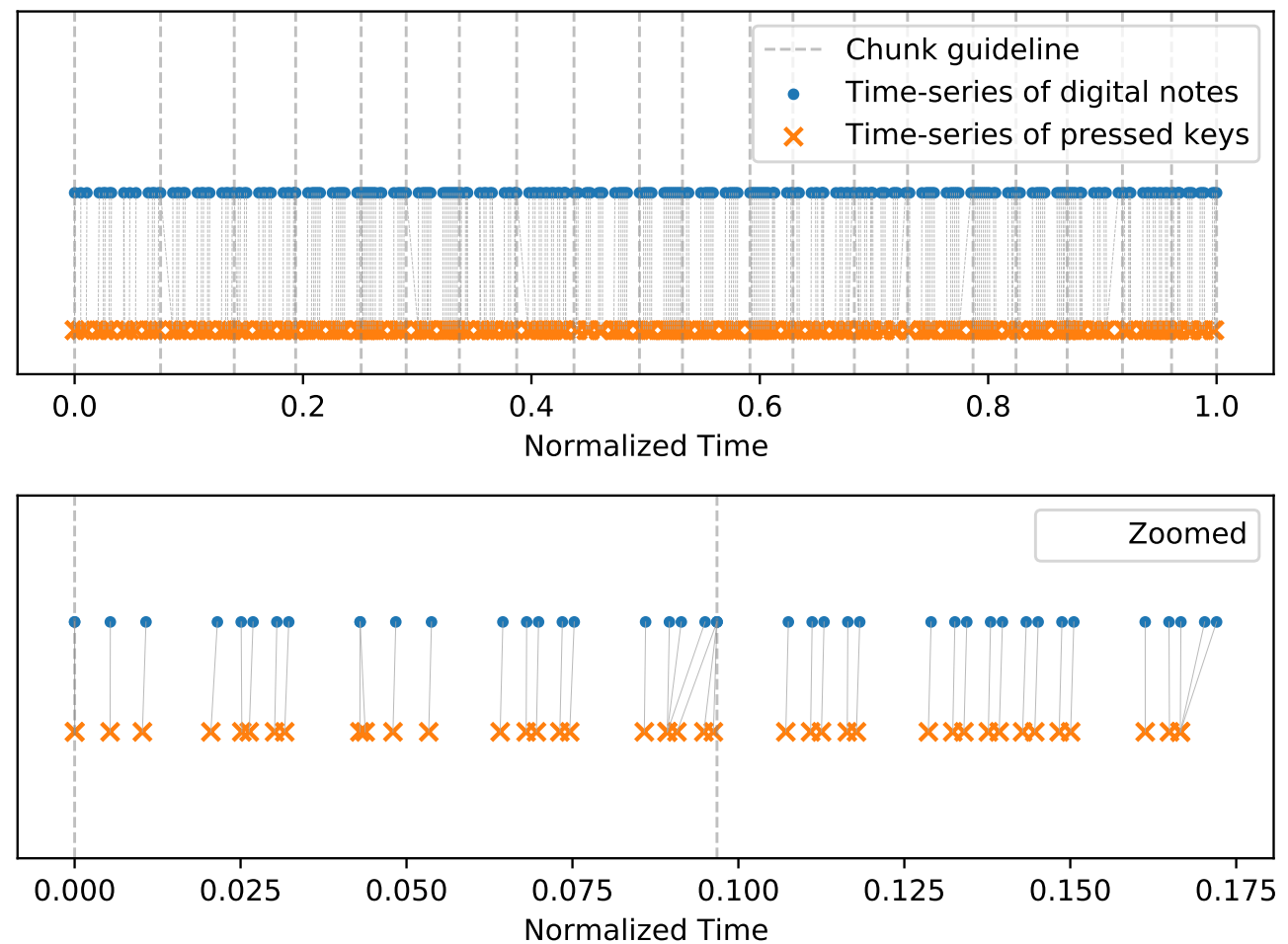

Figure 5.7: The top plot shows the DTW function applied to the equal chunks of TN and TP series. The bottom plot is the zoomed of the top one.

\subsubsection{Attempt 3}

The main issue of the second step was selecting the right number for chunking data. If any chunk contains long notes or rests, the small number of misalignment generates significant errors. I suggested the auto-chunking method, which tries to find the optimum cut and chunks the time series of digital notes based on notes delta time. So the notes with similar delta time are clustered in the same group. Since time is a relative concept, my suggested method can cope with different types of music.

The auto-chunking process starts by measuring time differences (TD) between all notes. K-means function with $k=2$ clusters TD array into 
short and long clusters, which data in long cluster define the boundaries of auto-chunking. For increasing the accuracy, I applied the cross-correlation function for all chunks like the previous phase.
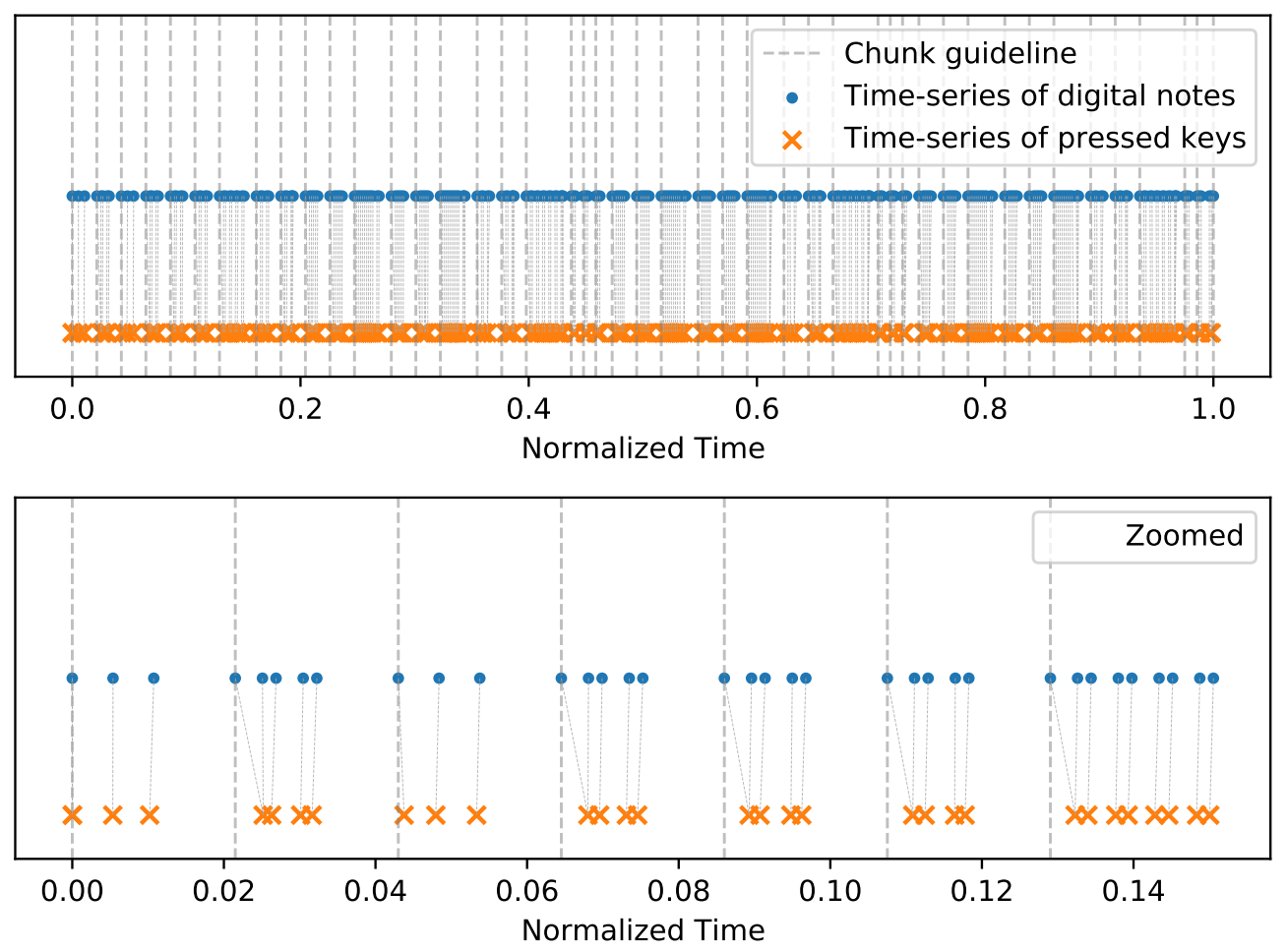

Figure 5.8: The top plot shows the result of applying the DTW function to the dynamic chunks of TN and TP collection. The bottom plot is the zoomed of the top one. 


\subsection{Online Mode}

In all previous sections, we discovered details of tuning the automatic pageturning. This section will first discuss the differences between that stage (offline mode) and online mode, and then I will suggest some solutions for utilizing the existing code in a real-time context. Fig 5.9 shows the workflow of my proposed solution for the online automatic page-turner.

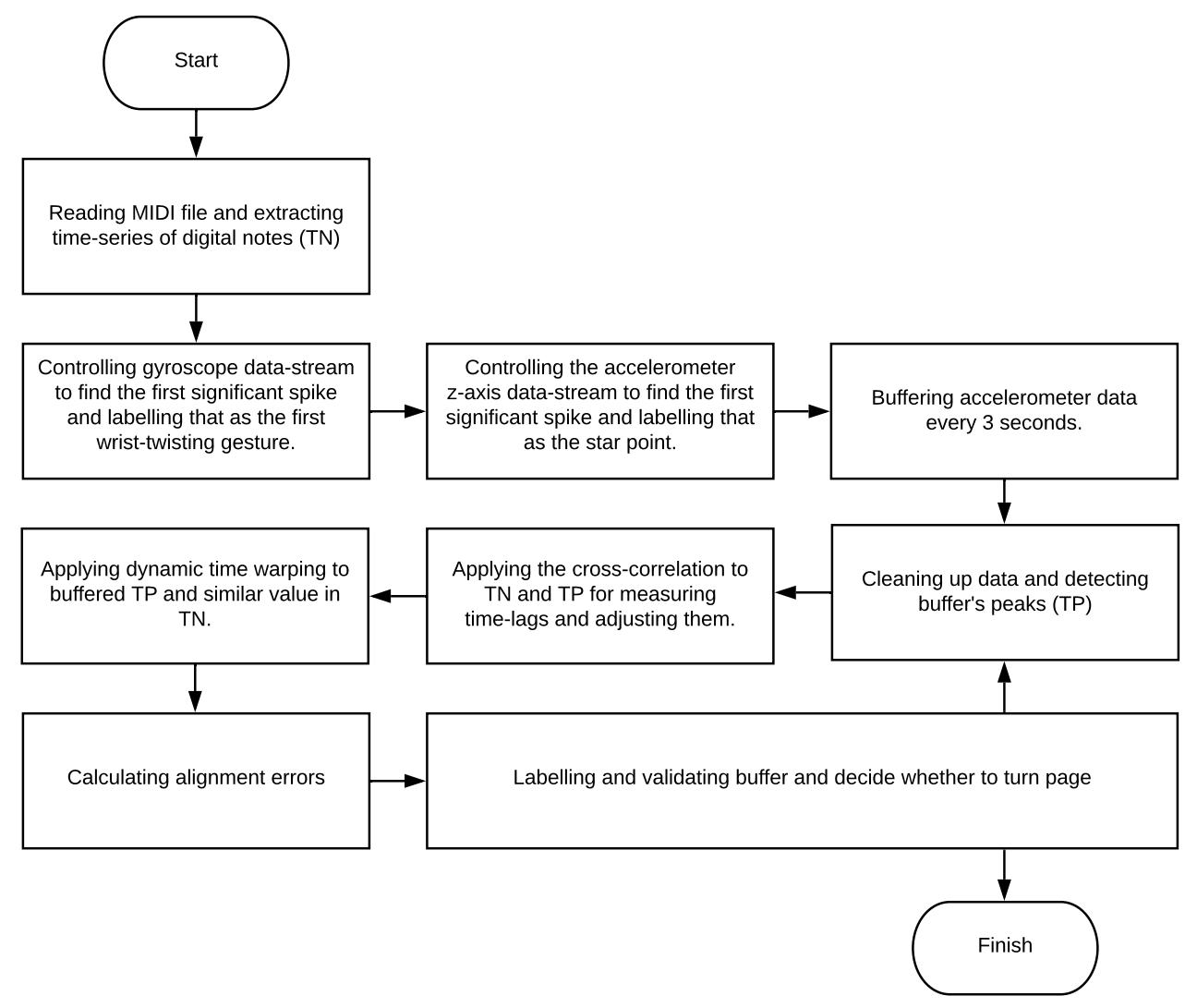

Figure 5.9: Workflow of automatic page-turning in online mode. 


\subsubsection{Data Stream}

Unlike the previous stage, where all data was available before the analysis stage, in the online mode, data is constantly added to the system, which creates some limitations:

- Normalization: in offline mode, for making TN and TP comparable, I normalized all data. Since there is no information about future data of $\mathrm{TP}$, data normalization is not practical in online form.

- Gesture detection: WT gestures in offline mode determined the boundaries of valid data for analysis. Detecting the last gesture is not mandatory in online mode (because there is no page to turn at the end of the performance), so finding the first WT gesture would be enough to start the syncing process.

- Chunking: auto-chunking method is also unavailable in online mode. I will discuss the strategy of clustering notes in the next section.

- Peak detection: the mentioned approaches for identifying peaks in offline mode (see section 4.3.3) returns invalid results in online mode.

In this section, I used the buffering mechanism to overcome the data loading limitation. In the computer context, the buffer refers to the fixed amount of memory for storing data temporarily before starting a process. The recording frequency of IMU sensors during experimental sessions was set to $200 H z$, which means both accelerometers and gyroscopes transmitted 200 data every second. For online mode, I set the buffer size (chunk) to 600, so system processes the received data every 3 second. (see fig. 5.10). 

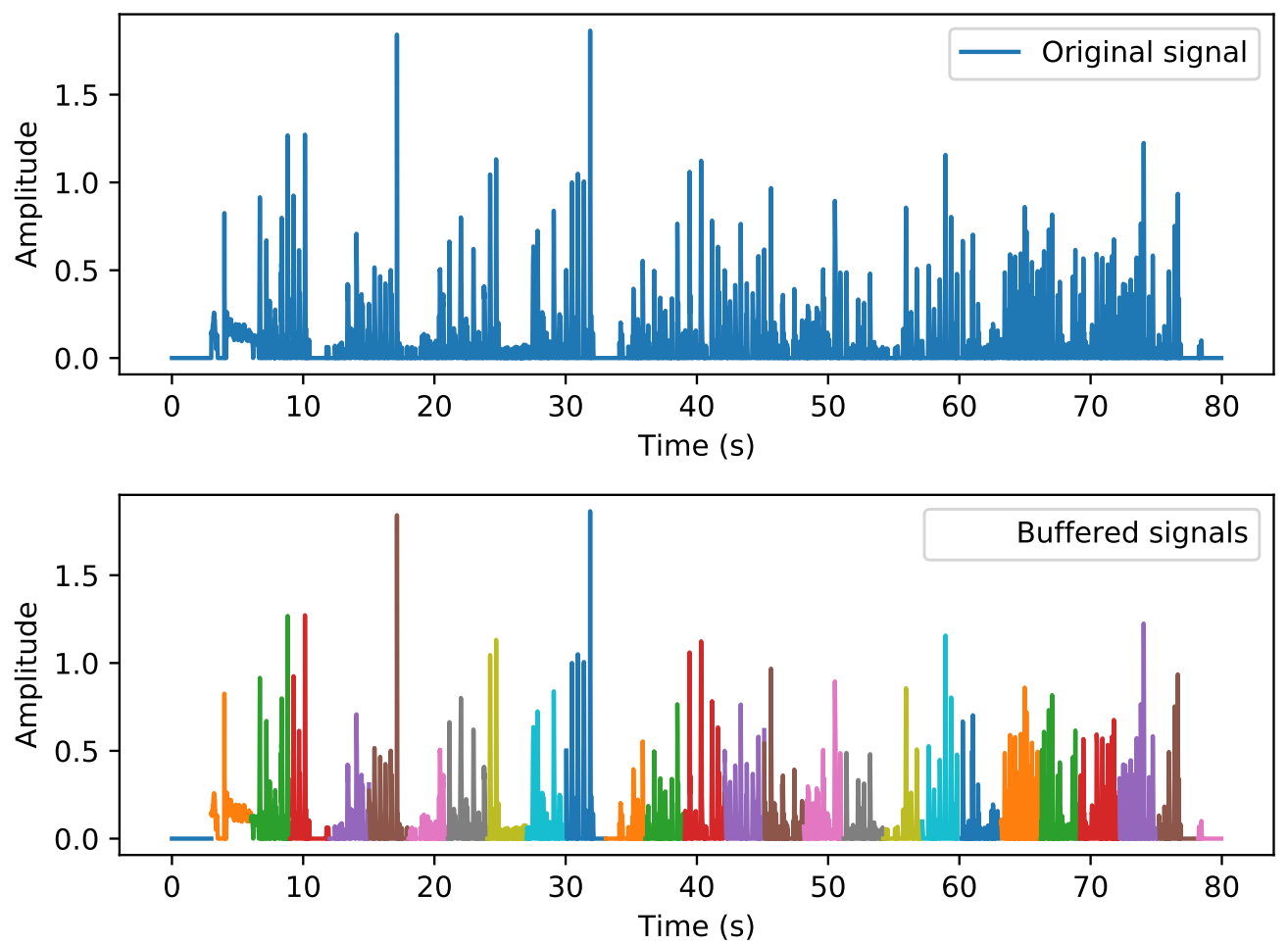

Figure 5.10: Original signal (top) and the same signal in the buffered mode (down).

\section{Peak Detection}

Like offline mode, I utilized the moving average filter for reducing noises of accelerometer data on each buffer with a fixed window. The geometric angle and k-means clustering solutions for detecting signal peaks returned invalid results, so I added another heuristic methods for removing unnecessary data and finding signal spikes (see section 5.2). First, I found all local maximum values by comparing each value with its neighbours $\left(x_{1}=\max\right.$ if $x_{0}<x_{1}>$ $0 x_{2}$ ). This process identified many peaks; however, the valid spikes had the highest values. I traced all detected local maximums trends to find the peaks with the highest values, and I labelled them as valid peaks (see fig. 5.11). 
(a)

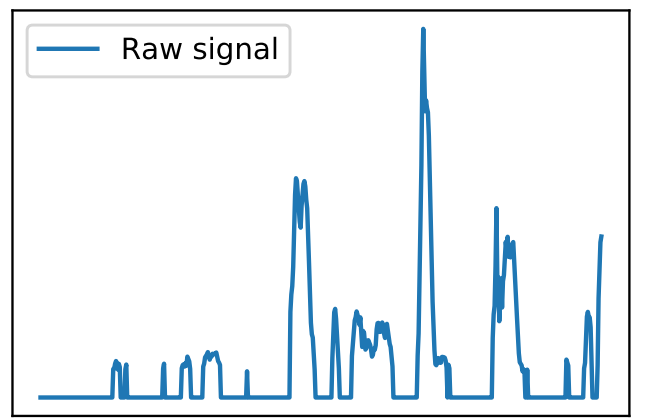

(c)

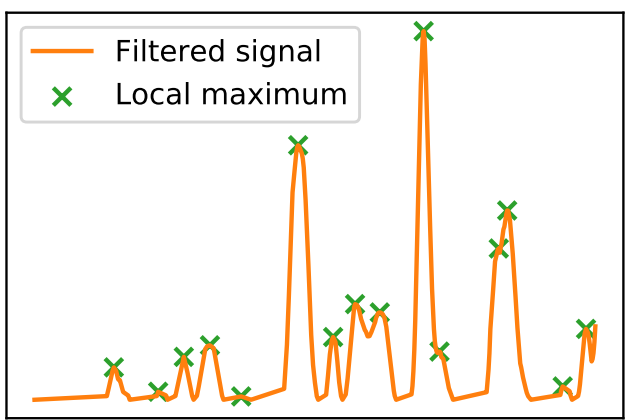

(b)

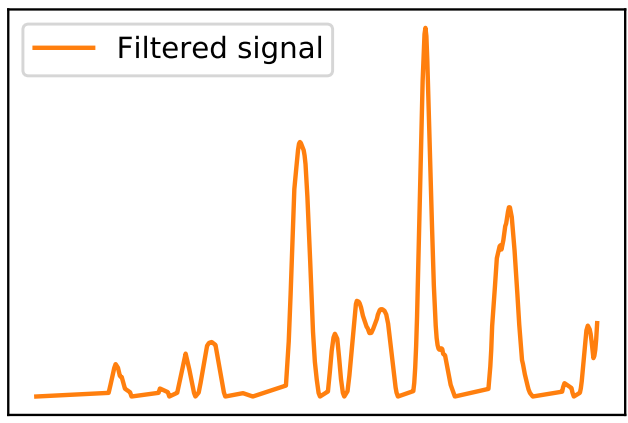

(d)

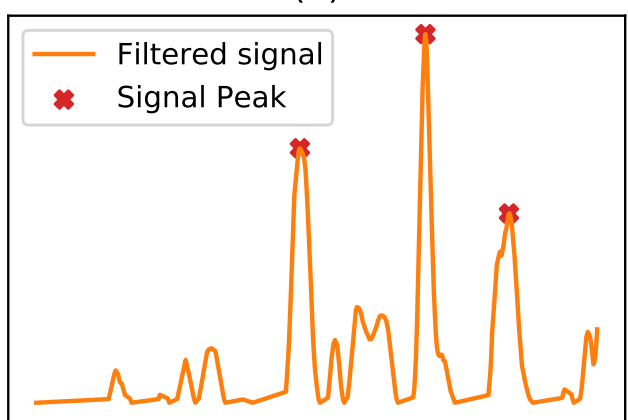

Figure 5.11: Four steps of filtering the accelerometer signal to detect signal peaks in online mode.

\section{Data alignment and decision model}

The auto-chunking is not available in online mode. When each buffer is filled, I assumed that the piece is ended, and I handled that like the offline mode. Therefore, I reused most of the offline mode function. In next chapter, I will visualize the results of experimental session. 


\section{Chapter 6}

\section{Results}

I described all parts of the suggested solution for an automatic page-turner in detail in previous chapters. Like other academic research, I tried to utilize different methods from related studies. I observed some gaps in methods in some cases, so I developed some software for those specific points. The initial results were invalid, and the error rates were significantly high. Therefore, as a researcher, I tried to improve codes continuously. This chapter reviews the results of all processes of designing the automatic page-turner individually. 


\subsection{WT-gesture}

As I described earlier in section 4.2, I defined a unique wrist gesture, which participants must perform at the beginning and the end of each play. This method determines the boundaries of valid data on each run. Since the length of music pieces was different, I normalized all data in a range of $[0-100]$. The golden dreams and sonatina Op. 20 got the minimum and maximum mean absolute deviations. Fig 6.1 shows that average accuracy is $99.99 \%$ which means that WT gesture detected the right boundaries of data and pianists played all pieces with the right and accurate tempo.

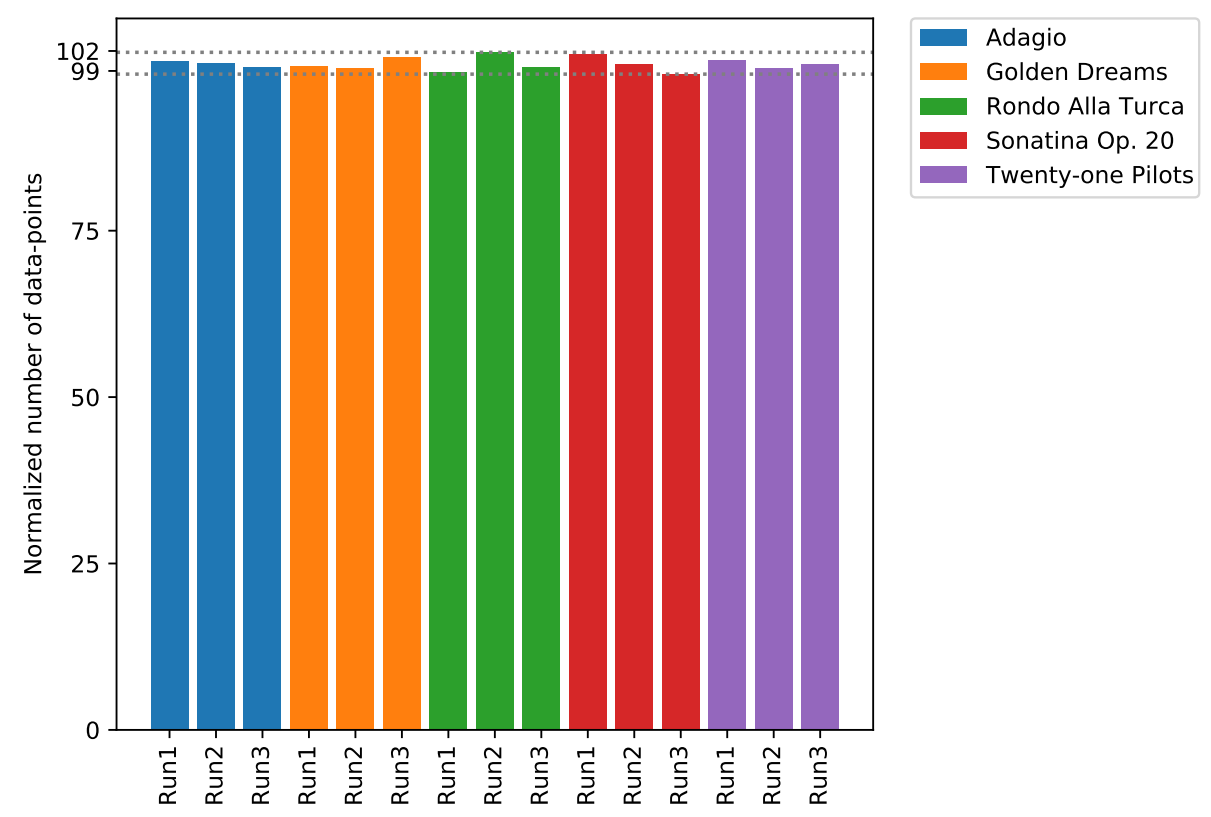

Figure 6.1: Shows the normalized number of acceleration data after applying WT-gesture function for all played pieces. 


\subsection{Peak Detection}

Clustering peaks of accelerometer signal is the next process. I suggested Kmeans and geometric angle analysis in section 4.3.3. For K-means method, the percentage of detected peaks to actual notes numbers is shown in fig. 6.2. Results revealed that the system found more peaks in the first run of Sonatina Op.20 to actual notes. Also, K-means approach could not detect around $20 \%$ of peaks for all runs of Adagio.

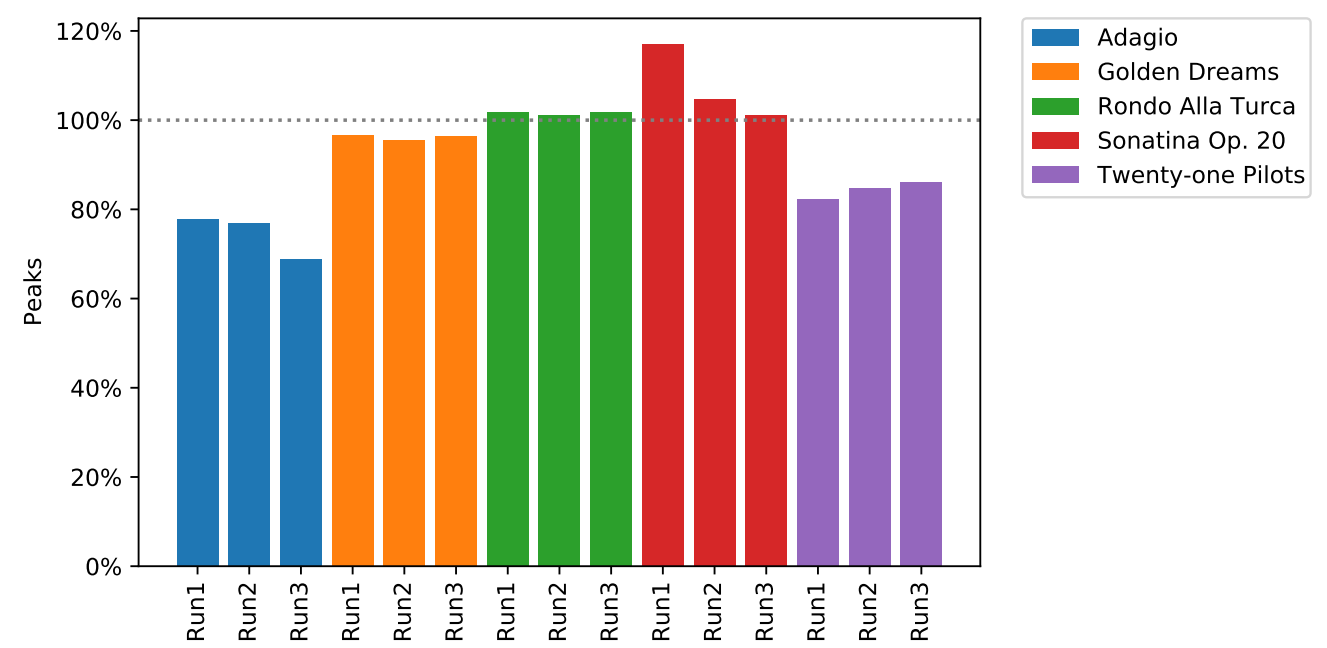

Figure 6.2: Shows the percentage of detected peaks to actual music notes with K-means method.

In another approach, I measured the geometry angles of data points to find signal peaks. I categorized range of angles into $\left[0-5^{\circ}\right],\left[5-10^{\circ}\right],\left[10-15^{\circ}\right]$, $\left[15-20^{\circ}\right]$, and $\left[20^{\circ}+\right]$ (see fig. 6.5a). The wide range of angles extracted by this method explained that the geometric angle detection is not an accurate solution for identifying signal peaks in the context of my study, so I rejected this approach. 


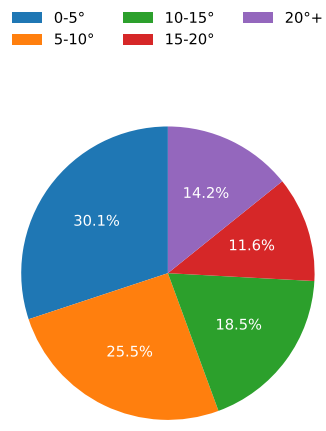

Adagio

$$
\begin{array}{r}
0-5^{\circ} \\
5-10^{\circ} \\
15-25^{\circ} \\
15-20^{\circ}
\end{array}
$$

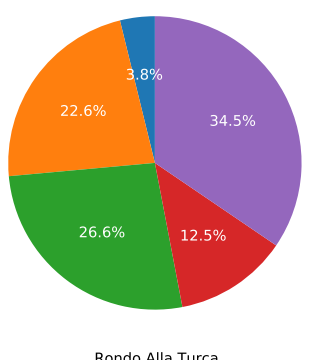

Rondo Alla Turca

$=\begin{aligned} & 0-5^{\circ} \\ & 5-10^{\circ} \\ & 15-15^{\circ} \\ & 15-20^{\circ}\end{aligned}$

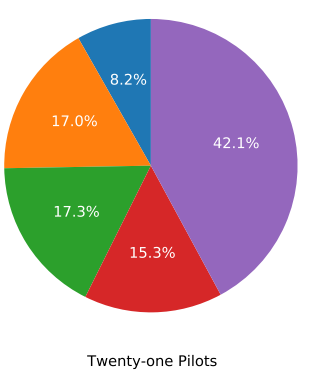

$\begin{gathered}0-5^{\circ} \\ 5-10^{\circ} \\ 15-20^{\circ}\end{gathered} 0^{\circ}=$

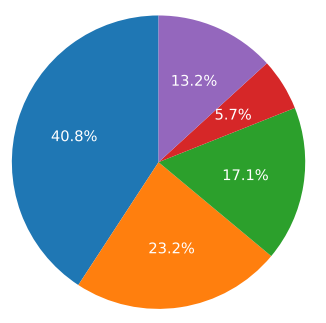

Golden Dreams

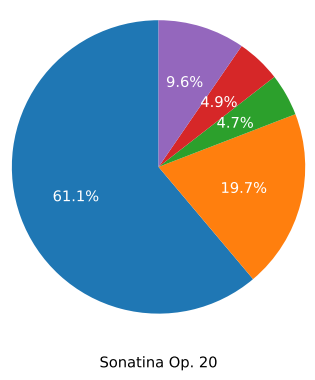

Sonatina Op. 20

(a) The percentage of detected peaks with geometric angle calculation. 


\subsection{Time Shifting}

I explained the source of time-lags in section 5.4, and also I proposed three attempts (no chunking, equal chunking, and auto-chunking) to measure and reduce time lags. In this part, I implemented all attempts and categorized them based on the DTW distance and alignment errors (see section 6.3).

\begin{tabular}{lc|cc|cc|ccc}
\hline & Run & \multicolumn{2}{|c|}{ Attempt \#1 } & \multicolumn{2}{c}{ Attempt \#2 } & \multicolumn{2}{c}{ Attempt \#3 } \\
Music Piece & \# & dd & err\% & dd & err\% & dd & err\% \\
\hline Adagio & 1 & 113 & 0.89 & 40 & 2.50 & 113 & 0.00 \\
Adagio & 2 & 115 & 2.61 & 39 & 0.00 & 115 & 0.00 \\
Adagio & 3 & 117 & 4.27 & 43 & 6.98 & 119 & 0.00 \\
Golden Dreams & 1 & 297 & 0.67 & 39 & 0.00 & 299 & 0.00 \\
Golden Dreams & 2 & 306 & 3.59 & 51 & 12.5 & 317 & 0.00 \\
Golden Dreams & 3 & 300 & 1.67 & 39 & 0.00 & 304 & 0.00 \\
Rondo Alla Turca & 1 & 185 & 8.11 & 45 & 0.01 & 188 & 0.00 \\
Rondo Alla Turca & 2 & 181 & 6.08 & 46 & 0.00 & 184 & 0.00 \\
Rondo Alla Turca & 3 & 178 & 4.49 & 41 & 4.88 & 181 & 0.00 \\
Sonatina Op. 20 & 1 & 367 & 24.52 & 44 & 0.01 & 374 & 0.00 \\
Sonatina Op. 20 & 2 & 329 & 15.81 & 40 & 0.00 & 332 & 0.00 \\
Sonatina Op. 20 & 3 & 320 & 13.44 & 40 & 0.00 & 324 & 0.00 \\
Twenty-one Pilots & 1 & 130 & 0.00 & 40 & 2.50 & 133 & 0.63 \\
Twenty-one Pilots & 2 & 135 & 3.70 & 48 & 0.00 & 136 & 0.21 \\
Twenty-one Pilots & 3 & 132 & 1.52 & 40 & 0.00 & 132 & 0.00 \\
\hline
\end{tabular}

Table 6.1: DTW distances (dd) and alignment errors (err) for all runs with different time-shifting approaches.

The results show that the average error rates decreased with each new attempt. Only the Adagio, in the second attempt, the error increased slightly. However, in the third stage, the error reached zero. 
Table 6.2 shows the percentage of each DTW alignment types for all runs with different time-shifting approaches.

\begin{tabular}{|c|c|c|c|c|c|c|c|c|c|c|}
\hline \multirow[t]{3}{*}{ Music Piece } & \multirow{3}{*}{$\begin{array}{c}\text { Run } \\
\#\end{array}$} & \multicolumn{3}{|c|}{ Attempt 1} & \multicolumn{3}{|c|}{ Attempt 2} & \multicolumn{3}{|c|}{ Attempt 3} \\
\hline & & $\mapsto$ & $\leftrightarrow$ & $\rightarrow$ & $\mapsto$ & $\leftrightarrow$ & $\rightarrow$ & $\mapsto$ & $\leftrightarrow$ & $\rightarrow$ \\
\hline & & $\%$ & $\%$ & $\%$ & $\%$ & $\%$ & $\%$ & $\%$ & $\%$ & $\%$ \\
\hline Adagio & 1 & 70.8 & 5.31 & 23.89 & 93.16 & 1.71 & 5.13 & 38.05 & 23.58 & 38.37 \\
\hline Adagio & 2 & 65.22 & 8.7 & 26.09 & 94.74 & 4.39 & 0.88 & 40.96 & 21.3 & 37.74 \\
\hline Adagio & 3 & 63.25 & 1.71 & 35.04 & 90.4 & 1.6 & 8 & 39.07 & 20.63 & 40.3 \\
\hline Golden Dreams & 1 & 83.5 & 12.12 & 4.38 & 89.9 & 9.43 & 0.67 & 55.79 & 25.46 & 18.75 \\
\hline Golden Dreams & 2 & 90.2 & 1.63 & 8.17 & 86.29 & 8.41 & 5.3 & 66.48 & 13.21 & 20.31 \\
\hline Golden Dreams & 3 & 88 & 6.33 & 5.67 & 89.51 & 8.2 & 2.3 & 67.41 & 13.38 & 19.21 \\
\hline Rondo Alla Turca & 1 & 90.27 & 2.7 & 7.03 & 92.27 & 2.06 & 5.67 & 79.59 & 5.99 & 14.42 \\
\hline Rondo Alla Turca & 2 & 92.27 & 2.21 & 5.52 & 90.05 & 3.66 & 6.28 & 80.61 & 4.78 & 14.61 \\
\hline Rondo Alla Turca & 3 & 91.57 & 5.06 & 3.37 & 95.48 & 3.95 & 0.56 & 74.22 & 10.8 & 14.98 \\
\hline Sonatina Op. 20 & 1 & 84.2 & 3.81 & 11.99 & 93.5 & 5.42 & 1.08 & 29.81 & 26.08 & 44.11 \\
\hline Sonatina Op. 20 & 2 & 85.41 & 2.43 & 12.16 & 90.7 & 4.36 & 4.94 & 22.42 & 28.23 & 49.35 \\
\hline Sonatina Op. 20 & 3 & 82.81 & 4.38 & 12.81 & 91.18 & 2.35 & 6.47 & 8.92 & 36.97 & 54.12 \\
\hline Twenty-one Pilots & 1 & 80 & 1.54 & 18.46 & 93.94 & 3.03 & 3.03 & 86.82 & 3.68 & 9.5 \\
\hline Twenty-one Pilots & 2 & 78.52 & 2.22 & 19.26 & 96.32 & 1.47 & 2.21 & 91.3 & 0.42 & 8.28 \\
\hline Twenty-one Pilots & 3 & 80.3 & 3.79 & 15.91 & 97.71 & 1.53 & 0.76 & 86.91 & 4.21 & 8.88 \\
\hline
\end{tabular}

Table 6.2: Shows different DTW alignment types for all runs with different time-shifting approaches. $(\mapsto)$ symbol represents the one-to-one alignment, $(\leftrightarrow)$ symbol shows the fully aligned connection, and $(\rightarrow)$ symbol indicates the one-to-many connections. 


\subsection{Decision Model}

I set the page-turning event in the middle of each piece. Table 6.3 shows all necessary data for the page-turning decision model. Based on decision model criteria (see section 4.4.4), all nominated chunks for page-turning got the passed label. Next, the sum of one-to-one and aligned connections was also greater than the minimum range(50\%). So, all nominated chunks satisfied the minimum requirements, and the page-turning process has entirely done for each piece. I also visualized alignments of all pieces in figs. 6.6 to 6.10 .

\begin{tabular}{lc|cc|ccc|c}
\hline Music Piece & $\begin{array}{c}\text { Run } \\
\text { \# }\end{array}$ & $\begin{array}{c}\text { Total } \\
\text { chunks }\end{array}$ & $\begin{array}{c}\text { Middle } \\
\text { chunk }\end{array}$ & $\begin{array}{l}\boldsymbol{\text { \% }} \\
\text { \% }\end{array}$ & $\begin{array}{c}\text { \% } \\
\text { \% }\end{array}$ & $\begin{array}{c}\text { Valid } \\
>50 \%\end{array}$ \\
\hline Adagio & 1 & 28 & 14 & 100 & 0 & 100 & $\checkmark$ \\
Adagio & 2 & 28 & 14 & 100 & 0 & 100 & $\checkmark$ \\
Adagio & 3 & 28 & 14 & 100 & 0 & 100 & $\checkmark$ \\
Golden Dreams & 1 & 44 & 22 & 66 & 16 & 82 & $\checkmark$ \\
Golden Dreams & 2 & 44 & 22 & 71 & 0 & 71 & $\checkmark$ \\
Golden Dreams & 3 & 44 & 22 & 66 & 16 & 82 & $\checkmark$ \\
Rondo Alla Turca & 1 & 18 & 9 & 83 & 0 & 82 & $\checkmark$ \\
Rondo Alla Turca & 2 & 18 & 9 & 80 & 0 & 80 & $\checkmark$ \\
Rondo Alla Turca & 3 & 18 & 9 & 50 & 16 & 66 & $\checkmark$ \\
Sonatina Op. 20 & 1 & 76 & 38 & 60 & 20 & 80 & $\checkmark$ \\
Sonatina Op. 20 & 2 & 76 & 38 & 66 & 0 & 66 & $\checkmark$ \\
Sonatina Op. 20 & 3 & 76 & 38 & 66 & 0 & 66 & $\checkmark$ \\
Twenty-one Pilots & 1 & 16 & 8 & 0 & 100 & 100 & $\checkmark$ \\
Twenty-one Pilots & 2 & 16 & 8 & 0 & 100 & 100 & $\checkmark$ \\
Twenty-one Pilots & 3 & 16 & 8 & 0 & 100 & 100 & $\checkmark$ \\
\hline
\end{tabular}

Table 6.3: The necessary data for decision making for turning page at the middle of each piece (offline mode). The $\mapsto, \leftrightarrow$ symbols represent one-toone and aligned connections respectively. 


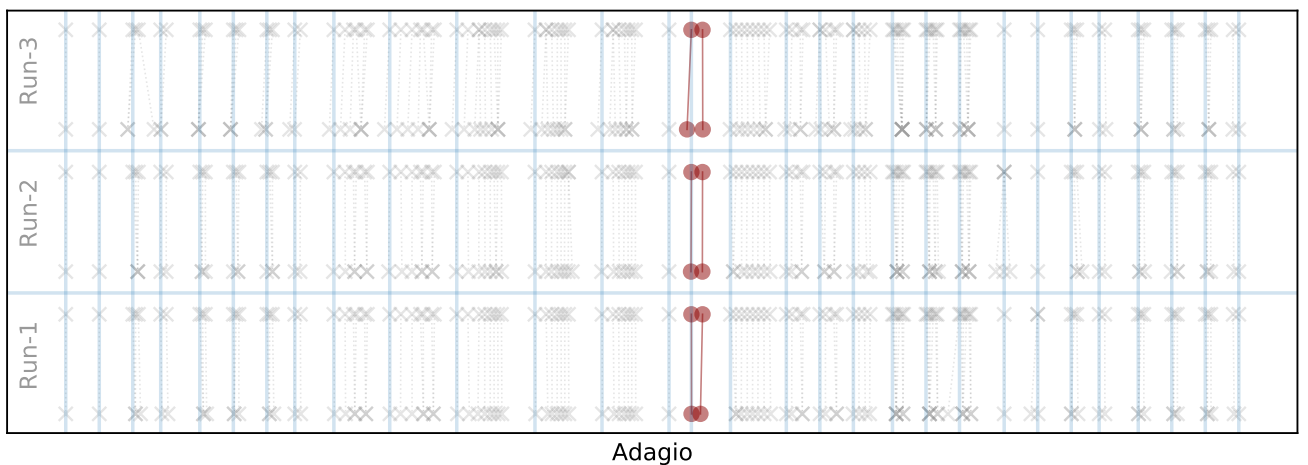

Figure 6.6: The final alignment of Adagio for all runs. Red shapes indicates the turning-page points.

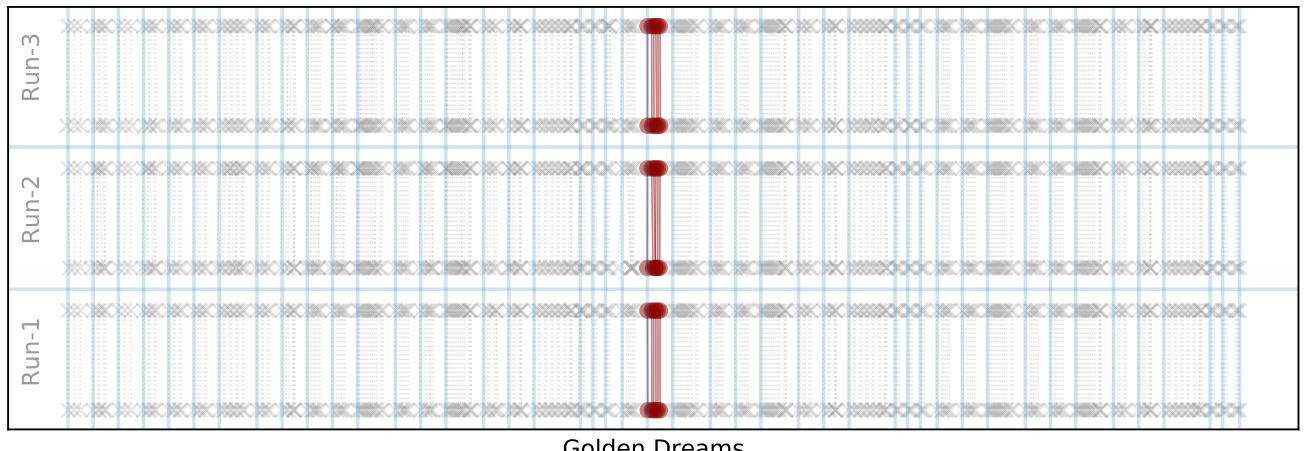

Golden Dreams

Figure 6.7: The final alignment of Golden Dreams for all runs. Red shapes indicates the turning-page points.

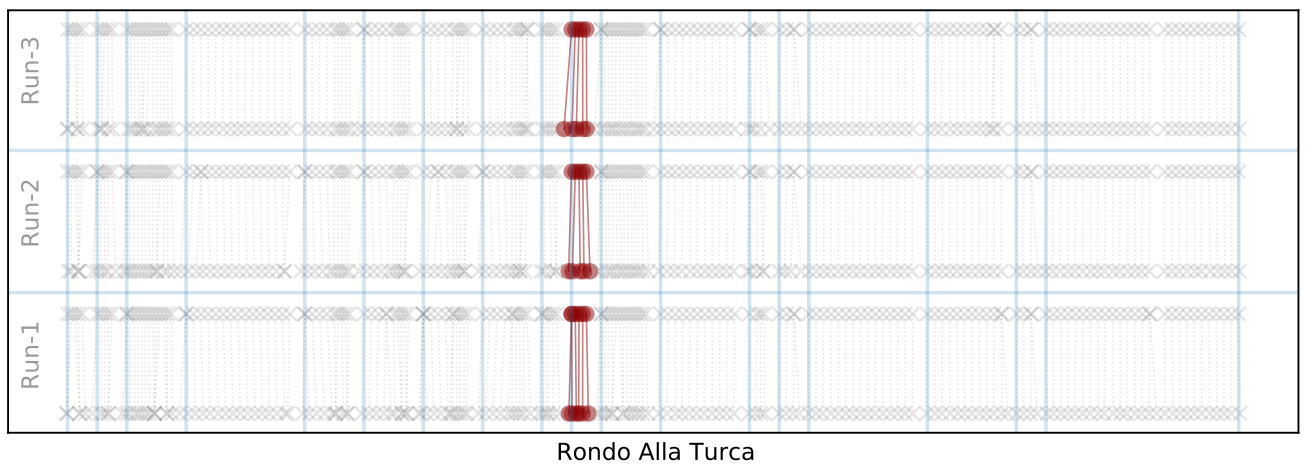

Figure 6.8: The final alignment of Rondo Alla Turca for all runs. Red shapes indicates the turning-page points. 


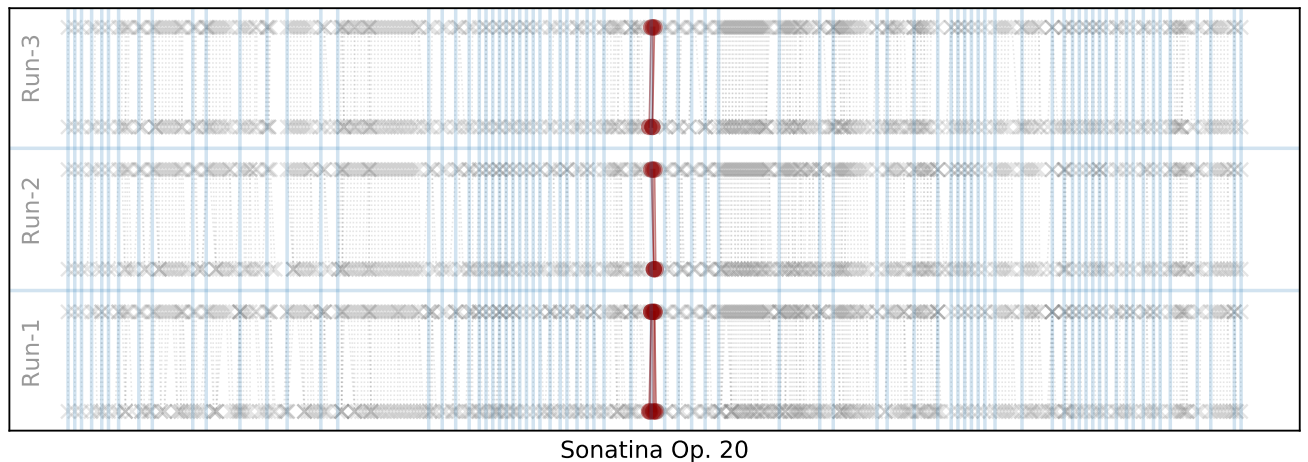

Figure 6.9: The final alignment of Sonatina $O p$. 20 for all runs. Red shapes indicates the turning-page points.

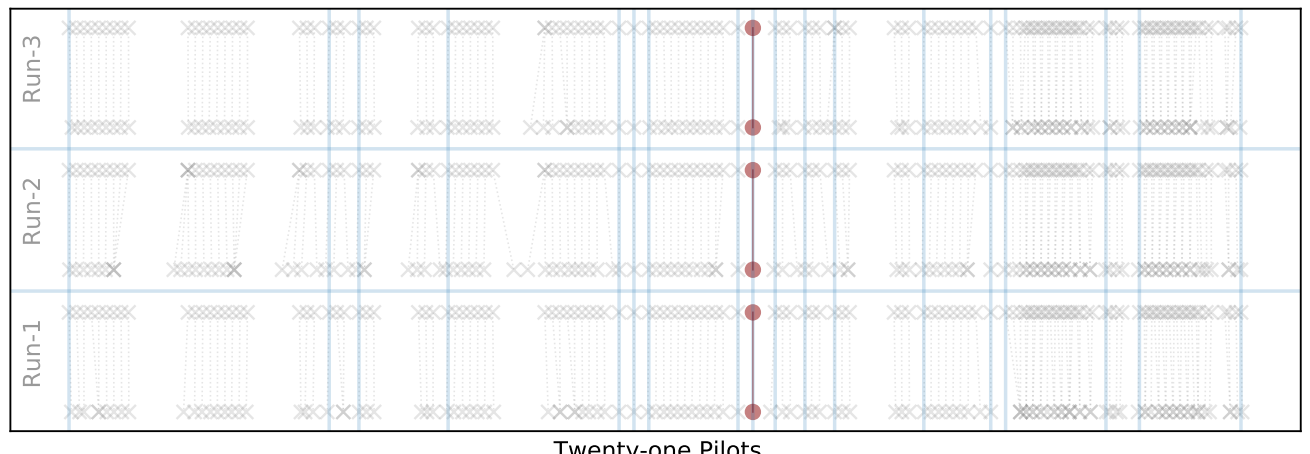

Figure 6.10: The final alignment of Twenty-one Pilots for all runs. Red shapes indicates the turning-page points. 


\subsection{Online Mode}

I have explained details of online mode in section 5.5 I set the buffer size to 600, so the decision model checked data every 3 seconds. Like offline mode, I set one page-turning event in the middle of each piece. Table 6.4 shows the details of aligned pairs at that point. The online mode algorithm starts to process after detecting the first wrist-gesture and the first key pressed. I performed all the music pieces in different styles, so the buffer number identified as the middle buffer is different for each piece.

\begin{tabular}{|c|c|c|c|c|c|c|}
\hline Music Piece & $\begin{array}{c}\text { Run } \\
\#\end{array}$ & $\begin{array}{c}\text { Buffer } \\
\#\end{array}$ & $\begin{array}{l}\mapsto \\
\%\end{array}$ & $\begin{array}{l}\overleftrightarrow{4} \\
\%\end{array}$ & $\sum_{\%}$ & $\begin{array}{l}\text { Valid } \\
>50 \%\end{array}$ \\
\hline Adagio & 1 & 20 & 83 & 0 & 83 & $\checkmark$ \\
\hline Adagio & 2 & 19 & 94 & 0 & 94 & $\checkmark$ \\
\hline Adagio & 3 & 21 & 93 & 0 & 93 & $\checkmark$ \\
\hline Golden Dreams & 1 & 30 & 93 & 0 & 93 & $\checkmark$ \\
\hline Golden Dreams & 2 & 30 & 83 & 0 & 83 & $\checkmark$ \\
\hline Golden Dreams & 3 & 29 & 93 & 0 & 93 & $\checkmark$ \\
\hline Rondo Alla Turca & 1 & 12 & 88 & 0 & 88 & $\checkmark$ \\
\hline Rondo Alla Turca & 2 & 12 & 93 & 0 & 93 & $\checkmark$ \\
\hline Rondo Alla Turca & 3 & 12 & 94 & 2 & 96 & $\checkmark$ \\
\hline Sonatina Op. 20 & 1 & 24 & 83 & 0 & 83 & $\checkmark$ \\
\hline Sonatina Op. 20 & 2 & 23 & 86 & 0 & 86 & $\checkmark$ \\
\hline Sonatina Op. 20 & 3 & 23 & 95 & 0 & 95 & $\checkmark$ \\
\hline Twenty-one Pilots & 1 & 14 & 93 & 0 & 93 & $\checkmark$ \\
\hline Twenty-one Pilots & 2 & 13 & 96 & 0 & 96 & $\checkmark$ \\
\hline Twenty-one Pilots & 3 & 14 & 88 & 0 & 88 & $\checkmark$ \\
\hline
\end{tabular}

Table 6.4: The necessary data for making decision for turning page at the middle of the each piece in online mode. 


\section{Chapter 7}

\section{Discussion}

This research attempts to decrease the complexity of previous studies in pageturning domain by utilizing wearable technologies instead of traditional video and audio-based processing methods. The two primary advantages of this alternative solution can be categorized into the following groups:

- Environmental factors such as noise, lights, and shadows, which have already been addressed as constraints, do not influence the new design.

- Conducting the experimental sessions and hardware installations can be arranged more responsive. Unlike past, the hardware components should not be fixed in a specific place, so the participants can freely move the wearable devices.

The proposed design consists of hardware (wearable device) and software parts. Like other automated systems that require precise synchronization of their components, the synchronization process relies entirely on the software 
part in this study. So, the wearable device merely has a passive role and continuously transmits its sensor data to the software part.

Observing the pianist's hand movements showed that by pressing piano keys, wrists move up and down slightly. So, the accelerometer sensor in wearable gadget — which are worn on the wrists - detects this movement and generates a significant spike in acceleration signal. The time sequences of pressed keys can be collected by tracking the trend of acceleration signal peaks.

In controlled conditions, if the pianists use equal pressure for playing each key, all peaks would get the same value. In this case, simple numerical search rules can identify all peaks. The data captured from experimental sessions showed that pianists frequently change their hand's pressure while playing, so signal peak values are utterly inconsistent. Additionally, for the parts that a series of notes should be played shortly after each other, the pianist's wrist usually does not move, and fingers press keys. Therefore, for that part, the first note only generates a high-value spike and recognizing other notes is difficult.

The frequency of accelerometer sensor was the first external factor that affected the number of peaks in the acceleration signal. At low frequencies (e.g., $50 \mathrm{~Hz}$ ), by pressing the piano keys gently, no significant spike appears in the acceleration signal. On the other hand, in higher frequencies (e.g., $200 \mathrm{~Hz}$ ), the sensitivity of the sensors increases, and a sudden wrist movement is reflected in signal completely. It should be noted that in high-frequency, the accelerometer generates some invalid local maximum points near the real peaks. Analyzing data revealed that the accelerometer sensor generates 
accurate spikes in high-frequency mode, so a heuristic function removed all invalid local maximums.

After applying various signal peak finding methods - that mostly focus on peak values, intervals, or thresholds - I found that the K-means function returns better clusters of peaks. So, by setting the $K=2$, all peaks with higher values were grouped into one cluster, and the rest peaks that had lower values were discarded. K-means approach is fully dynamic and can cope with a different style of playing.

Collecting digital notes from the MIDI file was not complicated. Many open-source libraries offer a toolbox for parsing MIDI files and extracting instruments and their notes. The time series of digital notes in this research refers to collecting the start time and duration of notes of each piece.

In general, converting MIDI files to readable music sheets is not a practical solution. For example, piano sheet music usually has two separated staves for the right and left hands, but some MIDI files merged those staves. The repeat bars, tempo, and dynamics are other limitations of using MIDI files as the music sheets. So, it is suggested to use music notation software to create music sheets and then export MIDI files for similar studies.

Undoubtedly, one of the challenging parts of this research was aligning time series - the time series of digital notes and time series of pressed keysto validate the pianist's performance. Dynamic time warping was the primary function that was used for finding the optimum alignment between series. In a best-case scenario that the pianist press all keys at the right time, all data points of both series should be aligned completely without any time lag. However, humans cannot control time like computers, so a short delay 
exists between aligning pairs. To overcome this lag, first, time series should be cut into smaller chunks. Next, a cross-correlation function finds the best correlation between aligned pairs in each chunk by adding a range of lags.

It is important to highlight that the combination of dynamic time warping, auto chunking, and cross-correlation helps when a music piece has various notes. For pieces like Asturias Leyenda by Isaac Albeniz, the first 24 measures only have the sixteenth notes. The auto chunking function can not cut the time series into smaller chunks; therefore, applying the cross-correlation function is also ineffective.

The music rhythm speed (tempo) was another critical factor in this study. Since the professional pianists did not participate in experimental sessions, the pieces with low $\mathrm{BMP}^{1}$ were selected. I realized that the accelerometer frequency for pieces with a higher tempo should be increased too. It is suggested to use music pieces with higher tempo (e.g., $120-140$ BMP) in future studies.

In my proposed design, any chunks can get the page-turning event. So, by reaching the nominated chunk, the decision model evaluates the type, status of previous chunks and distances of aligned pairs and decides to turn the digital sheet music.

The first validation step determines whether the pianist has performed the majority of notes before the page-turning point. Then the percentage of the one-to-one and aligned pairs of the previous chunk is measured. A rule is concluded by randomly placing the page-turning event in recorded data from experimental sessions and analyzing them; when the sum of one-to-one

\footnotetext{
${ }^{1}$ Beats per minute.
} 
and aligned pairs is more than 50\%, the pianist had performed reasonably, and the page-turning event can be executed.

In case the status and type of aligned pairs could not satisfy requirements, the system calculates the distance error of aligned pairs in the last attempts and use that as an evaluation metric.

In all experimental runs, the middle point of each piece was selected as the page-turning point. The previous chunk of all nominated points got the passed label. The sum of one-to-one and aligned pairs in all runs were more than $50 \%$, so the decision model successfully turned the digital music sheet.

The majority portion of alignment types in all experiments was one-toone $(90 \%)$. It can be concluded that the majority of notes in nominated chunks almost performed at the right time. 


\section{Chapter 8}

\section{Conclusions}

In this research, I proposed a framework that consists of hardware and software packages for making an automatic page-turner for pianists. Unlike previous studies in the score-following domain that mostly focused on video or sound processing methods, I used a pair of lightweight low-budget wearable devices. In proposed gadgets, the accelerometer sensor recognizes the pianist's wrist up and down movements while pressing piano keys. The custom software package finds the sequences of pressed keys from the acceleration signal and syncs them to digital music notes. This synchronization helps the system to understand which parts played by pianist. Eventually, a decision model evaluates synced data and decides whether to turn the digital music sheets at a specific time. All steps of this research were designed to answer the following questions. 
1. How can we track the pianist's wrists oscillatory motions (up and down) and track the sequences of these motions?

The proposed wearable gadgets have an inertial measurement unit with plastic housing and a body strap, so pianists can easily wear on their wrists. This kit has an accelerometer sensor that can sense and record motions in three-axes separately. By pressing piano keys, wrists move up and down slightly, and these movements generate notable spikes in the acceleration signal.

In this study, various functions such as noise reduction and data clustering were applied to the acceleration signal to prepare that for the peak detecting step. Some heuristic methods and K-means clustering function picked the acceleration signal peaks, and aggregating peaks returned the time series of pressed keys (TP).

2. How shall we synchronize the series of oscillatory motions with the sequences of digital notes, and determine the validity of the pianist performance to turn the music page at the right time? I selected MIDI file as a digital music format. MIDI file includes note properties like pitch, velocity, and note-on (the time that notes should be played). The collection of note-on values returned the timeseries of digital notes $(\mathrm{TN})$.

Finding the optimum alignment between the mentioned time-series was the biggest challenge in this study. Humans can not control time as accurately as computers, so I observed the time-lag resulting from rushing or dragging of playing notes throughout the performance. To overcome 
these problems, I suggested several approaches for dividing time-series into smaller chunks. Finally, the auto-chunking method solved the chunking problem, and the cross-correlation function reduced/removed the time latency between paired elements in both series.

The decision model is the last phase of the automatic page-turning solution. In this stage, the system decides whether to turn the digital sheet music, based on the heuristics validation model. The validation model is a complex mechanism that controls several factors such as the previous chunks alignment types, distance errors, and passing or failed labels.

I designed all stated steps for offline/tuning mode. In this mode, I analyzed data after each experiment. I also proposed the buffering mechanism as a solution for coping with real-time data. The result revealed that my heuristic solutions for signal processing and peak finding satisfied the first question. Moreover, other flexible and dynamic approaches made an accurate alignment between time-series.

The conclusion of results showed this system as a robust solution for automatic page-turner.

\subsection{Limitations}

The pandemic of COVID-19 indisputably impacted this research and most of the HCI studies in 2020. Since I played the role of participants in this study, I tried to change my playing style to simulate different users. I change the music pieces' tonality, played with different dynamics, and changed the 
standard fingering while playing. However, the advantages of having real participants should not be ignored.

\subsection{Future Studies}

The absence of real participants in this study was noted as a significant limitation, so conducting experimental research with real pianists to assess the presented framework is suggested for future study.

Learning to play the piano requires several years of practice and supervision, and it is better to identify and resolve any playing problems at the elementary levels. In this research, I used the accelerometer z-axis data only for detecting peaks, and the peaks value was not important. The force of pressing keys determines the value of peaks and represents the style of playing. Extra pressure could cause serious health issues specifically on wrist bones and should be considered in future works. 
Appendices 



\section{NGIMU Sensor Specifications}

- Sensors

- Triple-axis gyroscope $\left( \pm 2000^{\circ} / s, 400 \mathrm{~Hz}\right.$ sample rate $)$

- Triple-axis accelerometer $( \pm 16 g, 400 \mathrm{~Hz}$ sample rate)

- Triple-axis magnetometer $( \pm 1300 \mu T)$

- Barometric pressure (300-1100 hPa)

- Humidity sensor

- Temperature sensor

- Battery voltage, current, percentage, and time remaining

- Analogue inputs (8 channels, 0-3.1 V, 10-bit, $1 \mathrm{kHz}$ sample rate)

- Auxiliary serial (RS-232 compatible) for GPS or custom electronics/sensors

- Real-time clock

- Onboard data processing

- AHRS fusion algorithm provides a measurement of orientation relative to the Earth as a quaternion, rotation matrix, or Euler angles

- AHRS fusion algorithm provides a measurement of linear acceleration

- All measurements are timestamped

- Synchronisation of timestamps for all devices on a Wi-Fi network

- Communication interfaces

- USB

- Serial (RS-232 compatible)

- Wi-Fi (802.11n, $5 \mathrm{GHz}$, built-in or external antennae, AP or client mode)

- SD card (accessible as an external drive via USB)-

- Power management

- Power from USB, external supply, or battery

- Battery charging via USB or external supply

- Sleep timer

- Motion triggers wake up

- Wake up timer

- $3.3 \mathrm{~V}$ supply for user electronics (500 mA)

For more information check : https://x-io.co.uk/ngimu/ 


\title{
Python Code Sample
}

\section{pretty_midi}

\author{
import pretty_midi \\ path = "midi path" \\ pitch_collection, note_on_collection $=[],[]$ \\ midi $=$ pretty_midi.PrettyMIDI (path) \\ for index, note in enumerate(m.instruments[0].notes): \\ if (index $==0$ ): \\ note_on_collection.append (note.start) \\ pitch_collection. append (note.pitch) \\ else: \\ \# checking for music chords \\ if (note_on_collection [-1] != note.start): \\ note_on_collection.append (note.start) \\ pitch_collection.append (note.pitch)
}

\section{fastdtw}

from scipy.spatial.distance import euclidean

from fastdtw import fastdtw

observed $=[1,2,4,6,7,8,9,10,14,18,19,20]$

predicted $=[0,2,4,7,7,7,9,11,12,17,18]$

distance, path $=$ fastdtw(observed, predicted, dist=euclidean)

$\gg$ distance $=10.00$

$\gg$ path $=[(0,0),(1,1),(2,2),(3,3),(4,4),(5,5),(6,6),(7,7),(8,8),(9,9),(10,10),(11,10)]$

\section{kmeans1d}

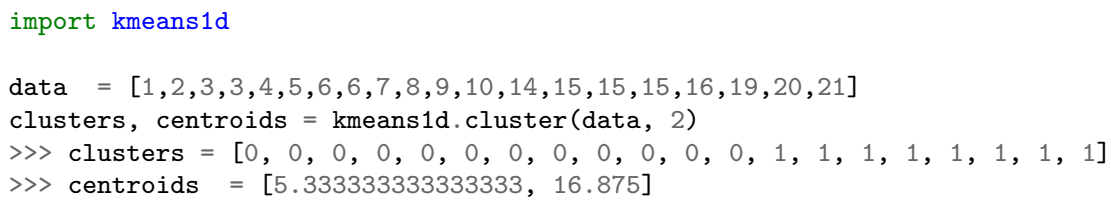

\section{sklearn}

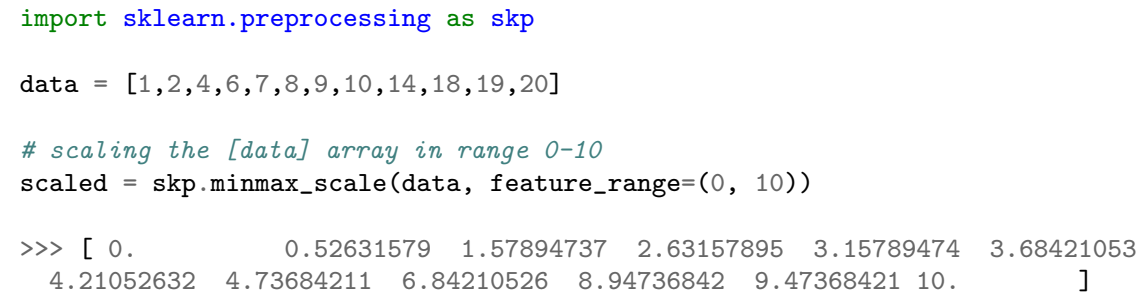




\section{Bibliography}

[1] George Wolberg and Irene Schipper. Page turning solutions for musicians: A survey. Work, 41(1):37-52, 2012.

[2] Joseph H Machnacz. Page turner for sheet music and the like, May 23 1972. US Patent 3,665,093.

[3] Andreas Arzt, Gerhard Widmer, and Simon Dixon. Automatic page turning for musicians via real-time machine listening. In ECAI, pages 241-245, 2008.

[4] Andreas Arzt, Sebastian Böck, Harald Flossmann, Sebastian aFrostel, Martin Gasser, Cynthia CS Liem, and Gerhard Widmer. The piano music companion. In ECAI, pages 1221-1222, 2014.

[5] André Tabone. Automated page turner for musicians. B.S. thesis, University of Malta, 2019.

[6] Qiongfeng Shi, Bowei Dong, Tianyiyi He, Jianxiong Sun, Zhongand Zhu, Zixuan Zhang, and Chengkuo Lee. Progress in wearable electronics/photonics - moving toward the eof artificial intelligence and internet of things. InfoMat, 2020.

[7] Hanlu Ye, Meethu Malu, Uran Oh, and Leah Findlater. Current and future mobile and wearable device use by people wivisual impairments. In Proceedings of the SIGCHI Conference on Human Factors Computing Systems, pages 3123-3132, 2014.

[8] Jing Wu, He Li, Zhangxi Lin, and Haichao Zheng. Competition in wearable device market: the effect of netwoexternality and product compatibility. Electronic Commerce Research, 17(3):335-359, 2017.

[9] Seyed Ali Mirazimzadeh and Victoria McArthur. Automatic page-turner for pianists with wearabmotion detector. In International Conference on Human-ComputInteraction, pages 209-218. Springer, 2020. 
[10] Wikipedia. Musical notation - Wikipedia, the free encyclopedia. https://w.wiki/PFq, 2020. [Online; accessed 26-April-2020].

[11] Wikipedia. List of musical symbols - Wikipedia, the free encyclopedia. https://w.wiki/PFp, 2020. [Online; accessed 27-April-2020].

[12] Cory McKay. Automatic genre classification of MIDI recordings. $\mathrm{PhD}$ thesis, McGill University Canada, 2004.

[13] Free music composition and notation software - musescore. https: //musescore.org/en. (Accessed on 06/05/2020).

[14] Alexandra Maria Aluţei, Beniamin Vasile Chetran, Ion Lungu, and Dan Mândru. Automatic page turner. In Solid State Phenomena, volume 166, pages 27-32. Trans Tech Publ, 2010.

[15] Ellaine Abueg, John Coughlin, N Hanumara, O Koenig, A Tillinghast, and $\mathrm{T}$ Malaghan. Implementation of a re-usable adhesive in a novel automated page-turner. In IEEE 30th Annual Northeast Bioengineering Conference, 2004. Proceedings of the, pages 208-209. IEEE, 2004.

[16] Satoko Kora, Billy BL Lim, and James Wolf. A hands-free music score turner using google glass. Journal of Computer Information Systems, 59(4):297-307, 2019.

[17] Douglas J Carr and Lincoln Lucero. Portable page turner for music sheets, April 20 1993. US Patent 5,203,248.

[18] Lorin Grubb and Roger B Dannenberg. A stochastic method of tracking a vocal performer. In $I C M C, 1997$.

[19] Roger B Dannenberg. An on-line algorithm for real-time accompaniment. In $I C M C$, volume 84, pages 193-198, 1984.

[20] Barry Vercoe. The synthetic performer in the context of live performance. In Proceedings of International Computer Music Conference, pages 199-200, 1984.

[21] Francisco Jose Rodriguez-Serrano, Julio Jose Carabias-Orti, Pedro VeraCandeas, and Damian Martinez-Munoz. Tempo driven audio-to-score alignment using spectral decomposition and online dynamic time warping. ACM Transactions on Intelligent Systems and Technology (TIST), 8(2):1-20, 2016. 
[22] Wataru Inoue and Shuji Hashimoto. Adaptive karaoke system: Human singing accompaniment based on speech recognition. In Proceedings of the International Computer Music Conference, pages 70-70. INTERNATIONAL COMPUTER MUSIC ACCOCIATION, 1994.

[23] Wataru Inoue. A computer music system for human singing. In Proc. Int. Computer Music Conf., pages 150-153, 1993.

[24] Roger B Dannenberg and Ning Hu. Polyphonic audio matching for score following and intelligent audeditors. 2003.

[25] Nicola Orio and Diemo Schwarz. Alignment of monophonic and polyphonic music to a score. 2001.

[26] César Alonso Abad. Pitch-synchronous multiresolution analysis of music signals. PhD thesis, Citeseer, 2007.

[27] Arshia Cont. A coupled duration-focused architecture for real-time music-to-score alignment. IEEE transactions on pattern analysis and machine intelligence, 32(6):974-987, 2009.

[28] Nicola Orio, Serge Lemouton, and Diemo Schwarz. Score following: State of the art and new developments. 2003.

[29] G. Bradski. The OpenCV Library. Dr. Dobb's Journal of Software Tools, 2000.

[30] Martín Abadi, Paul Barham, Jianmin Chen, Zhifeng Chen, Andy Davis, Jeffrey Dean, Matthieu Devin, GhemawaSanjay, Geoffrey Irving, Michael Isard, et al. Tensorflow: A system for large-scale machine learning. In 12th \{USENIX\} Symposium on Operating Systems Design and Implementation (\{OSDI\} 16), pages 265-283, 2016.

[31] NVIDIA, Péter Vingelmann, and Frank H.P. Fitzek. Cuda, release: 10.2.89, 2020.

[32] Dmitry O Gorodnichy and Arjun Yogeswaran. Detection and tracking of pianist hands and fingers. In The 3rd Canadian Conference on Computer and Robot Vision (CRV'06), pages 63-63. IEEE, 2006.

[33] Potcharapol Suteparuk. Detection of piano keys pressed in video. Dept. of Comput. Sci., Stanford Univ., Stanford, CA, USA, Tech. Rep, 2014. 
[34] David Johnson, Isabelle Dufour, Daniela Damian, and George Tzanetakis. Detecting pianist hand posture mistakes for virtual piano tutoring. ICMC 2016 - 42nd International Computer Music Conference, Proceedings, pages 167-170, 2016.

[35] Yoshinari Takegawa, Tsutomu Terada, and Shojiro Nishio. Design and implementation of a real-time fingering detection system for piano performance. International Computer Music Conference, ICMC 2006, 74(Icmc):67-74, 2006.

[36] Akiya Oka and Manabu Hashimoto. Marker-less piano fingering recognition using sequential depth images. FCV 2013 - Proceedings of the 19th Korea-Japan Joint Workshop on Frontiers of Computer Vision, 4:1-4, 2013.

[37] Zhenyu Tang, Chenyu Yan, Sijie Ren, and Huagen Wan. Headpager: Page turning with computer vision based head interaction. In Asian Conference on Computer Vision, pages 249-257. Springer, 2016.

[38] Frédéric Bevilacqua, Florence Baschet, and Serge Lemouton. The augmented string quartet: experiments and gesture following. Journal of New Music Research, 41(1):103-119, 2012.

[39] Thomas J Mitchell, Sebastian Madgwick, and Imogen Heap. Musical interaction with hand posture and orientation: A toolbox of gestural controlmechanisms. 2012.

[40] Simon Dixon. An on-line time warping algorithm for tracking musicperformances. In IJCAI, pages 1727-1728, 2005.

[41] Wenjun Lu, Avinash Varna, and Min Wu. Secure video processing: Problems and challenges. In 2011 IEEE International Conference on Acoustics, Speech and SignProcessing (ICASSP), pages 5856-5859. IEEE, 2011.

[42] Deyan Chen and Hong Zhao. Data security and privacy protection issues in cloud computing. In 2012 International Conference on Computer Science and ElectroniEngineering, volume 1, pages 647-651. IEEE, 2012.

[43] Norah Almohaimeed and Master Prince. A comparative study of different oject tracking methods in a video. International Journal of Computer Applications, 975:8887. 
[44] Hao Jiang and Mark S Drew. Tracking objects with shadows. In Image and Video Communications and Processing 2003, volume 5022, pages 512-521. International Society for Optics and Photonics, 2003.

[45] Alexandra Bonnici, Stefania Cristina, and Kenneth P Camilleri. Preparation of music scores to enable hands-free page turning based on eyegaze tracking. In Proceedings of the 2017 ACM Symposium on Document Engineering, pages 201-210, 2017.

[46] imu - past and present — international mathematical union.

[47] Oliver J Woodman. An introduction to inertial navigation. Technical report, University of Cambridge, Computer Laboratory, 2007.

[48] Ngimu - x-io technologies. https://x-io.co.uk/ngimu/. (Accessed on 05/12/2020).

[49] Guido Van Rossum and Fred L Drake Jr. Python reference manual. Centrum voor Wiskunde en InformatiAmsterdam, 1995.

[50] Cory S Myers. A comparative study of several dynamic time warping algorithms for speech recognition. PhD thesis, Massachusetts Institute of Technology, 1980.

[51] Ning Hu, Roger B Dannenberg, and George Tzanetakis. Polyphonic audio matching and alignment for music retrieval. In 2003 IEEE Workshop on Applications of Signal Processing to Audio aAcoustics (IEEE Cat. No. 03TH8684), pages 185-188. IEEE, 2003.

[52] Hesam Izakian, Witold Pedrycz, and Iqbal Jamal. Fuzzy clustering of time series data using dynamic time warping distance. Engineering Applications of Artificial Intelligence, 39:235-244, 2015.

[53] François Petitjean, Alain Ketterlin, and Pierre Gançarski. A global averaging method for dynamic time warping, with applications to clustering. Pattern Recognition, 44(3):678-693, 2011.

[54] Guillaume Plouffe and Ana-Maria Cretu. Static and dynamic hand gesture recognition in depth data using dynamic time warping. IEEE transactions on instrumentation and measurement, 65(2):305-316, 2015.

[55] Gineke A Ten Holt, Marcel JT Reinders, and Emile A Hendriks. Multidimensional dynamic time warping for gesture recognition. In Thirteenth annual conference of the Advanced School for Computing and Imaging, volume 300, page 1, 2007. 
[56] Ahmad Akl and Shahrokh Valaee. Accelerometer-based gesture recognition via dynamic-time warping, affinity propagation, \& compressive sensing. In 2010 IEEE International Conference on Acoustics, Speech and Signal Processing, pages 2270-2273. IEEE, 2010.

[57] Andrea Corradini. Dynamic time warping for off-line recognition of a small gesture vocabulary. In Proceedings IEEE ICCV workshop on recognition, analysis, and tracking of faces and gestures in real-time systems, pages 82-89. IEEE, 2001.

[58] Po-Wen Chou, Fu-Neng Lin, Keh-Ning Chang, and Herng-Yow Chen. A simple score following system for music ensembles using chroma and dynamic time warping. In Proceedings of the 2018 ACM on International Conference on Multimedia Retrieval, pages 529-532, 2018.

[59] Andreas Arzt. Score following with dynamic time warping; an automatic page-turner. PhD thesis, 2008.

[60] AL Jacobson. Auto-threshold peak detection physiological signals. In 2001 Conference Proceedinof the 23rd Annual InternationConference of the IEEE Engineering Medicine and Biology Society, volume 3, pages 2194-2195. IEEE, 2001.

[61] Andrew Wee, Yonggang Grayden, Davidand Zhu, PetkovicDuraKarolina, and David Smith. A continuous wavelet transfoalgorithm for peak detection. Electrophoresis, 29(20):4215-4225, 2008.

[62] Vaggelis P Tzallas, Alexandros T aOikonomou and FotiadiDimitrios I. Epileptic spike detection usingkalman filter based approach. In 2006 International Conferenof the IEEE Engineering in Medicine aBiology Society, pages 501-504. IEEE, 2006.

[63] Douglas A Coast, SterRichard M, and Stanley A Cano, Gerald $\mathrm{G}$ aBriller. An approach to cardiac arrhythmanalysis using hidden markov models. IEEE Transactions on biomedicEngineering, 37(9):826836, 1990.

[64] Tianfeng Chai and Roland R Draxler. Root mean square error (rmse) or mean absoluerror (mae)?--arguments against avoiding rmse in tliterature. Geoscientific model development, 7(3):1247-1250, 2014.

[65] Cort J Willmott and Kenji Matsuura. Advantages of the mean absolute error (maover the root mean square error (rmse) in assessiaverage model performance. Climate research, 30(1):79-82, 2005. 